\title{
Advances in computational intelligence of polymer composite materials: Machine learning assisted modeling, analysis and design
}

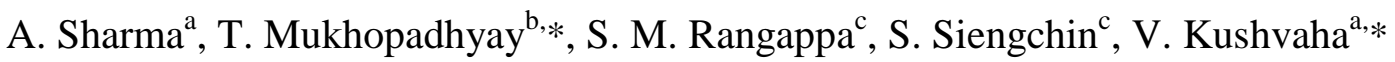 \\ ${ }^{a}$ Department of Civil Engineering, Indian Institute of Technology Jammu, J\&K, India \\ ${ }^{b}$ Department of Aerospace Engineering, Indian Institute of Technology Kanpur, Kanpur, India \\ ${ }^{c}$ Natural Composites Research Group Lab, King Mongkut's University of Technology North Bangkok \\ *Email address: vinod.kushvaha@iitjammu.ac.in (V.K.), tanmoy@iitk.ac.in (T.M.)
}

\begin{abstract}
The superior multi-functional properties of polymer composites have made them an ideal choice for aerospace, automobile, marine, civil, and many other technologically demanding industries. The increasing demand of these composites calls for an extensive investigation of their physical, chemical and mechanical behavior under different exposure conditions. Machine learning (ML) has been recognized as a powerful predictive tool for data-driven multi-physical modeling, leading to unprecedented insights and exploration of the system properties beyond the capability of traditional computational and experimental analyses. Here we aim to abridge the findings of the large volume of relevant literature and highlight the broad spectrum potential of ML in applications like prediction, optimization, feature identification, uncertainty quantification, reliability and sensitivity analysis along with the framework of different ML algorithms concerning polymer composites. Challenges like the curse of dimensionality, overfitting, noise and mixed variable problems are discussed, including the latest advancements in ML that have the potential to be integrated in the field of polymer composites. Based on the extensive literature survey, a few recommendations on the exploitation of various ML algorithms for addressing different critical problems concerning polymer composites are provided along with insightful perspectives on the potential directions of future research.
\end{abstract}

Keywords: Machine learning in polymer composites; Prediction and characterization; Optimization; Uncertainty quantification; Curse of dimensionality; AI and ML in polymer science 


\section{Table of contents}

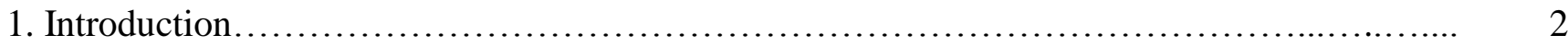

2. Multifaceted applications of machine learning in polymer composite...................... 8

2.1. Basic framework of machine learning paradigm in polymer composites................ 10

2.2. Prediction, optimization and uncertainty quantification............................. 13

2.3. Reliability and sensitivity analysis .......................................... 20

3. Sampling techniques for training machine learning models.......................... 21

4. Widely adopted machine learning algorithms in polymer composites...................... 23

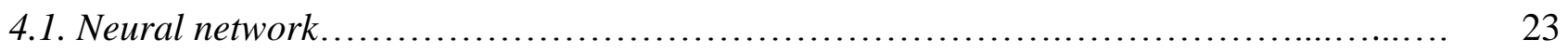

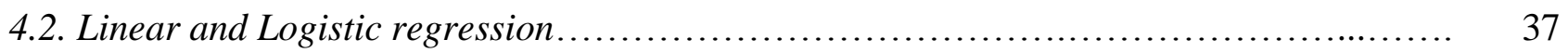

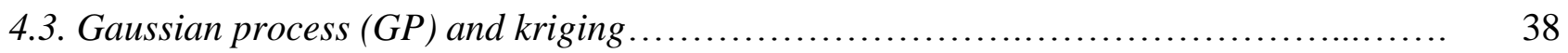

4.4. Support vector machines $(S V M)$.......................................... 41

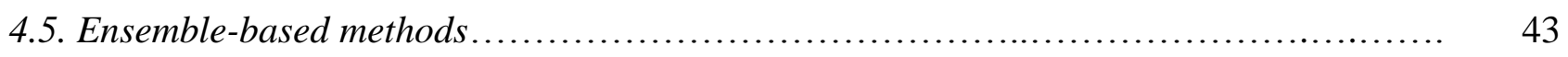

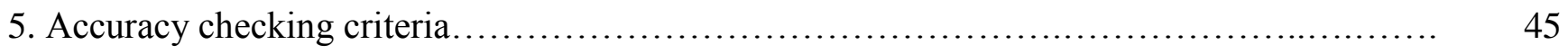

6. Summary and perspective................................................... 47

6.1. Curse of dimensionality ..................................................... 48

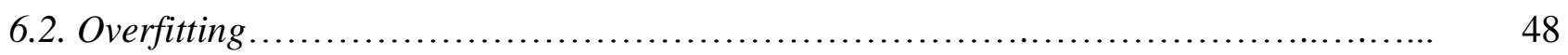

6.3. Mixed variable problems................................................... 49

6.4. Robustness under the influence of noise ...................................... 50

6.5. Latest trends and future road maps......................................... 51

7. Conclusions............................................................ 53

Acknowledgments.......................................................... 54

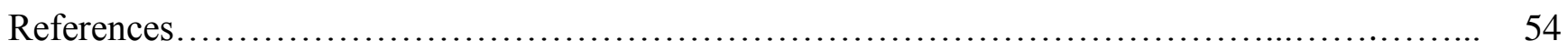

\section{Introduction}

Aerospace, automobile, marine, civil and many other technologically demanding industries are looking for alternative multi-functional materials which have a combination of excellent tunable properties like high strength, light-weight, corrosion resistance, thermal insulation, acoustic damping, high fracture toughness along with aesthetic features. Polymer composites are found to have a good mix of the above-mentioned properties along with rapid and easy manufacturability which makes them a suitable alternative material to be utilized in various modern industries [1-5]. Generally, polymer 
composite is made up of two phases: the matrix phase (continuous) and the reinforcement phase (dispersed). Usually, a thermosetting or thermoplastic organic polymer serves as the matrix, the basic purpose of which is to bind the reinforcement and transfer the load uniformly to the embedded reinforcement [6]. Different types of materials are used to strengthen the polymeric matrix and are known as reinforcing agents. These materials can be in the form of natural or man-made fibers, particles, whiskers and fragments [7,8]. Some examples include glass, carbon, kevlar, aramid, flax, hemp, jute, sisal, coir, alumina, mica, basalt etc. [9]. Considering the environmental and sustainability issues, biocomposites are getting popular recently and for this purpose biofibers and biopolymers are being used [10]. Most of the biofibers and biopolymers are biodegradable in nature and are a great solution for the waste disposal problem. The most important advantage of using the biopolymers as matrix in composites is that they are renewable and neutral in terms of carbon. However, the challenge in ramping up the applicability of such composites is the huge variation in their properties as they get affected by a number of variables like the type of biopolymer and the natural fibers used, environmental condition of their source, type of the fiber modification and the processing techniques [11]. From a computational viewpoint, this leads to a significantly large space of input dimensions, which makes the analysis and design process more complicated. On a larger perspective, polymer composites are classified into different categories based on the type of reinforcing material used (refer to Figure 1).

Shape, size, chemical composition, amount of the reinforcement and the way reinforcing agents are added along with the manufacturing process of the composite greatly affect the overall resulting properties of the composite materials [12-18]. If continuous fibers are used to reinforce the matrix, then parameters like orientation of the fibers and the stacking sequence are important parameters to be considered. If the composite is particulate reinforced, then the aspect ratio of the reinforcing particles plays an important role [19,20]. The next significant aspect that has a considerable impact on the mechanical properties of the polymer composites is the interface between the two phases of the composite [21]. An interface is basically a two dimensional zone between two layers having different microstructure [22]. Some specific physical and chemical interactions that occur at the interface results in the formation of a three dimensional zone called interphase [23]. In the context of polymer composites, noticeable alterations in the mobility of the polymeric chain, crystallinity, the extent of curing and the microstructure take place in the region of interphase [24,25]. Thus the perturbations caused at the interphase due to the presence of reinforcement in the long chains of polymer matrix result in a significant impact on the properties of the resulting composite. The extent of these perturbations depends on the size and the volume fraction of the fillers (reinforcement) used [20,21]. 


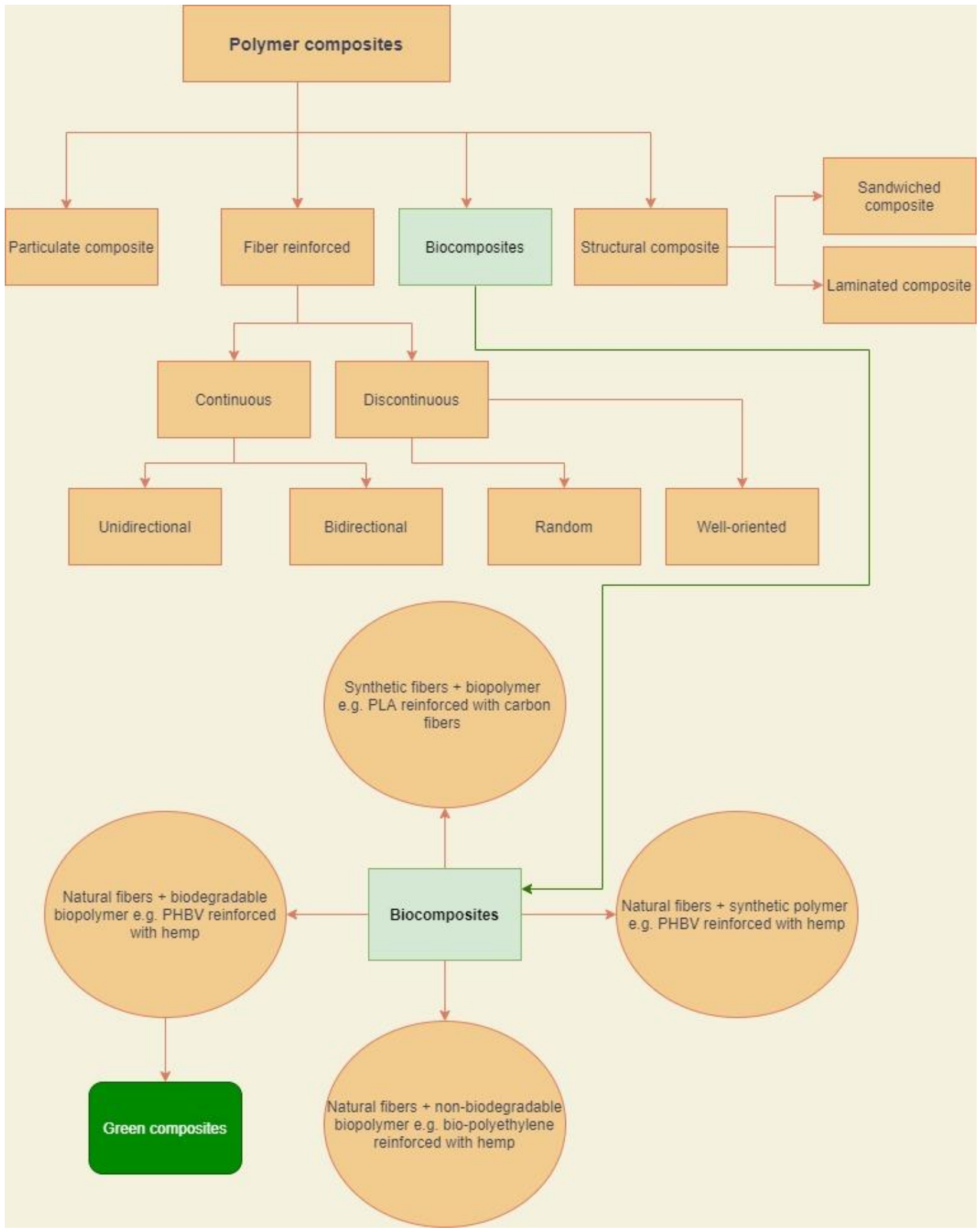

Figure 1: Classification of polymer composites. This figure shows different types of matrix and reinforcing materials that can be used in polymer composites. As typical illustrative examples, we further explain the classifications of fiber-reinforced composites, structural composites and biocomposites in the figure. The particulate composites could have different uniform and non-uniform shapes of the particles which are embedded in the matrix. 
The chemical composition of the polymer matrix and the reinforcement, along with the filler geometry affect the overall chemistry and bring in significant changes in the interfacial zone and the global morphology [15,28-30]. Toughening mechanism, cavitation (the extent of plastic yielding), fiber bridging, fatigue behavior and the shear strength of the overall composite largely get affected by the kind of interphase developed [31-33]. Therefore, it is important to possess a good understanding about the nature of interfacial region while designing composite materials in order to suit a specific application. The process used for manufacturing the composite material is also a governing factor in deciding the mechanical response along with the long term performance of the resulting composite. Hand lay-up method, compression molding, resin transfer molding, injection molding, direct extrusion, pultrusion and vacuum molding are the processes generally used to manufacture composite materials. The choice of manufacturing process depends on the intended application of the final product because each of the mentioned processes possesses different processing speed, extent of void formation and curing temperature [34-36]. Inappropriate choice of manufacturing technique may lead to very high shear stress at the boundary of the reinforcement. Hence these key parameters act as process variables and need to be optimized considering the final application [37]. Another influencing parameter on the mechanical behavior of composite materials is the residual stress and strain [38,39]. Stress transfer from the continuous phase to the dispersed phase is a very important phenomenon that critically affects the strength and stiffness of the composites [40]. The difference in the elastic modulus and the Poisson's ratio has a vital role to play in the mechanism of stress transfer [41]. Coefficient of thermal expansion of the matrix and the reinforcement is also needed to be taken into account since a mismatch in this coefficient may lead to the development of thermal residual stresses [42].

For developing composites of higher specific strength and stiffness along with higher impact, wear and fatigue resistance, it is important to consider a compound effect of the parameters mentioned above. Polymer composites thus have the advantage of synergistic properties which can easily be tailored for achieving a desirable specific set of properties by selecting the appropriate combination of continuous and dispersed phase [43]. Flexible and economical materials suiting a wide range of simultaneous objectives are what most modern industries demand. To explore the broader efficacy of polymer composites, it is important not to limit the composites to only one type of demand or objective by considering only a few process parameters while keeping others as constant. Rather all the process parameters are required to be considered simultaneously for optimization and that would be a remarkable contribution in the field of material design. Modeling the complex relationships between the governing parameters (both input and output) is extremely strenuous. Despite the availability of large experimental setups and computational tools, it is laborious and time-consuming to investigate the 
significance of each of the governing parameters experimentally. Over the last two decades, material science has undergone a steady shift from the phase of developing purely computational techniques for the discovery and design of new and complex materials to the phase of developing coupled methods that increase the reliability of results by making use of computational predictions and experimental validation. Finite element and molecular dynamics simulation methods have been used to model the material behavior in various fields, but the complexity and computational intensiveness of the methods have encouraged the research society to look for other alternatives [44-49]. Therefore, many researchers have relied on the machine learning approach to determine the significance of the process parameters for an optimal design [50,51]. Machine learning (ML) provides a wider scope for efficiently investigating the behavior of resulting composites with limited experimentation or computationally intensive realizations of expensive models (refer to Figure 2). Exploitation of ML allows to achieve various tunable multifunctional properties in polymer composites simultaneously based on the constituent components and their arrangements or proportions. There are various machine learning algorithms that are being utilized in material science depending upon the type of problem and the dataset available. Though various studies summarize how the ML algorithms can be used in material science, most of the studies are limited in scope by the constraints of multiple variables which result in increased dimensionality and uncertainty caused by the randomness of the data. This review paper aims to abridge the findings of the large library of studies to highlight the broad spectrum potential of ML in polymer composites along with providing a roadmap for dealing with the critical issues.

In the last few years, machine learning has been recognized as a powerful tool used for efficient predictive modeling in the field of polymer composites that could lead to unprecedented insights and exploration of the system properties beyond the capability of traditional computational and experimental analyses. The fascinating abilities of ML have attracted a great deal of interest of the materials scientists and engineers in the field of polymer composites and subsequently, a significant number of research papers have been published dealing with various critical issues. In this article, a comprehensive overview of the applications of ML like prediction, optimization, feature identification, uncertainty quantification, reliability and sensitivity analysis would be presented along with the framework of different machine learning algorithms used in the field of polymer composites. Challenges like the curse of dimensionality, overfitting, noise and mixed variable problems would be discussed. Based on extensive literature survey, a few recommendations on the usage of various ML algorithms for addressing the critical problems concerning polymer composites would be provided with insightful perspectives on the evolving future research directions. Hereafter, the paper is organized as, section 2: a general overview of multifaceted applications of ML in the field of polymer composites, 


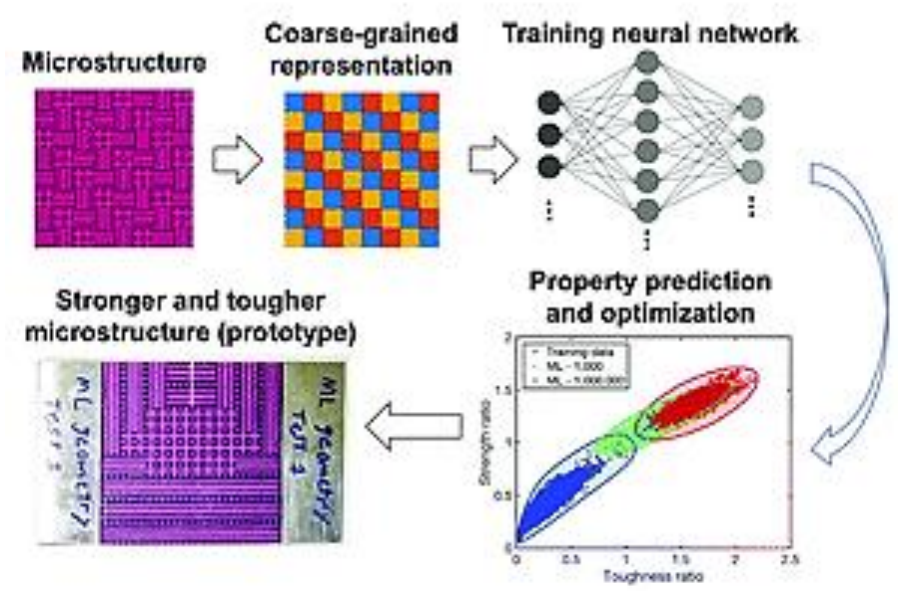

(a)

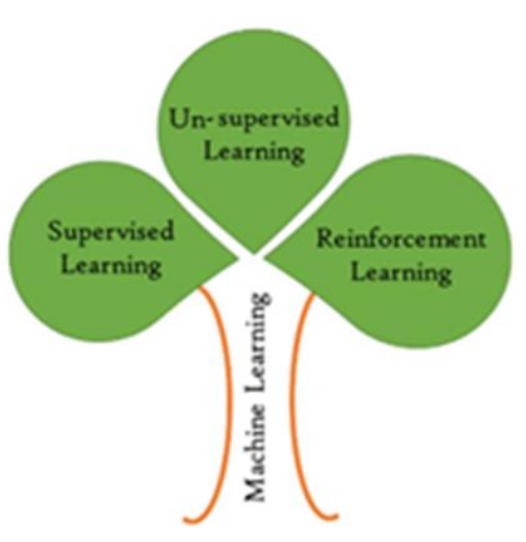

(b)

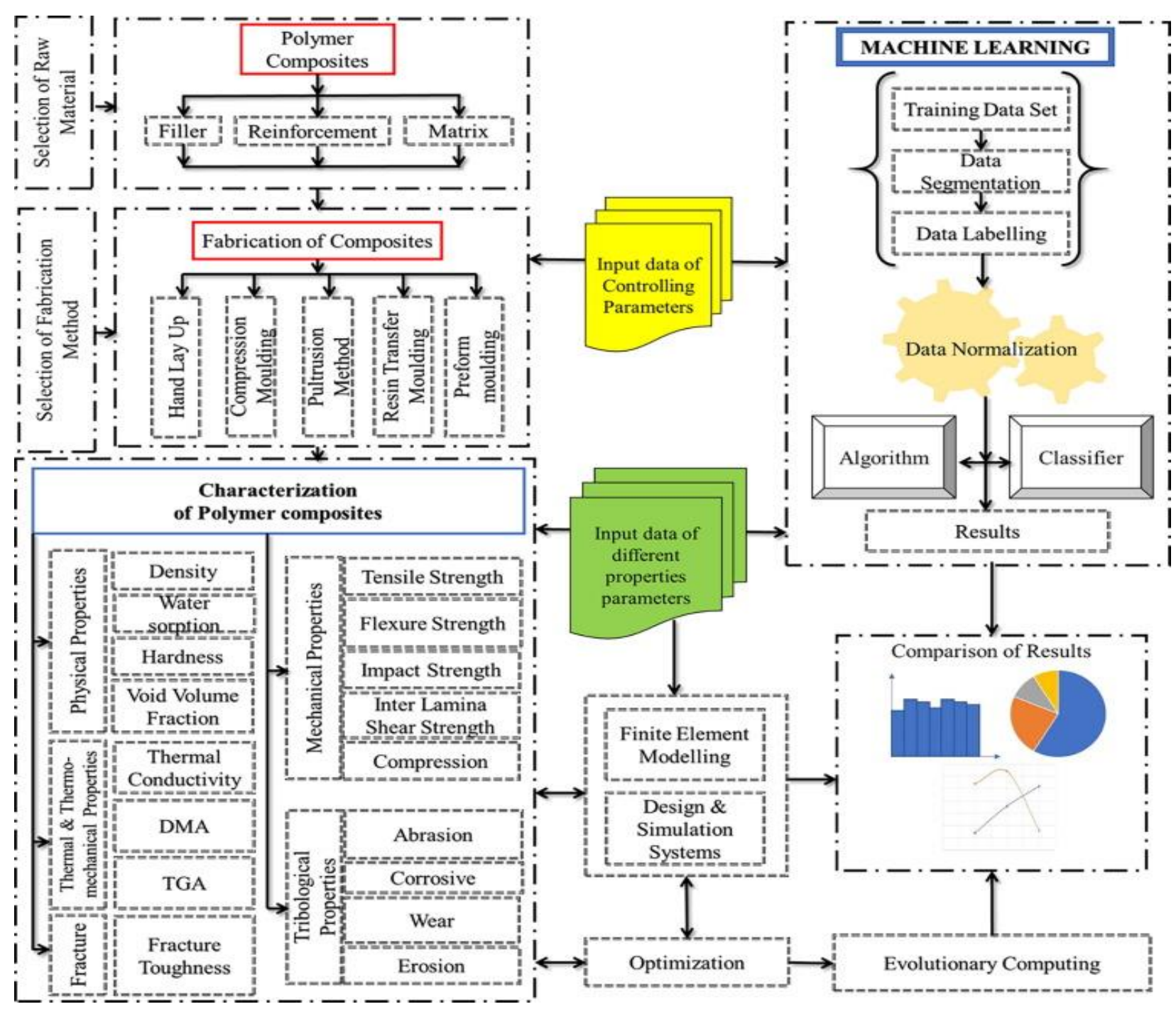

(c)

Figure 2: Typical examples and algorithms for ML assisted prediction in polymer composites. (a) Property prediction and optimization of polymer composites using convolutional neural network [52] (b) Predominant types of ML algorithms used in polymer composites (c) Application of machine learning for predicting the overall behavior of fiber-reinforced polymer composites [53]. 
section 3: critical review of the sampling techniques used for ML model formation, section 4: brief descriptions and review of commonly adopted ML algorithms, section 5: different approaches for ensuring accuracy and quality of ML models, section 6: discussions on a few critical issues encountered in ML based analyses of polymer composites and an overview of future research directions, section 7: concluding remarks.

\section{Multifaceted applications of machine learning in polymer composites}

A brain-cell interaction model was created by Donald Hebb in 1949. He mentioned it in a book entitled, 'The organization of behavior' [54], wherein he presented the brain theory of neurons. The theory presented in this book is considered to be the first stepping-stone in the development of machine learning concepts. The first notion of Machine Learning (ML) came in 1959 through Arthur Samuael who defined it as a field of study that gives computers the ability to learn without being explicitly programmed. It drew attention of many researchers and they started investigating earnestly in this area. After the 1990's ML caught its pace with the growing research and started being used in various fields like data analytics, predictive analysis, self-driving cars, fraud detection and prevention, stock exchange, text generation and analysis, image and face recognition and pattern recognition [55-58]. Gradually machine learning became a very exciting tool in the overall research community wherein various statistical and probabilistic methods were proven to accelerate the fundamental and applied research [59]. Machine learning techniques have a long history of application in the fields of biology and chemistry [60-62]. The huge success of ML algorithms in these fields encouraged material scientists to explore the possibility of utilizing the same in designing and developing new materials having superior properties and wider applications [63].

Conventionally experimentation used to play the key role in discovering and characterizing novel materials. But the advent of computational approaches has revolutionized the field of material science and the combination of experiments and simulations has proven to be an efficient way of exploring new possibilities $[64,65]$. In the analysis of polymer composites, a large number of existing parameters and various combinations which are needed to be evaluated in order to upsurge the applications with multi-functional demands, researchers are motivated to conduct more systematic and data-intensive research. The combination of experiments and computer simulations has been producing a huge expanse of data which has made it possible to integrate machine learning algorithms with material science. Many successful attempts can be found in the literature which are reviewed and summarized in this paper for bringing to light the wider acceptability along with promoting the usage of this reliable and very powerful tool further. Figure 3 is an illustration of the numerous applications 


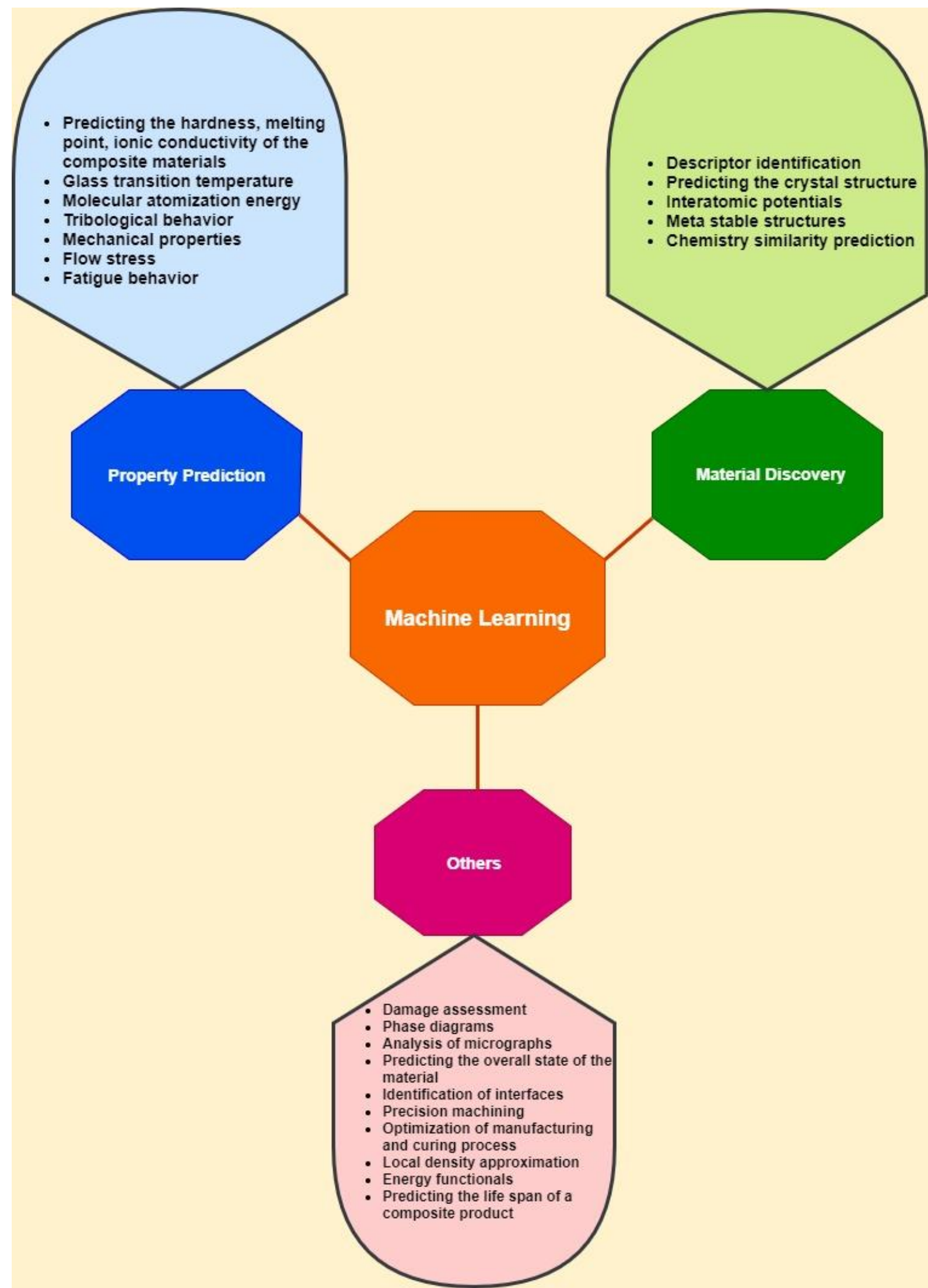

Figure 3: Applications of ML in various fields of polymer composites and material science. This figure shows the two major applications of machine learning as property prediction (/ characterization) and novel material discovery. Other evolving areas are also duly mentioned. 


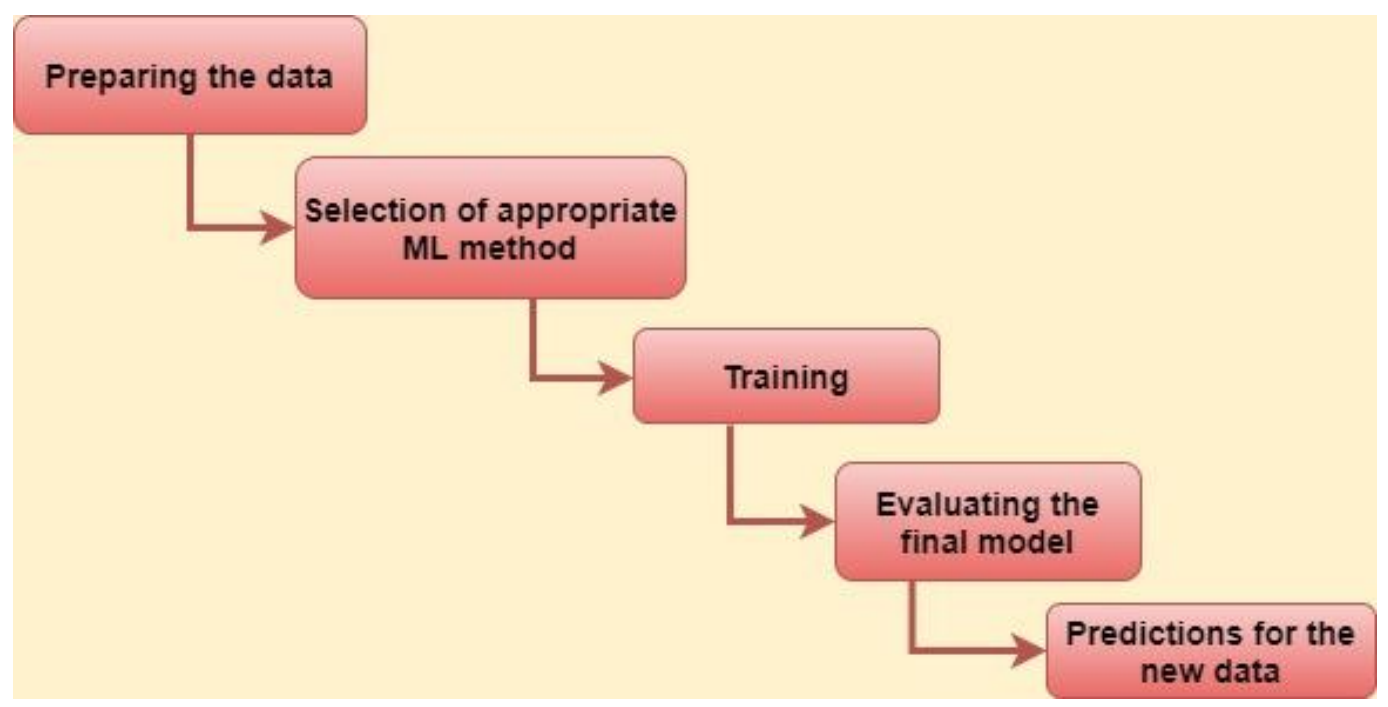

Figure 4: Basic workflow of ML algorithms. The majority of machine learning techniques that fall under the category of supervised learning follow a five-stage approach as described in the figure. Here any machine learning algorithm can be adopted depending on the efficiency and suitability for a particular type of problem (refer to section 4).

of machine learning in polymer composites and material science, encompassing property prediction (/ characterization), novel material discovery and other evolving areas. In the following subsections, we briefly discuss the vast scope for applicability of ML along with the respective algorithms focusing on the literature concerning polymer composites.

\subsection{Basic framework of machine learning paradigm in polymer composites}

Supervised and unsupervised learning are two widely accepted machine learning paradigms which are used in material science. In supervised learning, a well labeled dataset is generated and input parameters are mapped with the known outputs based on which predictions for the new data are made $[66,67]$. While in unsupervised learning, problem in hand is approached without much information about the output. It allows the user to discover the patterns in the data by deriving a structure in the dataset which is based on clustering and associations rules [68,69]. In case of polymer composites, supervised learning is more commonly used. Figure 4 shows the basic workflow of applying supervised learning techniques to predict the material behavior.

Data preparation is the first most critical and imperative step in utilizing any of the ML techniques [70]. One subset of material science is material informatics which is focused on modifying the form of data so as to utilize the available information effectively and this is where most of the effort of ML scientists goes in. Data acquisition in a systematic way is very important so that all the relevant explanatory variables are considered. After collecting the appropriate data, preprocessing is done in 


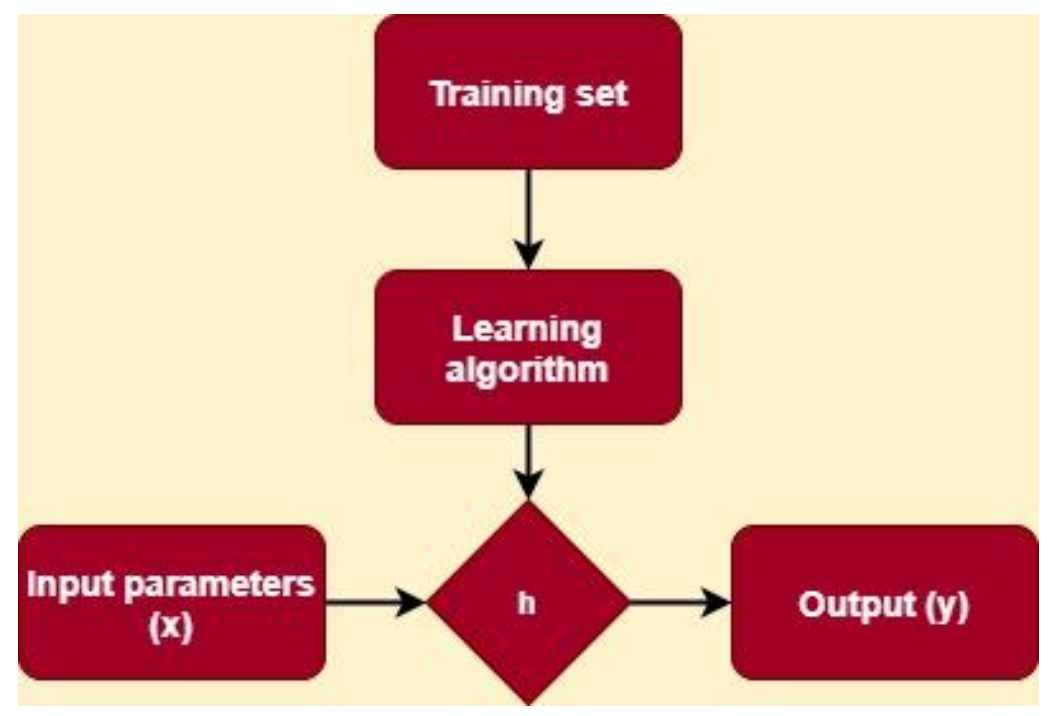

Figure 5: Schematic representation of ML algorithms. The figure illustrates the basic scheme for initial implementation of any learning algorithm $(\mathbf{h}: \mathbf{x} \rightarrow \mathbf{y})$ where, $\mathbf{h}$ represents a hypothesis function that maps the input parameters (x) to the output (y) and selects a suitable learning algorithm to be used for further prediction.

terms of formatting, cleaning and then sampling it. Formatting helps in bringing a structure in the data which ultimately enhances the data quality. Some of the attributes are deleted in the cleaning step so as to keep only the relevant parameters and sampling is used to select a subset of the data out of a big chunk which can further be utilized for the training purpose in machine learning [71,72]. Converting the raw information into certain relevant attributes which are further used as input features for the selected algorithm is a necessary step for getting accurate predictions and is commonly known as feature engineering [73]. It helps in increasing the learning accuracy along with improved comprehensibility [74-76].

After preparing the data well, the next step is to set a hypothesis function $(h(x))$ which maps the input parameters $(x)$ to the output $(y)$ and select a suitable learning algorithm to be used. Based on what kind of data we have and whether the problem in hand is classification or regression, an appropriate machine learning algorithm is chosen [77]. For a classification problem, mostly used algorithms include K-Nearest Neighbor (KNN), decision trees, neural networks, naive bayes and support vector machine [78]. If the problem is regression, then algorithms like linear regression, support vector regression, neural networks, Gaussian process and ensemble methods are used [79].

The next step is to train the chosen model with the processed data. The available data is split into three subsections namely training, cross-validation and testing dataset. The model learns to process the information using a training dataset. Cross-validation dataset is used for parameter tuning and to avoid the problem of overfitting. Evaluating the model is an essential part of the model 


\begin{tabular}{|c|c|c|c|}
\hline Input & System & Output & Problem Type \\
\hline $\begin{array}{c}\text { Known } \\
\text { (Detenninistic) }\end{array}$ & $\begin{array}{c}\text { Known } \\
\text { (Deterministic) }\end{array}$ & Unknown & Fonvard problem \\
\hline $\begin{array}{c}\text { Known } \\
\text { (Deteministic) }\end{array}$ & Inaccurate & $\begin{array}{c}\text { Known } \\
\text { (Deteministic) }\end{array}$ & $\begin{array}{l}\text { Model updating/ } \\
\text { Calibration }\end{array}$ \\
\hline $\begin{array}{c}\text { Known } \\
\text { (Deterministic) }\end{array}$ & Unknown & $\begin{array}{c}\text { Known } \\
\text { (Deterministic) }\end{array}$ & $\begin{array}{l}\text { System } \\
\text { identification }\end{array}$ \\
\hline $\begin{array}{c}\text { Known } \\
\text { (Deteministic) }\end{array}$ & Partially known & $\begin{array}{c}\text { Known } \\
\text { (Deteministic) }\end{array}$ & $\begin{array}{l}\text { Structural health } \\
\text { monitoring }\end{array}$ \\
\hline $\begin{array}{c}\text { Known } \\
\text { (Deterministic) }\end{array}$ & $\begin{array}{c}\text { Known } \\
\text { (Deteministic) }\end{array}$ & Prescribed & Control \\
\hline $\begin{array}{c}\text { Assumed } \\
\text { (Deterministic) }\end{array}$ & $\begin{array}{l}\text { Unknown } \\
\text { (Deterministic) }\end{array}$ & Prescribed & $\begin{array}{l}\text { Design } \\
\text { Optimization }\end{array}$ \\
\hline $\begin{array}{c}\text { Known } \\
\text { (Deterninistic) }\end{array}$ & $\begin{array}{l}\text { Known } \\
\text { (Stochastic) }\end{array}$ & Unknown & $\begin{array}{l}\text { Uncertainty } \\
\text { Quantification }\end{array}$ \\
\hline $\begin{array}{l}\text { Known } \\
\text { (Stochastic) }\end{array}$ & $\begin{array}{c}\text { Known } \\
\text { (Deteministic) }\end{array}$ & Unknown & $\begin{array}{l}\text { Uncertainty } \\
\text { Quantification }\end{array}$ \\
\hline $\begin{array}{l}\text { Known } \\
\text { (Stochastic) }\end{array}$ & $\begin{array}{l}\text { Known } \\
\text { (Stochastic) }\end{array}$ & Unknown & $\begin{array}{l}\text { Uncerlainty } \\
\text { Quantification }\end{array}$ \\
\hline $\begin{array}{c}\text { Known } \\
\text { (Stochastic) }\end{array}$ & Inaccurate & $\begin{array}{c}\text { Known } \\
\text { (Stochastic) }\end{array}$ & $\begin{array}{l}\text { Probabilistic } \\
\text { model updating }\end{array}$ \\
\hline Assumed & $\begin{array}{l}\text { Unknown } \\
\text { (Stochastic) }\end{array}$ & $\begin{array}{l}\text { Prescribed } \\
\text { (Stochastic) }\end{array}$ & $\begin{array}{l}\text { Design with } \\
\text { uncertainty }\end{array}$ \\
\hline
\end{tabular}

Figure 6: Domain of applicability of machine learning. Applications of machine learning are explained systematically in terms of three components, input, output and system. Machine learning can bring in the feasibility of analysis for all these problem types by effectively creating efficient predictive models of the actual systems.

development process. A model can have a very low error for the training data but still be inaccurate. For this purpose, a test dataset is used to evaluate the performance of the model on the basis of which final predictions are made. This is how the selection of the final model is done and the hypothesis function is also evaluated. Figure 5 shows the basic model representation for the initial implementation 
of machine learning. Detailed overview of sampling, ML model formation and accuracy checking, as discussed above, are provided later in this paper (refer to sections 3 - 5).

\subsection{Prediction, optimization and uncertainty quantification}

In polymer composites, machine learning has been majorly used for prediction of the material properties, process optimization, microstructural analysis, and quantification of uncertainties arising in the material and its properties due to the complex manufacturing processes. Figure 6 is a precise illustration of various situations in computational material science where ML can be applied. For example, consider a system of polymer composite beam subjected to load where the input parameters say the material properties of the beam are known, the load is known but the deflection caused due to the applied load is unknown. Here ML can be used to form an efficient predictive model mapping the deflection in terms of material properties and load, which can be used to obtain the deflection in a forward framework corresponding to any combination of values of the material properties and the load. The same ML model can also be utilized in an inverse framework for system identification, where the material properties can be obtained knowing the remaining two sets of information. In the following paragraphs, we provide a concise review of various such applications of machine learning in the context of polymer composites.

Pilania et al. [80] used density functional theory and machine learning approach to predict the atomization energy, lattice energy, dielectric constant and spring constant of various polymeric chains and found a good agreement of results in both the approaches. Daghigh et al. [81] used K - Nearest Neighbor, a machine learning algorithm to predict the heat deflection temperature of various biocomposites. Another research group [82] established a relationship between the property parameters of the carbon fiber and the macroscopic properties of the overall carbon fiber reinforced polymer composite using the decision tree model of machine learning. There is a variety of ML algorithms which are reported in the literature for their successful implementation in material science. This perplexing variety of machine learning algorithms makes optimization one of the most essential applications of ML. Optimization in simpler terms entails the process of exercising different models multiple times which is computationally very expensive and has the tendency of becoming intractable for complex simulations. Optimization algorithm is a process that involves iterative execution by comparing different models for possible solutions till a satisfactory result is found. Variables, constraints and objective functions are the three elementary keys of an optimization problem. Variables are the parameters that an algorithm can tune; constraints are the boundaries or limits within which these parameters must exist and the objective function is the goal towards which the algorithm 

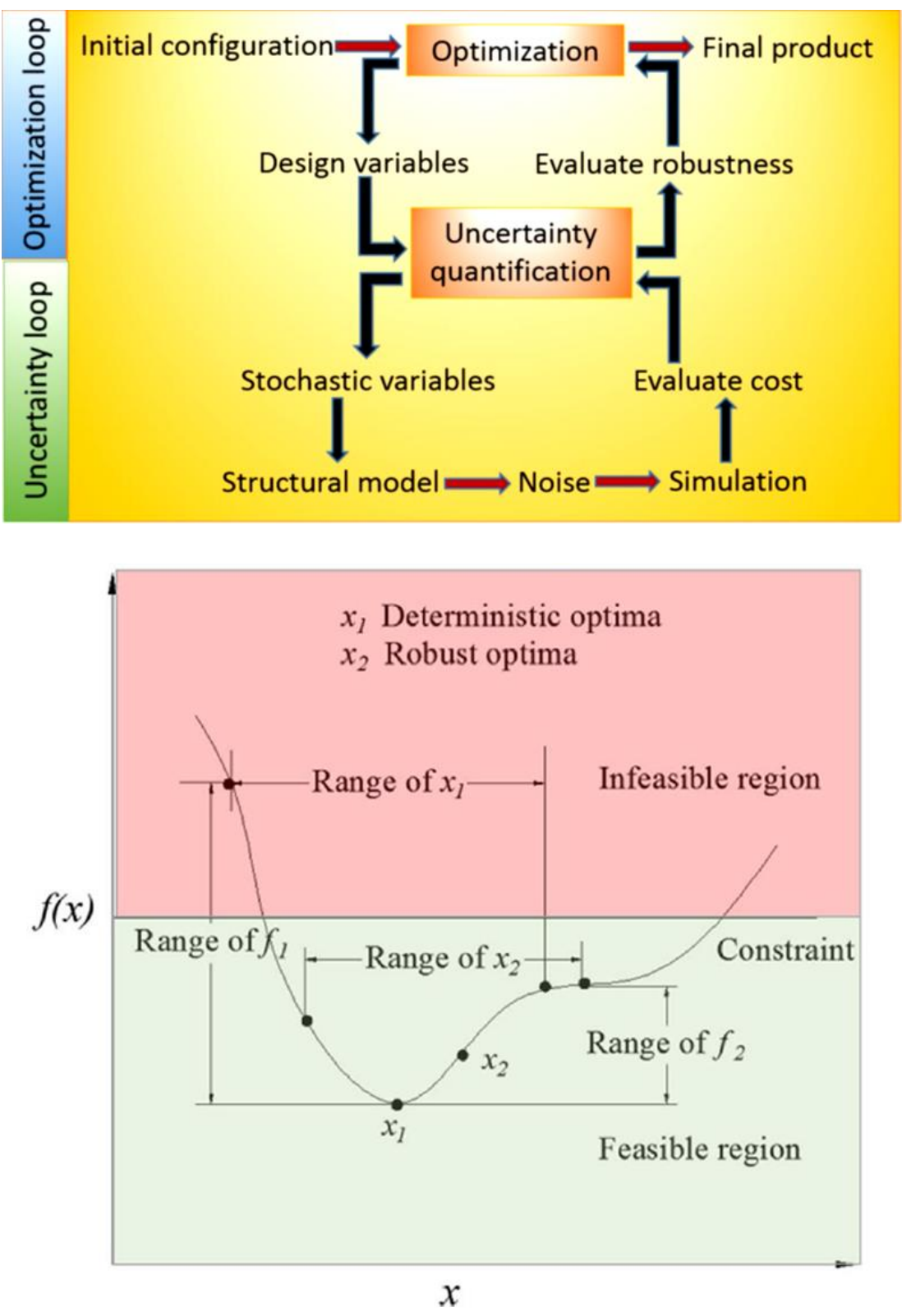

Figure 7: ML assisted robust design. Schematic illustration of robust design optimization that can be used for optimum system design while minimizing the propagation of uncertainty from the input to output space [86].

progresses. Stochastic and deterministic are the two different approaches widely used in optimization algorithms. Deterministic algorithms make use of particular rules to find the solution and the uncertainties in terms of variable space are ignored [83,84]. While the stochastic algorithms are more like probabilistic methods wherein the uncertainties are modeled with suitable probability distributions [85]. A new approach, called robust optimization, is also used to explicitly model and minimize the uncertainty involved in the problem. It makes use of a set-based deterministic description of the uncertainties [86]. Robust optimization creates a mathematical framework for the optimization which 


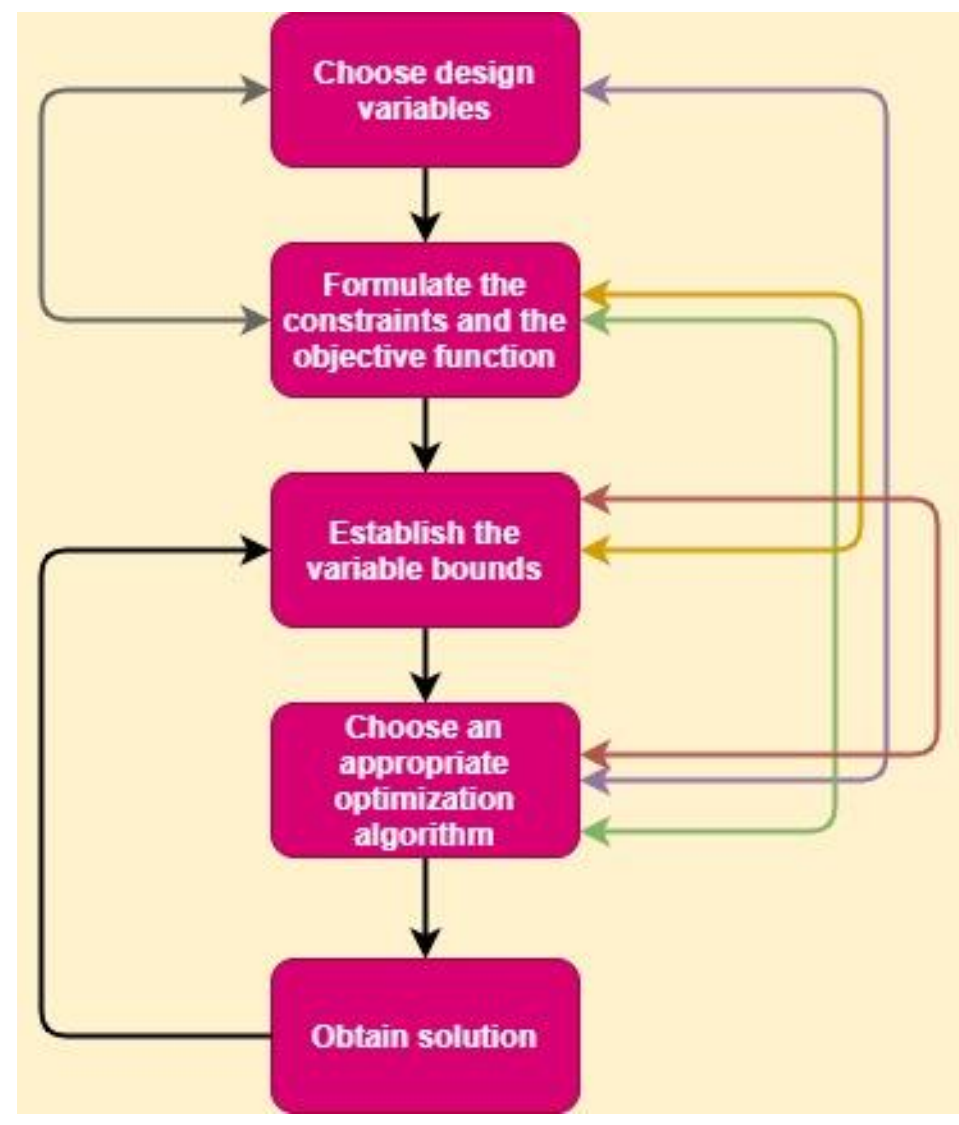

Figure 8: Flowchart for the optimal design. An optimization procedure can be used effectively to obtain optimal solutions concerning various types of engineering problems for choosing the right combination of input parameters.

minimizes the proliferation of uncertainties from the input space to the output responses [87], as depicted in Figure 7.

For the formulation of optimization problems, stochastic algorithms use random objective functions and constraints. Optimal design is achieved by comparing different possible hypothesis functions and then estimating each of their corresponding cost function (squared error function) by identifying the design variables and constraints. The entire optimal problem is then expressed in a mathematical form and is solved using an optimization algorithm. Figure 8 gives the flowchart of the basic procedure followed for the design of an optimal problem. Figure 9 shows the classification of optimization algorithms based on the design variables, objective function and constraints. Optimization methods can be significantly improved in terms of efficacy and efficiency by involving machine learning algorithms [88,89]. Salah et al. [90] predicted the absorption index of CNT reinforced polycarbonate composites using a ML approach of multilayer perceptron network approach. The optimization of the process parameters was performed and the best network was selected for the prediction of absorption index. Machine learning techniques have been extensively used to evaluate 


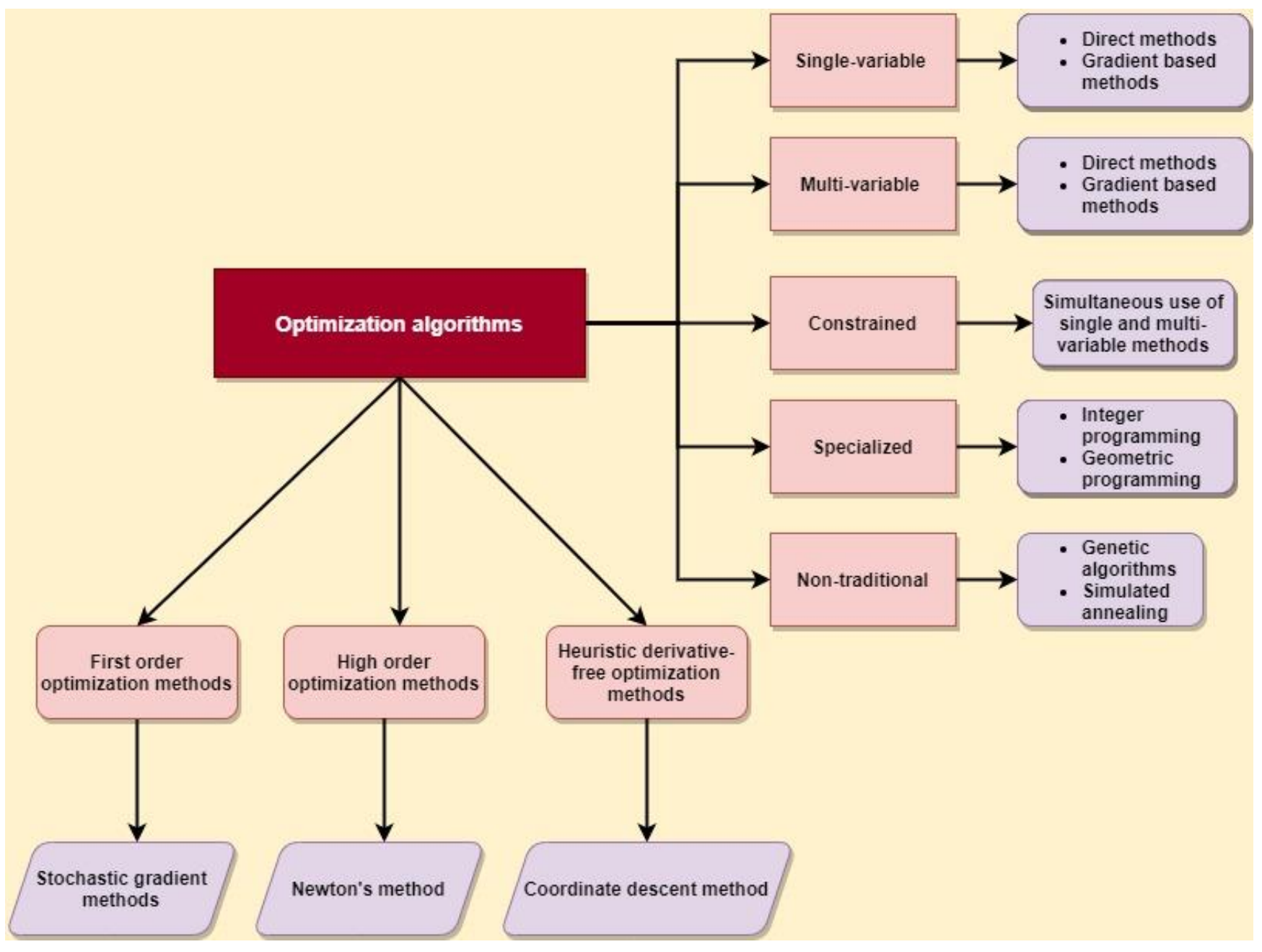

Figure 9: Classification of optimization algorithms. The optimization algorithms are classified based on the design variables, objective function and type of constraints.

and optimize the additive manufacturing processes [91]. Nik et al. [92] used a machine learning based optimization algorithm to find an optimal solution for the stiffness and the buckling load of polymer composite laminates. They successfully utilized the machine learning algorithm as an evolutionary model that had the capability of reducing the overall computational cost of the entire optimization process. Duan et al. [93] developed a multi-scale coarse-grained model using artificial neural network, a machine learning algorithm aided with particle swarm optimization, which was used to understand the inherent mechanisms of epoxy-based composites, facilitating the composite design with better performance. Various researchers have used machine learning algorithms to optimize the process parameters and characteristics like composite composition, delamination, surface roughness, thrust force, material properties and the overall structural design [94-99]. Li et al. [100] used a machine learning based iterative method to optimize the synthesis process of short fibers considering all the quantitative and qualitative objectives. This study demonstrated how the fiber synthesis process could 


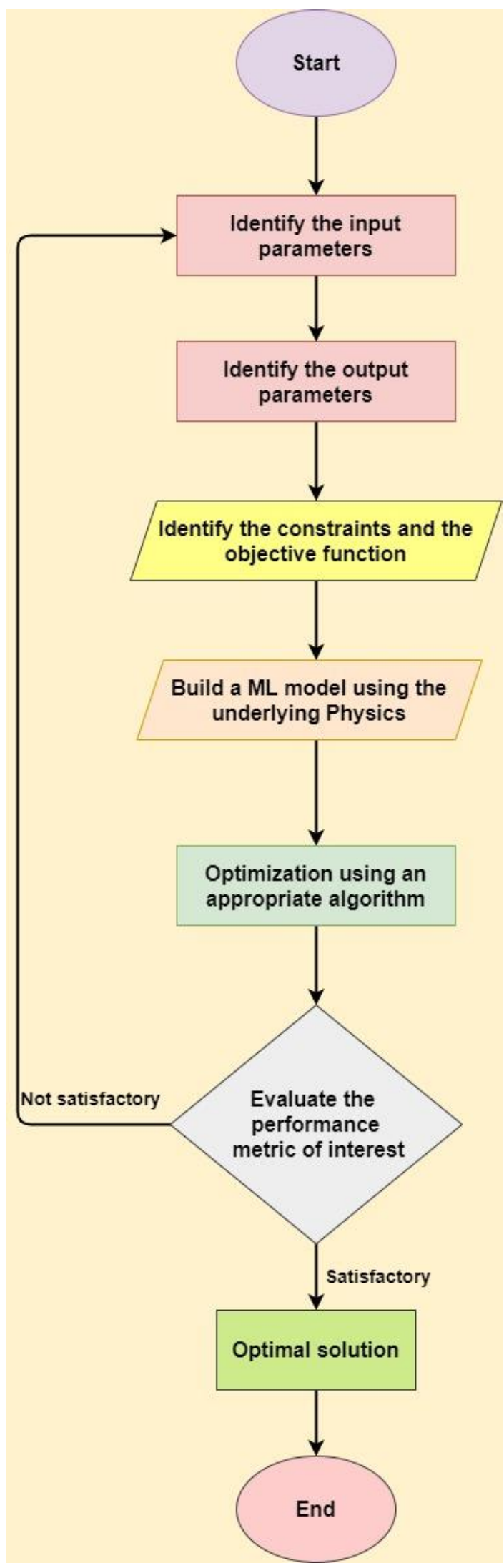

Figure 10: ML assisted optimization algorithm. A general flowchart is presented for using machine learning to solve optimization problems wherein more efficiency can be achieved and subsequently, the feasibility of exploring a design field can be substantially expanded for a better design outcome. 
be effectively focused on achieving the desired material properties. In material science, the quantification and representation of the microstructural design space are considered as one of the key research questions. Finding an appropriate descriptor set for the microstructural characterization of materials is of great importance. Xu et al. [101] proposed a ML based approach to identify the key microstructural descriptors and developed a four-step process to find the microstructural design variables considering the best descriptor among all. The quantity and morphology of the reinforcing fillers in the polymeric matrix affect the mechanical properties of the resulting composite to a large extent and hence makes microstructural optimization a significant research problem. A general flowchart of implementation of machine learning to solve optimization problems is given in Figure 10.

Computational analyses of polymer composites often encounter uncertainties because of the variations in the properties of the material, measurement uncertainty, limitations in the test set-up, operating environment and inaccurate geometrical features [102-106]. Uncertainty in parametric inputs, initial conditions and the boundary conditions, computational and numerical uncertainties arising from the unavoidable assumptions and approximations along with the inherent inaccuracy of the model result in major deviation from the deterministic values or the expected material behavior, altering the overall performance of composites. For ensuring that the simulation results are reliable and to understand the risks for making final product decisions, it is imperative to quantify these uncertainties. In the last few decades, researchers have made various attempts to quantify uncertainty [107-111], a brief review of which is given here. Bostanabad et al. [112] used the multi-response Gaussian process for uncertainty quantification in the simulation of woven fiber composites. They directly related the hyperparameters of the Gaussian process to the sources of physical uncertainty and reduced the overall computational cost. Doh et al. [113] used the approach of Bayesian inference to quantify the uncertainty of percolating electric conductance for the polymer nanocomposites reinforced with carbon nanotubes. They found that the correlation between the conductance of carbon nanotubes and the parameter of phase transition along with the critical exponent significantly affects the electrical conductance of the resulting composite in uncertainty quantification. ML models are trained on the available data and hence are prone to inherent uncertainties or errors associated with it. Jha et al. [114] considered the example of glass transition temperature to investigate the impact of uncertainties in the dataset on the predictions made by ML models. Using the Bayesian model they quantitatively represented the underlying uncertainties in the experimental values of the glass transition temperature. The methodology of Polynomial Chaos Expansion (PCE) is found to be extensively used to quantify the unavoidable uncertainties that exist in the matrix and the fiber which consequently affect the material properties and their global responses [115-117]. This expansion is also known as Wiener 


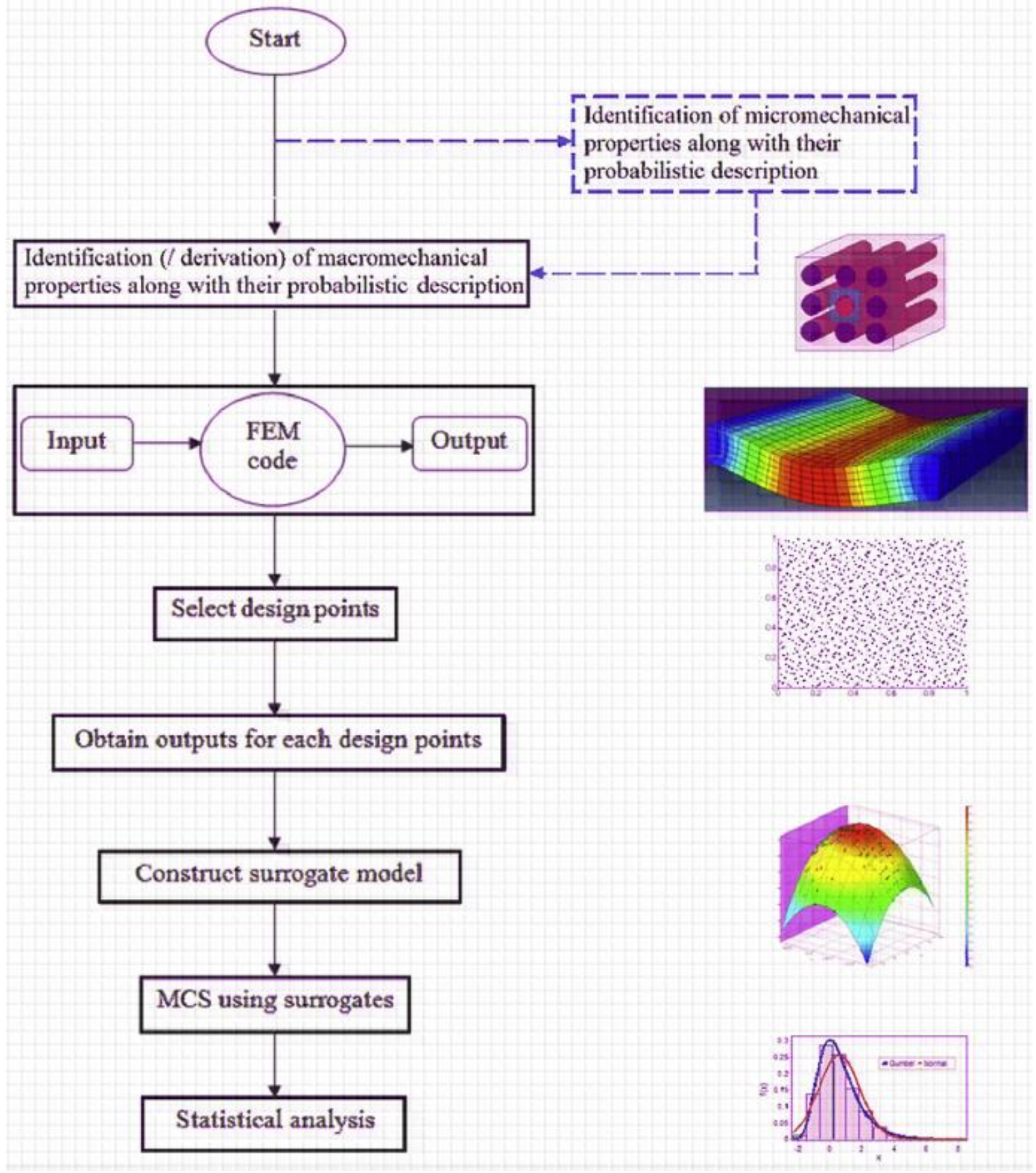

Figure 11: ML assisted multi-scale uncertainty quantification of polymer composites. Flowchart for machine learning assisted uncertainty quantification and stochastic macro and micro-mechanical analysis of laminated composites [135], wherein representative figures are given for finite element analysis, sobol's quasi-random sampling, machine learning based surrogate model formation and uncertainty quantification (corresponding to the respective steps).

Chaos expansion and is an effective technique for solving stochastic systems. In its most basic form, it is a way of representing random variables/processes in terms of orthogonal polynomials. The core concept about PCE is that it allows the random variables of any arbitrary distribution to be represented about a random variable of our choice. Mathematically it is of the following form, 


$$
X=\sum_{i=}^{\infty} X_{i} \varphi_{i}(Z)
$$

where $X$ is a random variable which is decomposed in terms of deterministic coefficients $X_{i}$ over an orthogonal polynomial basis $\varphi_{i}(Z)$. PCE has been used by many to handle the uncertainties in the input parameters. Thapa et al. [118] used the methodology of polynomial chaos expansion for quantifying the uncertainty in carbon-reinforced polymer composites. Keeping in view that the reliability and the overall performance of these composites depend on the properties of matrix and fiber along with the fiber orientation, loading conditions and the thickness of the resulting composite structure, uncertainty was considered in the stages of micro and macro scale modelling. The results obtained from the polynomial chaos decomposition were found to be computationally superior in fetching the vital information about polymer composites using a stochastic method. Dey et al. [119] used PCE as surrogate to analyze the propagation of uncertainties in the dynamic response of composite plates following a fuzzy based non-probabilistic framework. An efficient ML assisted algorithm for multiscale uncertainty quantification of polymer composites is presented in figure 11.

\subsection{Reliability and sensitivity analysis}

Polymer composites are being increasingly utilized by aerospace, automotive, marine and many other technologically advanced industries, where performance reliability is an important criteria for the product design. For sustainable development, robust design and safe operation of complex systems, it is essential to evaluate the reliability of these systems including identification of anomalies [120]. Production and development of large scale polymer composites involve utilization of dicey materials and manufacturing processes. This makes reliability analysis an integral part of material design. Considering numerous structural and non-structural applications of polymer composites and the different types of loading that these composite products are subjected to during their service period, it is imperative to perform a reliability analysis for their safe industry applications. Lately, to evaluate the reliability of complex systems, ML based methodology has been used by researchers for addressing the problems in a computationally efficient framework [121,122]. Behnia et al. [123] developed a robust reliability analysis and damage statement index by making use of parametric acoustic emission analysis. They integrated the bathtub curve and Weibull function to develop a hazard rate and used support vector regression for failure prediction. Alsina et al. [124] conducted a comparative study for the reliability analysis of engineered products. They considered different ML models like artificial neural networks, random forest and support vector machine, wherein it was found that random forest outperformed all other approaches. They also concluded that ML algorithms have better prediction 
capability compared to the traditional approaches. Yan et al. [125] used artificial neural network and genetic algorithm to assess the anchorage reliability of bars made up of Glass Fiber Reinforced Polymer (GFRP) composite. Using this ML methodology they successfully determined the reliability index and the failure probability of GFRP bars. In an effort to reduce the computational cost of reliability analysis, Tan et al. [126] used radial basis function in conjunction with support vector machine to estimate structural reliability. Another research group [127] used artificial neural network for the reliability analysis of strength models so as to predict the confinement strength of concrete with Carbon Fiber Reinforced Polymer (CFRP), leading to the proposition of a new empirical equation based on available experimental data. A close agreement was found between the predicted and the experimental results. Multiple successful attempts to carry out reliability analysis using the approach of machine learning can be traced in literature [128-131].

For developing an efficient system, it is very important to have an appropriate insight into the variables affecting it. As discussed in the preceding section, a number of different parameters affect the ultimate behavior of polymer composites; considering the uncertainties, manufacturing conditions and assumptions in these parameters, sensitivity analysis is of prime importance. Sensitivity analysis helps to determine how the final behavior of polymer composites (output variable) will be affected if there are any changes in the parameters affecting it (input variables). Such analysis plays a crucial role in ensuring the robustness of polymer composites. It can be noted that multiple studies can be found in literature where the performance of sensitivity analysis has been improved by integrating ML with the algorithm [111,132]. Karsh et al. [133] proposed a ML based sensitivity analysis methodology to assess the properties of functionally graded materials considering the variations along the depth of functionally graded plates. They performed moment independent sensitivity analysis with polynomial chaos expansion based on least angle regression to account for the effect of varying the material properties, boundary and loading conditions on the impact response and natural frequencies. Using this sensitivity analysis method they found out the most and the least sensitive parameters while achieving a significant level of computational efficiency. Dey et al. [134] and Naskar et. al. [135] performed variance-based sensitivity analyses at macro and micro levels in conjunction with machine learning for polymer composite laminates, wherein it was reported that fiber orientation angle is highly sensitive to the natural frequencies, followed by the material properties.

\section{Sampling techniques for training machine learning models}

To build an efficient machine learning model and analyze data, it is absolutely necessary to select the right subset of data based on which the 'learning' part will take place for a machine learning 
model. The selected subset should be optimally identified and perfectly representative of the entire analysis domain. It should not miss any of the important features that may govern the resulting behavior of the material. Such sampling techniques facilitate one with reliable information at a much lesser computational cost. For selecting the data subset from the distribution of interest, various sampling techniques are used to ensure that the selected subset is free from selection and measurement bias. The successful development of any ML model depends largely on the experimental design used for obtaining the learning data [136]. To estimate a function or a system that is computationally expensive, it is required to generate optimal sample points that can represent the entire analysis domain when integrated with an appropriate machine learning algorithm [137,138].

The sample points can be generated using various available sampling algorithms like Central Composite Design (CCD) [139,140], Latin hypercube design [141], uniform design [142], D-optimal design [143], Hammersley sequences [144] and orthogonal array sampling [145,146]. Alam et al. [147] compared a few of these sampling techniques in order to study the impact that experimental design has on the development of a neural network model. They found the best performance corresponding to the model developed using the Latin hypercube design technique. Central composite design lacks in its efficacy when it comes to a design problem having high-dimensional data [148]. In an attempt to achieve complete uniform curing along with reducing the residual stresses in the case of a fiberreinforced composite laminate, Shah et al. [149] used a genetic algorithm and Latin hypercube sampling technique to optimize the parameters of the curing process. The positive results of this study encouraged manufacturing engineers to make use of the proposed approach. Johnston et al. [150] used Latin hypercube sampling technique and the conventional Monte Carlo simulation to model the uncertainties in the volume fraction of the fiber and viscoplastic material constant. A comparison was made between both the sampling techniques and the former was found to have a better correlation with the experimental data. Another research group [151] investigated the tribological properties of glass fiber reinforced polymer composites strengthened with filler materials like silicon carbide and graphite particles. They used the sampling technique of orthogonal arrays to obtain the right experimental design and subsequently applied analysis of variance to study the effect of various process parameters on the wear behavior of polymer composites. Ghelich et al. [152] used central composite design methodology to fit around 50 electrospinning experimental data and investigated the effect of process parameters on the quality and uniformity of resulting nanocomposites. The methodology of orthogonal array was used to optimize the reactivity of alkali-treated circulating fluidized bed combustion fly ash [153]. A comparative assessment of different sampling techniques always provides a clear perspective regarding the performance of a machine learning model. Mukhopadhyay et al. established that D- 
optimal design and central composite design performs better compared to other design of experiment (DoE) methods for composite laminates [140,154]. Later the same group extended their study concerning the prediction and identification problems relate to web-core composite panels to report that the quasi-random sobol sequence outperforms other sampling techniques like Halton, Latine hypercube and random uniform [155]. Using an appropriate and optimal sampling technique to build machine learning models often results in efficient predictions and lesser computational effort [154,156-158].

\section{Widely adopted machine learning algorithms in polymer composites}

In the pursuit of making advancement in polymer composites by means of discovering novel materials, tailoring application-specific properties of these materials and evaluating the various affecting parameters so as to come up with an optimized design, researchers are continuously investigating the exploitation of the growing capabilities of machine learning algorithms. Such research activities have resulted in many successful attempts which are summarized in the following subsections considering the most widely adopted machine learning algorithms.

\subsection{Neural networks}

Neural network is an iterative approach that is the most preferred algorithm by many material science researchers to investigate various data-intensive aspects, which forms the basis of many key advancements in the field of artificial intelligence over the last few decades. It is a mathematical tool which is inspired by the biological nervous system and is used to solve a wide range of engineering and scientific problems by recognizing the underlying relationships in the available data [159]. In the human brain, there is a gazillion of neurons connected together within a network which helps in processing the flow of information to generate meaningful outputs. Similarly in neural networks, there are number of neurons that act as processors operating in parallel and arranged in different layers. The first layer receives all information (preprocessed data) to be considered and is known as the input layer. Then there is an intermediate layer which is commonly called the hidden layer. It contains many discreet nodes and is responsible for all the computations [160]. The final layer, known as the output layer, provides the final results of the prediction. Figure 12 shows the basic architecture of Artificial Neural Network (ANN). There are estimation algorithms within the network that assign synaptic weights to the input parameters and then calculate the output. Robustness and efficiency of this method lie in its ability to handle a large amount of data with huge covariate spaces by making use of nonlinear mapping functions [161]. Multilayer Perceptron (MLP) and Radial Basis Function (RBF) are predictor functions which are frequently used in artificial neural networks [162]. These predictor functions help

to minimize the error in the prediction of outputs. Feedforward architecture with backward propagation 
is usually followed for the output computation and error minimization. In feedforward architecture, no loops are formed in the entire network. Information in any of the units of the successive layers does not receive any feedback. But in case of back propagation, synaptic weights are adjusted by back propagating the error [163]. Weights are updated after each record is run through the network. One iteration is completed when all the records complete running through the network and it is technically known as epoch. The process is repeated after completing one epoch. There are mathematical equations (activation functions) for linking the weighted sums of each layer with the succeeding layer and deliver the output. The architecture of ANN model can be mathematically defined as

$$
\begin{gathered}
Y=F(x) \\
Y=A\left(W \times x+B_{i}\right)+B_{O}
\end{gathered}
$$

where $Y$ is the output vector, $x$ is the input vector, $A$ is the activation function (sigmoid, Tanh, softmax, softplus etc.), $W$ is the matrix that contains the synaptic weights, $B_{i}$ is the column vector of biases from the input layer to the hidden layer and $B_{O}$ is the column vector of biases from the hidden layer to the output layer.

ANN is one of the most used ML algorithms for the purpose of data classification, prediction, clustering, pattern and image recognition [164,165]. The successful applications of ANN in diverse fields include prediction of economic stability, remote sensing, weather forecasting, estimation of crop production and in the areas of medicine, environment, mining, materials and nanotechnology [166168]. Owing to the efficacy of this function approximation method, it has been used to predict the mechanical properties of various composite materials with little experimental effort [169-172]. Matos et al. [173] used the framework of artificial neural network to explore the possibility of using polymer composites reinforced with Carbon Nano Tubes (CNT) in the production of different types of damage detecting sensors. They produced extensive data by making use of Finite Element Method (FEM) and trained the neural network using it. As a result, they predicted the conductivity of the composite material as a function of multiaxial strain. Another important observation of this study was the major saving in the computational time (> 6 orders of magnitude). Each simulation performed using finite element method required 3.5 hours to run, while ANN made it possible to get the same output in less than 0.2 seconds. Neural networks have been considered as a novel technique to predict the tribological characteristics of polymer composites [174,175]. Many researchers have successfully used neural networks to predict and optimize the wear rate, load carrying capacity, damage-deformation and sound insulation properties of various polymer composites [176-181]. Jiang et al. [182] applied ANN for predicting the wear behavior of polymer composites as a function of the composition and test 


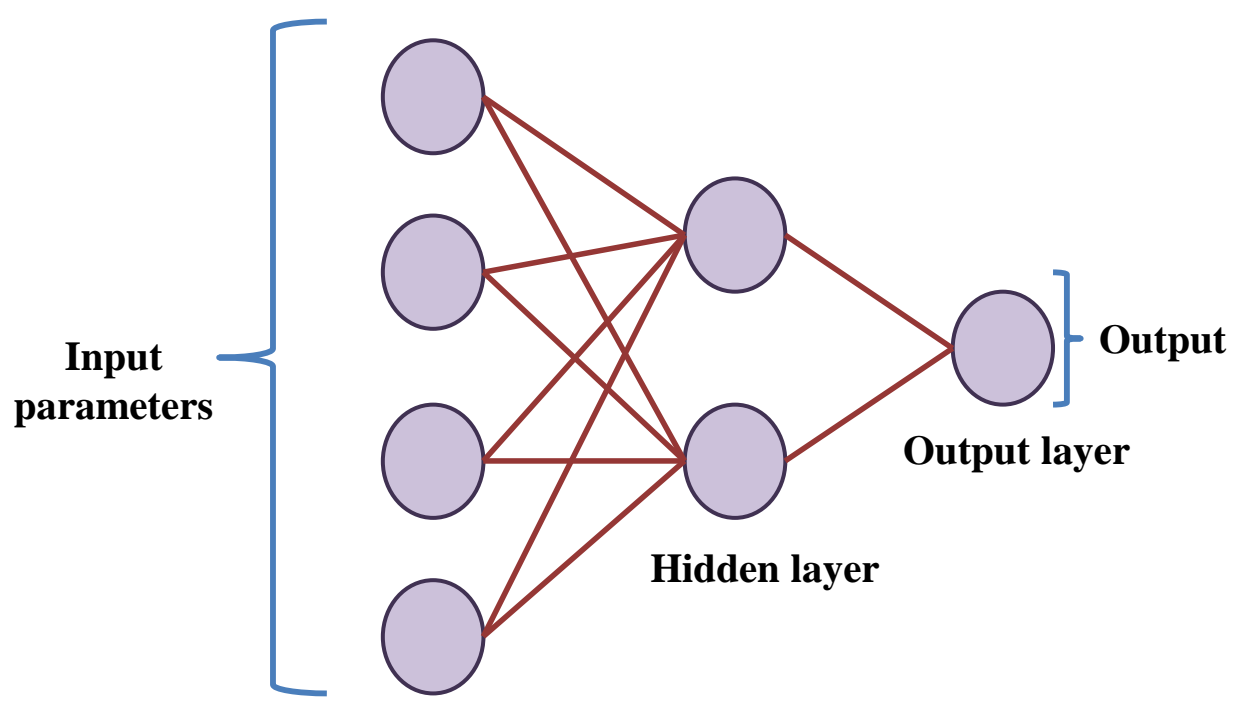

Input layer

(a)

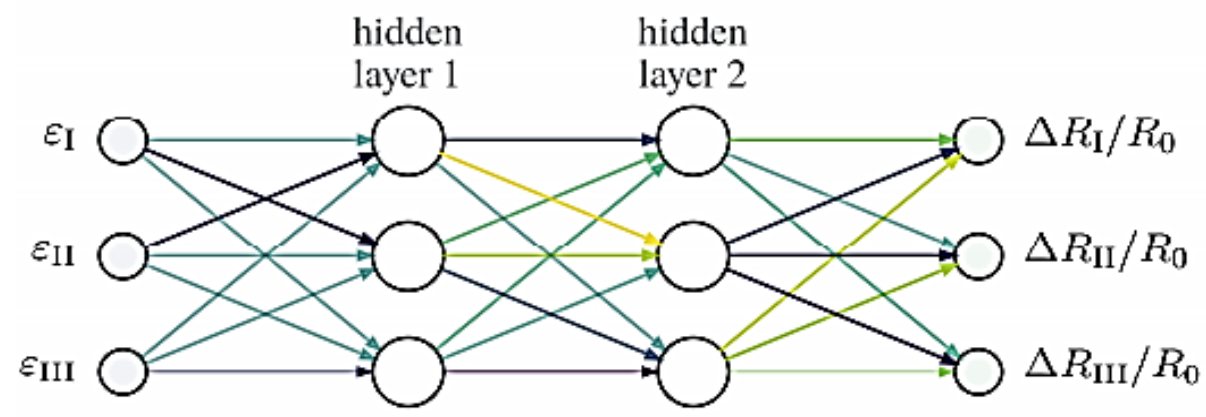

(b)

Figure 12: Basic architecture of artificial neural network (ANN). (a) A typical representation of the ANN architecture having input, hidden and output layers to receive the preprocessed data, compute the correlations and give the predicted output, respectively. (b) Artificial neural networks for predicting the electro-mechanical response of CNT reinforced polymer composites [166].

conditions. They tried training the neural network using different types of algorithms and found that Powell-Beale Conjugate Gradient Algorithm (CGB) provided the best results in terms of accuracy and computational time. Along with the wear behavior, predictions for compressive and flexural strength were also carried out. It was a successful attempt of predicting the behavior of glass reinforced composites at different exposure conditions with limited experimentation.

ANN based machine learning techniques have been efficaciously used to model the process parameters by establishing a correlation between the various factors affecting that process. Guo et al. [183] correlated the parameters of induction welding process with the temperature varying in the 
overlapping zone of laminates made up of carbon fiber reinforced polyetherketoneketone composites using neural networks. To train the network, data was obtained from two sets of experiments. One set of the experiments was performed using a vacuum bag and the other was done using a KVE tool (a thermoplastic assembly platform). Temperature sensors and fiber optic were installed in the overlapping region to record the variation in the temperature field when induction heating was done for a particular time period. This experimental data was then processed before feeding to the network so as to have temperature-time consistency. Two neural networks were used with the same architecture separately for the two datasets obtained from the above-mentioned sets of experiments. Accuracy of the used network was tested based on the difference between the experimental and the predicted value. The proposed neural network could model the induction welding process and reliable predictions of temperature variation were made. Vacuum-assisted resin transfer molding is one of the widely used processes for manufacturing polymer composites and the properties of such manufactured composites vary from the ones undergone any other manufacturing process. In this context, Seyhan et al. [184] used a feed-forward ANN network to predict the compressive strength of glass-reinforced polyester composites and explained the effect of using the above-mentioned manufacturing process. In this study, 30 datasets were used for the training step and 15 sets were used for the testing purpose. A total of 100000 iterations were set using a code in $\mathrm{C}++$. Predicted results were compared with the results obtained corresponding to a multi-linear regression model and ANN showed better results. Composite structures are usually connected by means of different fasteners and adhesives. These connections are often prone to damage and defects when subjected to load. Neural networks have proven their potential in accurately detecting the damage in the connections of composite materials. Farhangdoust et al. [185] successfully used ANN with heterodyne effect methodology to locate the defects in the connection zone of the polymer composite where the linear characteristics change into non-linear ones.

Adaptive neuro fuzzy network is another type of artificial neural network which is based on fuzzy inference system. It is known as a universal estimator as it has the benefits of neural networks as well as fuzzy logics and integrates the principles of both in a single architecture. Researchers have implemented ANN and neuro fuzzy inference system to predict the impact strength, yield strength and other mechanical properties of glass reinforced polymer composites, and concluded that both ANN and fuzzy inference system can be successfully implemented to any type of polymer composites to predict the mechanical behavior [186]. Neural networks have been successfully applied for exploring the fatigue response of polymer composites under different conditions. Mini et al. [187] made use of three different paradigms of ANN (feedforward, recurrent and radial basis function in conjunction with FEM to train the network) to predict the fatigue strength of polymer composites. Later the predicted results 
were compared with the available experimental values and the network of radial basis function was found to obtain the most accurate results. Hassan et al. [188] investigated the fatigue behavior of various shafts made up of polyester based composite material under the action of bending. They made use of ANN to predict the fatigue life of these shafts as a function of fiber properties and the ultimate bending stress. The number of nodes in the hidden layer was varied and based on the least root mean square error, the optimized ANN structure was selected. Vassilopoulos et al. [189] made use of ANN to predict the number of cycles before failure for Glass Fiber Reinforced Polymer (GFRP) composites. They came up with S-N curves and Constant Life Diagrams (CLD) by making use of only 50\% experimental data. These diagrams were further utilized for designing purposes. Ramasamy et al. [190] used a feed forward ANN to predict the behavior of GFRP composites when subjected to drop impact test. Data fed to the ANN model was experimentally obtained by conducting a drop impact test and acoustic emission technique, wherein Purlin adaptive learning function along with the gradient descent algorithm was used.

Polynomial Neural Network (PNN) is a class of neural frameworks that has recently gained popularity in composites for the prediction of material behavior. Predictive modelling based on polynomial neural networks is found to be more accurate compared to other fuzzy models [191]. PNNs are most preferred when the output function is a higher order polynomial of the input function. Assaf et al. [192] modeled the fatigue behavior of glass fiber reinforced polymer composites using the feedforward recurrent neural networks and polynomial classifiers to compare the results of both the networks. Orientation angle of the fibers, R-ratio and ultimate stress were used as the input parameters in both the cases, where polynomial classifiers were found to be more accurate in predicting the fatigue life of these composites. It was noted that the predictions from both the approaches were comparable with the other existing fatigue life prediction techniques. Kumar et al. [193] used polynomial neural networks to model the buckling behavior of sandwich plates. The results obtained using the PNN model were found to be in line with the results of Monte Carlo simulation.

Another important class of neural networks is Convolutional Neural Network (CNN) which falls under the category of deep learning. Researchers these days are effectively making use of CNN for discovering new materials with optimal performance. It is basically an algorithm that has been successfully used for analyzing images and coming out with meaningful results [194-196]. Abueidda et al. [197] developed a CNN model to quantitatively predict the mechanical behavior of composites by making use of different grid sizes of the composite's microstructure obtained from Scanning Electron Microscope (SEM). Using the chemical structure of different polymers, convolutional neural network made it possible to predict the glass transition temperature along with other polymeric properties 
Table 1. List of studies concerning polymer composites conducted using neural networks

\begin{tabular}{|c|c|c|c|c|c|}
\hline $\begin{array}{l}\text { Refer } \\
\text { ence } \\
\text { No. }\end{array}$ & Material & $\begin{array}{r}\text { Source of } \\
\text { database }\end{array}$ & ML model & Input & Output \\
\hline [173] & $\begin{array}{l}\text { CNT-polymer } \\
\text { composites }\end{array}$ & $\sum_{\text {至 }}$ & ANN & Principal strains & $\begin{array}{c}\text { Material } \\
\text { conductivity }\end{array}$ \\
\hline [182] & $\begin{array}{l}\text { Short fiber reinforced- } \\
\text { polyamide composites }\end{array}$ & 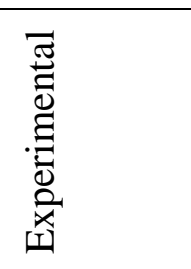 & ANN & $\begin{array}{c}\text { Material } \\
\text { composition, } \\
\text { temperature, sliding } \\
\text { speed, normal load }\end{array}$ & $\begin{array}{c}\text { Mechanical } \\
\text { properties and } \\
\text { wear } \\
\text { characteristics }\end{array}$ \\
\hline [184] & $\begin{array}{l}\text { Glass fiber reinforced } \\
\text { polyester composites }\end{array}$ & 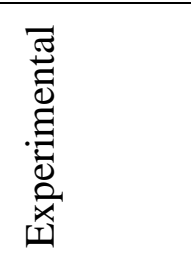 & ANN & $\begin{array}{l}\text { Amount of binder } \\
\text { and fiber used + } \\
\text { fiber thickness }\end{array}$ & $\begin{array}{l}\text { Compressive } \\
\text { strength }\end{array}$ \\
\hline [186] & $\begin{array}{l}\text { Glass fiber reinforced } \\
\text { polymer composites }\end{array}$ & 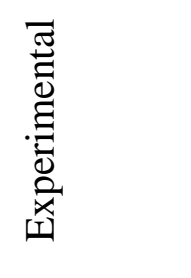 & $\begin{array}{c}\text { ANN + } \\
\text { Adaptive } \\
\text { Neuro Fuzzy } \\
\text { Inference } \\
\text { System }\end{array}$ & $\begin{array}{l}\text { Fiber content, } \\
\text { mixing temperature, } \\
\text { feed rate }\end{array}$ & $\begin{array}{c}\text { Mechanical } \\
\text { properties }\end{array}$ \\
\hline [187] & $\begin{array}{c}\text { Glass fiber reinforced } \\
\text { epoxy composites }\end{array}$ & 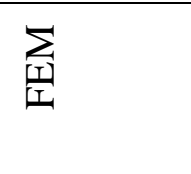 & ANN & $\begin{array}{c}\text { Fiber orientation, } \\
\text { ultimate stress, stress } \\
\text { ratio }\end{array}$ & $\begin{array}{l}\text { Number of } \\
\text { cycles analogue } \\
\text { to failure }\end{array}$ \\
\hline [188] & $\begin{array}{c}\text { Glass reinforced } \\
\text { polyester composites }\end{array}$ & 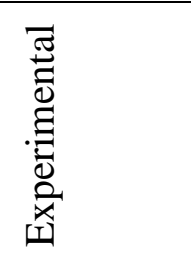 & ANN & $\begin{array}{l}\text { Type of fiber, } \\
\text { volume fraction, } \\
\text { bending stress }\end{array}$ & Fatigue life \\
\hline [190] & $\begin{array}{c}\text { Glass reinforced } \\
\text { polymer laminates }\end{array}$ & 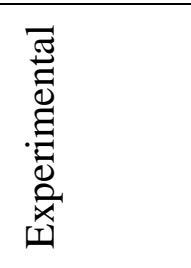 & ANN & $\begin{array}{c}\text { Parameters of } \\
\text { acoustic emission }\end{array}$ & $\begin{array}{c}\text { Tolerance to } \\
\text { impact damage }\end{array}$ \\
\hline [197] & Polymer composites & 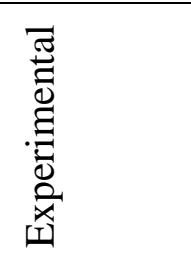 & $\mathrm{CNN}$ & $\begin{array}{l}\text { Composite's } \\
\text { microstructure }\end{array}$ & $\begin{array}{c}\text { Compressive } \\
\text { strength and } \\
\text { stiffness }\end{array}$ \\
\hline
\end{tabular}




\begin{tabular}{|c|c|c|c|c|c|}
\hline [199] & $\begin{array}{c}\text { Carbon Fiber } \\
\text { Reinforced Plastics } \\
\text { (CFRP) }\end{array}$ & 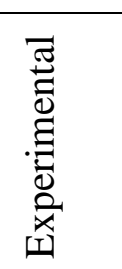 & ANN & $\begin{array}{l}\text { Feed rate, spindle } \\
\text { speed and point } \\
\text { angle }\end{array}$ & $\begin{array}{l}\text { Delamination } \\
\text { factor }\end{array}$ \\
\hline [200] & $\begin{array}{l}\text { Short glass }+ \text { carbon } \\
\text { reinforced polyamide } \\
\text { composites }\end{array}$ & 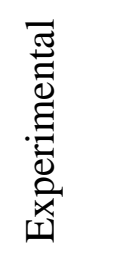 & ANN & $\begin{array}{c}\text { Material } \\
\text { composition, } \\
\text { mechanical } \\
\text { properties and tribo } \\
\text { test conditions }\end{array}$ & $\begin{array}{l}\text { Wear rate and } \\
\text { coefficient of } \\
\text { friction }\end{array}$ \\
\hline [201] & $\begin{array}{c}\text { Carbon fiber reinforced } \\
\text { polyetheretherketone } \\
\text { composites }\end{array}$ & 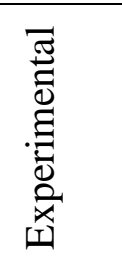 & ANN & $\begin{array}{l}\text { Mechanical factor } \\
\text { (pv), Contact } \\
\text { temperature }\end{array}$ & $\begin{array}{l}\text { Wear weight } \\
\text { loss and } \\
\text { coefficient of } \\
\text { friction }\end{array}$ \\
\hline [202] & $\begin{array}{l}\text { Glass fiber reinforced } \\
\text { acrylonitrile butadiene } \\
\text { styrene filament }\end{array}$ & 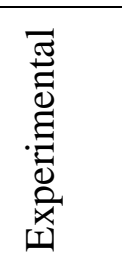 & $\begin{array}{l}\text { Recurrent } \\
\text { Neural } \\
\text { Network }\end{array}$ & $\begin{array}{l}\text { Micro CT-scan and } \\
\text { SEM images }\end{array}$ & $\begin{array}{c}\text { Fiber } \\
\text { orientation }\end{array}$ \\
\hline [203] & $\begin{array}{c}\text { Particles, fibers and } \\
\text { nanowires }\end{array}$ & 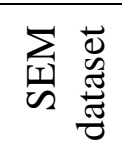 & $\mathrm{CNN}$ & SEM images & $\begin{array}{c}\text { Image } \\
\text { classification }\end{array}$ \\
\hline [204] & $\begin{array}{l}\text { Glass fiber reinforced } \\
\text { plastic composites }\end{array}$ & 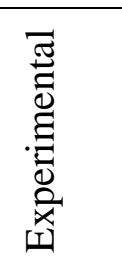 & ANN & $\begin{array}{l}\text { End milling cutting } \\
\text { parameters }\end{array}$ & Damage factor \\
\hline [205] & $\begin{array}{l}\text { Carbon fiber reinforced } \\
\text { polymer composites }\end{array}$ & 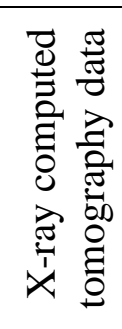 & CNN & 3D image data & $\begin{array}{l}\text { Identification of } \\
\text { delamination in } \\
\text { the composites }\end{array}$ \\
\hline [206] & $\begin{array}{c}\text { Carbon fiber composite } \\
\text { panel }\end{array}$ & 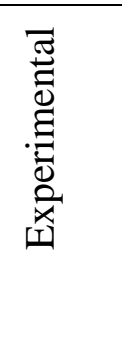 & ANN & $\begin{array}{l}\text { Acoustic emission } \\
\text { parameters like rise } \\
\text { time, absolute } \\
\text { energy, counts, } \\
\text { amplitude and } \\
\text { frequency }\end{array}$ & $\begin{array}{c}\text { Damage } \\
\text { characterization } \\
\text { in buckling }\end{array}$ \\
\hline
\end{tabular}




\begin{tabular}{|c|c|c|c|c|c|}
\hline [207] & $\begin{array}{l}\text { Silica filled epoxy } \\
\text { composites }\end{array}$ & 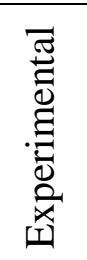 & ANN & $\begin{array}{l}\text { Aspect ratio, volume } \\
\text { fraction, time and } \\
\text { elastic modulus }\end{array}$ & $\begin{array}{c}\text { Stress intensity } \\
\text { factor }\end{array}$ \\
\hline [208] & $\begin{array}{l}\text { Polymer composites } \\
\text { reinforced with glass }\end{array}$ & 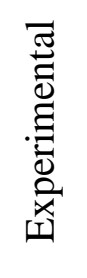 & ANN & $\begin{array}{l}\text { Elastic modulus, } \\
\text { shear wave speed, } \\
\text { longitudinal wave } \\
\text { speed, time, volume } \\
\text { fraction, density }\end{array}$ & $\begin{array}{l}\text { Fracture } \\
\text { behavior }\end{array}$ \\
\hline [209] & $\begin{array}{c}\text { Glass reinforced } \\
\text { polymer composites }\end{array}$ & 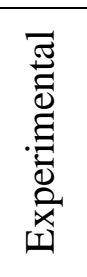 & ANN & $\begin{array}{l}\text { Mechanical } \\
\text { properties, fractal } \\
\text { dimension }\end{array}$ & Fatigue damage \\
\hline [210] & $\begin{array}{l}\text { Glass fiber reinforced } \\
\text { epoxy composites filled } \\
\text { with red brick dust }\end{array}$ & 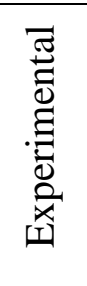 & ANN & $\begin{array}{l}\text { Impact velocity, } \\
\text { impingement angle, } \\
\text { size of erodent, brick } \\
\text { dust content, } \\
\text { temperature }\end{array}$ & $\begin{array}{l}\text { Erosion wear } \\
\text { rate }\end{array}$ \\
\hline [211] & $\begin{array}{l}\text { Mud filled polyester } \\
\text { matrix composites }\end{array}$ & 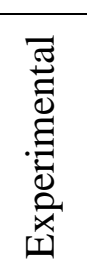 & ANN & $\begin{array}{l}\text { Sliding velocity, } \\
\text { normal load, mud } \\
\text { content }\end{array}$ & $\begin{array}{l}\text { Sliding wear } \\
\text { rate }\end{array}$ \\
\hline [212] & $\begin{array}{c}\text { Carbon fiber }+\mathrm{TiO}_{2} \\
\text { reinforced } \\
\text { polytetrafluroethylene } \\
\text { composites }\end{array}$ & 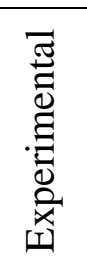 & ANN & $\begin{array}{l}\text { Tribo test conditions } \\
\text { and material } \\
\text { composition }\end{array}$ & $\begin{array}{l}\text { Wear volume } \\
\text { loss and } \\
\text { coefficient of } \\
\text { friction }\end{array}$ \\
\hline [213] & $\begin{array}{l}\text { PA-46 composites } \\
\text { reinforced with } \\
\text { carbon/glass fillers }\end{array}$ & 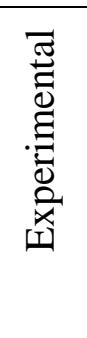 & ANN & $\begin{array}{l}\text { Average load, } \\
\text { velocity, } \\
\text { compressive } \\
\text { strength, modulus, } \\
\text { impact strength, } \\
\text { testing conditions }\end{array}$ & Wear volume \\
\hline
\end{tabular}




\begin{tabular}{|c|c|c|c|c|c|}
\hline [214] & Polymeric bearings & 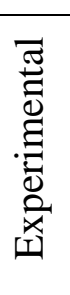 & ANN & $\begin{array}{l}\text { Load, viscosity, } \\
\text { sliding distance, } \\
\text { sliding velocity, } \\
\text { coefficient of } \\
\text { friction, temperature }\end{array}$ & Wear volume \\
\hline [215] & $\begin{array}{l}\text { Polymer composites } \\
\text { filled with nano-silica }\end{array}$ & 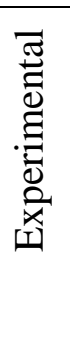 & ANN +ANFIS & $\begin{array}{l}\text { Filler volume } \\
\text { fraction, filler size, } \\
\text { elastic modulus and } \\
\text { the yield strength of } \\
\text { the matrix, fracture } \\
\text { energy of the matrix }\end{array}$ & $\begin{array}{l}\text { Fracture energy } \\
\text { of the polymer } \\
\text { nanocomposite }\end{array}$ \\
\hline [216] & $\begin{array}{l}\text { Continuous fiber } \\
\text { reinforced composites }\end{array}$ & 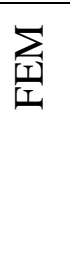 & ANN & $\begin{array}{l}\text { Sample thickness, } \\
\text { coefficient of } \\
\text { friction, residual } \\
\text { stress, interface } \\
\text { toughness }\end{array}$ & $\begin{array}{c}\text { Load- } \\
\text { displacement } \\
\text { behavior }\end{array}$ \\
\hline [217] & $\begin{array}{l}\text { Polyphenylene sulfide } \\
\text { composites }\end{array}$ & 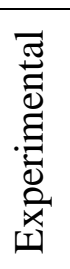 & ANN & $\begin{array}{c}\text { Mechanical and } \\
\text { thermos-mechanical } \\
\text { parameters }\end{array}$ & $\begin{array}{l}\text { Sliding wear } \\
\text { and friction } \\
\text { properties }\end{array}$ \\
\hline [218] & GFRP laminates & 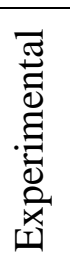 & ANN & $\begin{array}{c}\text { Acoustic emission } \\
\text { parameters }\end{array}$ & $\begin{array}{l}\text { Residual } \\
\text { strength }\end{array}$ \\
\hline [219] & $\begin{array}{l}\text { Carbon/glass/kevlar } \\
\text { fiber reinforced } \\
\text { polymer composites }\end{array}$ & 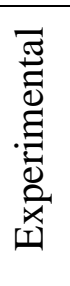 & ANN & $\begin{array}{l}\text { Incident velocity, } \\
\text { laminate } \\
\text { configuration, } \\
\text { Material } \\
\text { composition }\end{array}$ & $\begin{array}{l}\text { Projectile } \\
\text { residual } \\
\text { velocity }\end{array}$ \\
\hline [220] & $\begin{array}{l}\text { Carbon/glass reinforced } \\
\text { polyphenylene } \\
\text { sulphide/polyethylene } \\
\text { matrix }\end{array}$ & 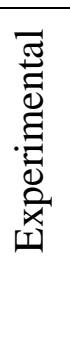 & ANN & $\begin{array}{c}\text { Material } \\
\text { composition, number } \\
\text { of matrix and } \\
\text { reinforcement layers, } \\
\text { manufacturing } \\
\text { process }\end{array}$ & $\begin{array}{l}\text { Mechanical } \\
\text { properties }\end{array}$ \\
\hline
\end{tabular}




\begin{tabular}{|c|c|c|c|c|c|}
\hline [221] & $\begin{array}{l}\text { Glass reinforced } \\
\text { polypropylene } \\
\text { composites }\end{array}$ & 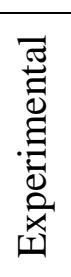 & ANN & $\begin{array}{l}\text { Fiber orientation } \\
\text { angle, cooling rate }\end{array}$ & $\begin{array}{c}\text { Stress-strain } \\
\text { behavior }\end{array}$ \\
\hline [222] & $\begin{array}{l}\text { Fiber Reinforced } \\
\text { Polymer (FRP) } \\
\text { composite }\end{array}$ & 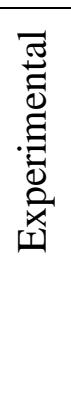 & ANN & $\begin{array}{c}\text { FRP thickness, } \\
\text { elastic modulus, } \\
\text { concrete } \\
\text { compressive } \\
\text { strength, aggregate } \\
\text { size, adhesive tensile } \\
\text { strength }\end{array}$ & $\begin{array}{l}\text { FRP-concrete } \\
\text { bond strength }\end{array}$ \\
\hline [223] & $\begin{array}{l}\text { Cotton fiber reinforced } \\
\text { polypropylene } \\
\text { composite }\end{array}$ & 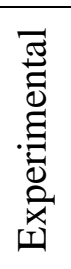 & ANN & $\begin{array}{l}\text { Mechanical } \\
\text { properties }\end{array}$ & $\begin{array}{l}\text { Optimal filler } \\
\text { content }\end{array}$ \\
\hline [224] & $\begin{array}{l}\text { CNT/fly ash/coir } \\
\text { reinforced epoxy } \\
\text { composites }\end{array}$ & 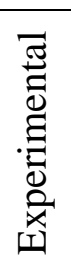 & ANN & $\begin{array}{l}\text { Weight fraction of } \\
\text { CNT, fly ash and } \\
\text { coir in the composite }\end{array}$ & Shear modulus \\
\hline [225] & $\begin{array}{l}\text { GFRP composite } \\
\text { laminates }\end{array}$ & 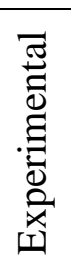 & $\begin{array}{l}\text { ANN + GA } \\
\text { (Genetic } \\
\text { Algorithm) }\end{array}$ & $\begin{array}{l}\text { Laser drilling } \\
\text { parameters }\end{array}$ & $\begin{array}{l}\text { Width of heat } \\
\text { affected zone, } \\
\text { bearing strength }\end{array}$ \\
\hline [226] & $\begin{array}{l}\text { GFRP composite } \\
\text { laminates }\end{array}$ & 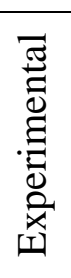 & ANN & $\begin{array}{c}\text { Slurry pressure, } \\
\text { nozzle diameter, } \\
\text { angle of } \\
\text { impingement }\end{array}$ & Erosion loss \\
\hline [227] & $\begin{array}{l}\text { Multi-layered polymer } \\
\text { composites }\end{array}$ & 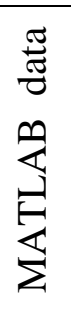 & ANN & $\begin{array}{l}\text { Number of layers, } \\
\text { angle of orientation }\end{array}$ & $\begin{array}{l}\text { Eigen values of } \\
\text { the } A B D \text { matrix }\end{array}$ \\
\hline
\end{tabular}




\begin{tabular}{|c|c|c|c|c|c|}
\hline [228] & $\begin{array}{l}\text { Carbon fiber reinforced } \\
\text { polymer composites }\end{array}$ & 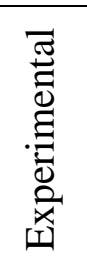 & ANN & $\begin{array}{l}\text { Amplitude damage } \\
\text { index, phase damage } \\
\text { index }\end{array}$ & $\begin{array}{c}\text { Quantification } \\
\text { of damage }\end{array}$ \\
\hline [229] & $\begin{array}{l}\text { Banana/coir fiber } \\
\text { reinforced polymer } \\
\text { composite }\end{array}$ & 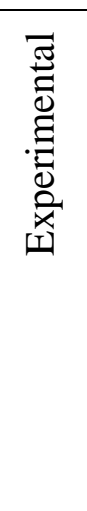 & ANN & $\begin{array}{l}\text { Weight fraction of } \\
\text { banana and coir, } \\
\text { percentage of alkali } \\
\text { treatment, } \\
\text { temperature and } \\
\text { pressure during the } \\
\text { process of } \\
\text { compression } \\
\text { moulding }\end{array}$ & $\begin{array}{l}\text { Grey relational } \\
\text { grade }\end{array}$ \\
\hline [230] & $\begin{array}{c}\text { Graphene } \\
\text { nanoplatelet/polylactic } \\
\text { acid nanocomposite }\end{array}$ & 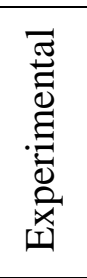 & ANN & $\begin{array}{c}\text { Process parameters } \\
\text { of plasma sintering } \\
\text { technique }\end{array}$ & $\begin{array}{c}\text { Density and } \\
\text { hardness }\end{array}$ \\
\hline [231] & $\begin{array}{l}\text { Basalt/glass reinforced } \\
\text { epoxy composites }\end{array}$ & 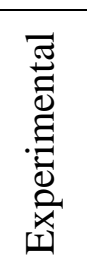 & ANN & $\begin{array}{c}\text { Lamp current, pulse } \\
\text { frequency, air } \\
\text { pressure }\end{array}$ & $\begin{array}{c}\text { Hole circularity } \\
\text { heat affected } \\
\text { zone }\end{array}$ \\
\hline [232] & $\begin{array}{l}\text { Vinyl ester composites } \\
\text { reinforced with nano } \\
\text { graphite particles }\end{array}$ & 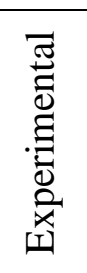 & ANN & $\begin{array}{l}\text { Filler volume } \\
\text { fraction, loading } \\
\text { frequency, } \\
\text { temperature }\end{array}$ & $\begin{array}{l}\text { Storage and } \\
\text { loss modulus }\end{array}$ \\
\hline [233] & $\begin{array}{l}\text { Unidirectional silica } \\
\text { reinforced polymer } \\
\text { composites }\end{array}$ & 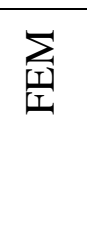 & ANN & Strain components & $\begin{array}{c}\text { Stress } \\
\text { components, } \\
\text { damage } \\
\text { information }\end{array}$ \\
\hline [234] & $\begin{array}{c}\text { Glass/basalt/carbon } \\
\text { reinforced polymer } \\
\text { sheets }\end{array}$ & 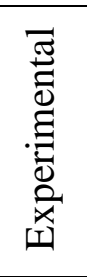 & ANN & $\begin{array}{c}\text { Density, thickness, } \\
\text { elastic modulus, } \\
\text { Poisson's ratio }\end{array}$ & $\begin{array}{l}\text { Bending } \\
\text { behavior }\end{array}$ \\
\hline [235] & $\begin{array}{l}\text { Short E-glass reinforced } \\
\text { polymer composite }\end{array}$ & $\sum_{\substack{\mid=1 \\
I}}$ & ANN & Strain components & $\begin{array}{c}\text { Stress } \\
\text { components }\end{array}$ \\
\hline
\end{tabular}




\begin{tabular}{|c|c|c|c|c|c|}
\hline [236] & $\begin{array}{l}\text { Compression moulded } \\
\text { glass fiber reinforced } \\
\text { polyester composites }\end{array}$ & 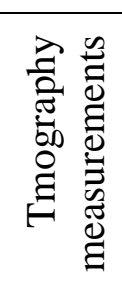 & ANN & $\begin{array}{l}\text { Initial charge size, } \\
\text { thickness of the } \\
\text { sample }\end{array}$ & $\begin{array}{c}\text { Fiber } \\
\text { orientation }\end{array}$ \\
\hline [237] & $\begin{array}{c}\text { Silica reinforced } \\
\text { polyvinyl pyrrolidone } \\
\text { composite }\end{array}$ & 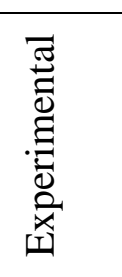 & ANN & Mass, frequency & $\begin{array}{c}\text { Coefficient of } \\
\text { sound } \\
\text { absorption of } \\
\text { the material }\end{array}$ \\
\hline [238] & $\begin{array}{l}\text { Short glass fiber } \\
\text { reinforced epoxy } \\
\text { composite }\end{array}$ & 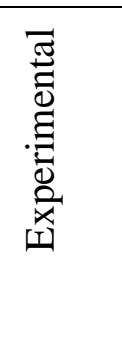 & ANN-GA & $\begin{array}{c}\text { Mold temperature, } \\
\text { melt temperature, } \\
\text { water temperature, } \\
\text { water pressure, delay } \\
\text { time }\end{array}$ & $\begin{array}{l}\text { Standard } \\
\text { deviation } \\
\text { depicting } \\
\text { residual wall } \\
\text { thickness } \\
\text { uniformity }\end{array}$ \\
\hline [239] & $\begin{array}{l}\text { Graphene/boron carbide } \\
\text { particles reinforced } \\
\text { polycarbonate } \\
\text { composites }\end{array}$ & 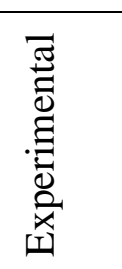 & ANN & $\begin{array}{c}\text { Material } \\
\text { composition and } \\
\text { tribo-test parameters }\end{array}$ & $\begin{array}{l}\text { Wear rate, } \\
\text { coefficient of } \\
\text { friction }\end{array}$ \\
\hline [240] & $\begin{array}{l}\text { Hybrid composites of } \\
\text { epoxy reinforced with } \\
\text { short human hair and } \\
\text { glass microspheres }\end{array}$ & 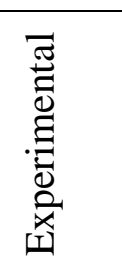 & ANN & $\begin{array}{l}\text { Process parameters } \\
\text { of pin-on-disc test }\end{array}$ & Wear rate \\
\hline [241] & $\begin{array}{l}\text { Polyvinylchloride } \\
\text { composites }\end{array}$ & 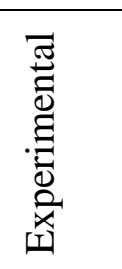 & ANN & $\begin{array}{c}\text { Particle size of } \\
\text { various fillers used }\end{array}$ & $\begin{array}{c}\text { Ductility, } \\
\text { density, tensile } \\
\text { strength }\end{array}$ \\
\hline [242] & $\begin{array}{l}\text { Natural fiber reinforced } \\
\text { epoxy composites }\end{array}$ & 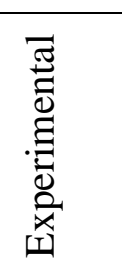 & $\begin{array}{c}\text { Recurrent } \\
\text { neural network }\end{array}$ & $\begin{array}{l}\text { Process parameters } \\
\text { of acoustic emission }\end{array}$ & $\begin{array}{l}\text { Cutting } \\
\text { conditions }\end{array}$ \\
\hline [243] & $\begin{array}{c}\text { CNT reinforced } \\
\text { polymer composites }\end{array}$ & 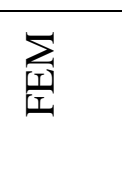 & ANN & $\begin{array}{c}\text { Geometry, } \\
\text { conductivity, weight } \\
\text { fraction of } \mathrm{CNT}\end{array}$ & $\begin{array}{c}\text { Bulk } \\
\text { conductivity }\end{array}$ \\
\hline
\end{tabular}




\begin{tabular}{|c|c|c|c|c|c|}
\hline [244] & $\begin{array}{c}\text { Polymer } \\
\text { nanocomposites }\end{array}$ & $\sum_{\substack{\mid I \\
I}}$ & $\mathrm{CNN}$ & $\begin{array}{l}\text { 2D microstructural } \\
\text { images }\end{array}$ & $\begin{array}{l}\text { Viscoelastic } \\
\text { properties }\end{array}$ \\
\hline [245] & $\begin{array}{l}\text { Graphene reinforced } \\
\text { polymer } \\
\text { nanocomposites }\end{array}$ & $\sum_{\mid=1}^{i}$ & ANN & Electric field vector & Current density \\
\hline [246] & $\begin{array}{l}\text { Cotton fiber/fly ash } \\
\text { reinforced polyester } \\
\text { composites }\end{array}$ & 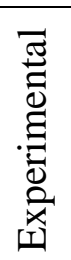 & ANN & $\begin{array}{l}\text { Material parameters, } \\
\text { pin-on-disc test } \\
\text { conditions }\end{array}$ & Wear rate \\
\hline [247] & $\begin{array}{l}\text { Glass/carbon reinforced } \\
\text { epoxy composites }\end{array}$ & 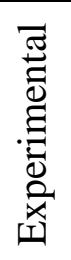 & ANN & $\begin{array}{l}\text { Sliding distance, } \\
\text { speed, load }\end{array}$ & $\begin{array}{l}\text { Mass loss, } \\
\text { coefficient of } \\
\text { friction }\end{array}$ \\
\hline [248] & $\begin{array}{l}\text { Polypyrrole coated } \\
\text { CNT reinforced } \\
\text { nanocomposites }\end{array}$ & 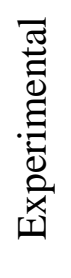 & ANN & $\begin{array}{l}\text { Membrane pressure, } \\
\text { temperature, time, } \\
\text { concentration of } \\
\text { polypyrrole and } \\
\text { CNT }\end{array}$ & $\begin{array}{c}\text { Flux } \\
\text { measurements }\end{array}$ \\
\hline [249] & $\begin{array}{l}\text { Carbon fiber reinforced } \\
\text { polymer composite }\end{array}$ & 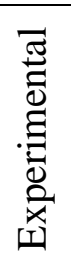 & $\begin{array}{c}\text { Back } \\
\text { propagation } \\
\text { neural } \\
\text { network-GA }\end{array}$ & $\begin{array}{l}\text { Cutting speed, speed } \\
\text { of the spindle, depth } \\
\text { of the cut }\end{array}$ & $\begin{array}{l}\text { Cutting force } \\
\text { and } \\
\text { delamination }\end{array}$ \\
\hline [250] & $\begin{array}{c}\text { Carbon } \\
\text { black/styrene/butylacryl } \\
\text { ate composites }\end{array}$ & 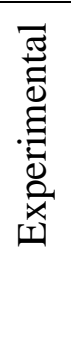 & ANN & $\begin{array}{l}\text { Quantity of carbon } \\
\text { black, monomer } \\
\text { ratio, content of } \\
\text { surfactant }\end{array}$ & $\begin{array}{l}\text { Glass transition } \\
\text { temperature, } \\
\text { Particle size, } \\
\text { Colorimetric } \\
\text { and thermal } \\
\text { properties }\end{array}$ \\
\hline [251] & $\begin{array}{c}\text { Yarn reinforced } \\
\text { polymer composites }\end{array}$ & 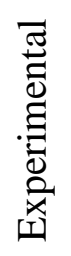 & $\begin{array}{c}\text { Back } \\
\text { propagation } \\
\text { neural network }\end{array}$ & Temperature & $\begin{array}{l}\text { Thermogravime } \\
\text { tric parameters }\end{array}$ \\
\hline
\end{tabular}




\begin{tabular}{|c|c|c|c|c|c|}
\hline [252] & $\begin{array}{l}\text { Carbon fiber reinforced } \\
\text { bismaleimide } \\
\text { composites }\end{array}$ & 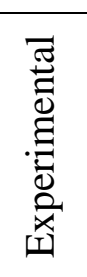 & $\mathrm{CNN}$ & Heating pattern & $\begin{array}{c}\text { Features of } \\
\text { microwave } \\
\text { control strategy }\end{array}$ \\
\hline [253] & $\begin{array}{l}\text { Wood fiber reinforced } \\
\text { thermoplastic starch } \\
\text { composites }\end{array}$ & 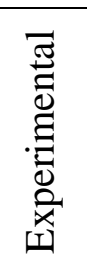 & $\begin{array}{c}\text { Back } \\
\text { propagation } \\
\text { neural network }\end{array}$ & $\begin{array}{l}\text { Content of } \\
\text { plasticizer, cross- } \\
\text { linking agent, } \\
\text { blowing agent }\end{array}$ & Tensile strength \\
\hline [254] & $\begin{array}{l}\text { Ionic polymer-copper } \\
\text { composites }\end{array}$ & 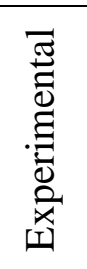 & $\begin{array}{c}\text { Back } \\
\text { propagation } \\
\text { neural network }\end{array}$ & $\begin{array}{l}\text { Dimension ratio, } \\
\text { simulation voltage }\end{array}$ & $\begin{array}{l}\text { Displacement, } \\
\text { blocking force }\end{array}$ \\
\hline$[255]$ & $\begin{array}{l}\text { Bisphenol epoxy } \\
\text { samples }\end{array}$ & 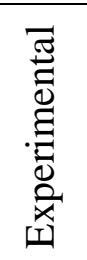 & ANN & $\begin{array}{l}\text { Ultimate tensile } \\
\text { strength }\end{array}$ & $\begin{array}{c}\text { Residual } \\
\text { strength, } \\
\text { exposure time, } \\
\text { temperature }\end{array}$ \\
\hline [256] & $\begin{array}{l}\text { Carbon fiber reinforced } \\
\text { polymer composites }\end{array}$ & 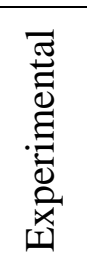 & $\begin{array}{c}\text { Back } \\
\text { propagation } \\
\text { artificial neural } \\
\text { network }\end{array}$ & $\begin{array}{l}\text { Feeding speed, depth } \\
\text { of cut, spindle speed }\end{array}$ & $\begin{array}{l}\text { Cutting force, } \\
\text { delamination } \\
\text { factor }\end{array}$ \\
\hline [257] & $\begin{array}{l}\text { Carbon fiber reinforced } \\
\text { epoxy composites }\end{array}$ & 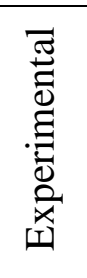 & ANN & $\begin{array}{c}\text { Humidity, } \\
\text { temperature, } \\
\text { emissivity, Distance } \\
\text { between the source } \\
\text { and the specimen }\end{array}$ & $\begin{array}{c}\text { Depth of the } \\
\text { defect }\end{array}$ \\
\hline [258] & $\begin{array}{l}\text { Short glass fiber/slag } \\
\text { reinforced } \\
\text { polypropylene } \\
\text { composites }\end{array}$ & 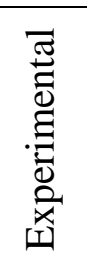 & ANN & $\begin{array}{l}\text { Velocity of impact, } \\
\text { impingement angle, } \\
\text { filler content, size } \\
\text { and temperature of } \\
\text { erodent }\end{array}$ & $\begin{array}{l}\text { Erosion wear } \\
\text { rate }\end{array}$ \\
\hline [259] & $\begin{array}{l}\text { Carbon fiber reinforced } \\
\text { polymer composites }\end{array}$ & $\sum_{\substack{\mid=1 \\
I}}$ & ANN & $\begin{array}{l}\text { Orthotropy ratio of } \\
\text { the stiffened panel } \\
\text { and the plate, } \\
\text { young's modulus, } \\
\text { stiffness coefficients }\end{array}$ & $\begin{array}{c}\text { Shear load that } \\
\text { can cause } \\
\text { buckling }\end{array}$ \\
\hline
\end{tabular}


accurately [198]. Different variants of neural networks have been successfully used by material scientists to predict their behavior under different conditions and some more of such related studies are presented in Table 1. In view of the effectiveness and several successful attempts, while it is worth considering the adoption of neural networks in the analyses of polymer composites, we present a critical review of other prominent machine learning techniques in the following subsections.

\subsection{Linear and Logistic regression}

Linear regression is a simple, old, and extensively used technique to make predictions when the target variable is continuous (for regression problems). It predicts the output variable based on a straight fit line and the prediction function is of the following form

$$
Y=W \times X+B
$$

It considers that the input $(X)$ and output variables $(Y)$ are linearly related to each other [260]. W represents the weight and $B$ represents the bias which is the intercept used to offset the predictions. Weight and bias are the variables that the algorithm learns in order to make the most accurate predictions. It may be noted in this context that it is possible to bring in some degree of non-linearity between $X$ and $Y$ by appropriately using transformations (such as power, logarithmic, exponential etc.) of the input and output variable sets and thereby using linear regression as per equation (4) based on the transformed variables. Mean square error is generally used to quantify the goodness of fit between the actual and the predicted values. On the other hand, logistic regression is generally used for predicting discrete values where the target variable is categorical (for classification problems) and is commonly known as the classification algorithm of machine learning. This algorithm of supervised learning involves adding a threshold value and using activation functions in order to convert linear equation to logistic regression equation and also, to map the available values between 0 and 1 . In this case, probabilistic predictions are made by fitting a curve to the given data [261]. For this purpose, a sigmoid function of the following form is commonly used

$$
Y=\left(1+e^{-x}\right)^{-1}
$$

Considering the simplicity of this ML algorithm, it has been extensively used for prediction and classification in a multitude of problems [262-264]. Sakaguchi et al. [265] explained the correlation between the polymerization contraction force and the energy density with the help of linear regression. Noryani et al. [266] used various statistical models for the material selection of natural fiber reinforced polymer composites. Based on the correlation coefficient, analysis of variance and the R-squared value, linear regression model was chosen for this purpose. Another group [267] developed a conductive 
composite material made of polyurethane filled with carbon black, wherein they studied the response of this composite material at a particular temperature and vapor pressure. A linear regression model was used to explain the relationship between these two parameters and the response rate of the composite. Kleverlaan et al. [268] investigated the shrinkage stress developed in resin composites concerning the field of dentistry. They used the framework of linear regression to establish a correlation between the shrinkage and the contraction stress and, between the shrinkage stress and the tensile modulus. They found out that the amount of shrinkage/contraction stress and the tensile modulus developed in the material largely depends on the content of resin which is not polymerized. Gu et al. [269] applied logistic regression to predict the performance of the polymer composites having different combinations of geometry and material at a less computational cost. $\mathrm{Xu}$ et al. [270] made use of the logistic regression model to identify various damage modes in adhesive joints of fiber-reinforced polymer composites. They found that different damage modes viz. adhesive failure, delamination, fiber breakage, matrix/fiber separation from adhesive were all the subspace of the used acoustic emission parameters. The main application of logistic regression method has been found in classification problems e.g. for identifying the key parameters affecting the performance of a material using the given structural signals and for classification of defects and damage in composite materials [158,159]. Cao et al. [273] used the algorithm of logistic regression to assess the stability of different polymers based on the thermogravimetric analyses. Folorunso et al. [274] used the approach of logistic regression for the parametric analysis of electrical conductivity of different polymer composites. Polymer based materials are often used as dental filling materials. But during the initial hours of curing, a few substances release from the filling and it might have harmful effects. Berge et al. [275] applied the framework of logistic regression to investigate the effect of those substances on the health of a foetus. Osburg et al. [276] used logistic regression to classify the consumer interest for buying wood-based polymer composites. Logistic regression has been successfully used to investigate the thermal response of polymer composites by multiple researchers [277,278].

\subsection{Gaussian process (GP) and kriging}

Gaussian process (GP) is a non-parametric stochastic process which is a powerful tool for solving non-linear problems [279]. To bridge the gap between computer simulations and physical conditions, Gaussian process is one of the Naïve Bayer's variants that is widely used [280]. This method is most commonly used in geostatistics which deals with modeling the spatial aspects of the world. GP has found a huge acceptance in various fields like oceanography, finance and physics [281285]. With the increasing complexity of the datasets, simple parametric approaches tend to lack in 
accuracy and effectiveness. Implementation of neural networks also becomes tricky in such situations [286]. But the development of kernel methods like Support Vector Machines (SVM) and GP has made the inferences and predictions quite flexible and effective even if the data available for the training purpose is small. Gaussian process, in a nutshell, is a group of random variables with an assumption that all the dependent and independent variables have common Gaussian distribution [287]. A Gaussian process is characterized by two functions namely its mean, $\mu\left(x_{i}\right)$, and the covariance, $c\left(x_{i}, x_{j}\right)$, where $i, j$ varies from 1 to $n$. The parameter $n$ is the number of data points and $x$ is the input vector. Expression for a basic GP model is given by

$$
F(x)=G P\left(\mu\left(x_{i}\right), c\left(x_{i}, x_{j}\right)\right)
$$

The final output is expressed as

$$
Y(x)=F(x)+\operatorname{err}(x)
$$

where $Y(x)$ is the output of interest and $\operatorname{err}(x)$ is the error associated with the noise in the dataset. GP does not require setting a hypothesis and finding appropriate values for the weights in the framework, rather it produces a distribution of all the possible functions which are somehow consistent with the training data. Kernel functions used in GP produce a covariance matrix which is Gaussian distributed and the final GP model makes use of it whenever an output is required to be predicted for a new unseen dataset [288]. Gaussian process involves boundless parameters that keep growing with the training data [289,290]. GP is mostly used to model the probability of such data in which the variables/features are of continuous nature [291].

Considering the efficacy of the method, many researchers have used GP for predicting material behavior of polymer composites. Liu et al. [292] presented a prognostic framework based on Gaussian process in order to predict the remaining life and states of damage in polymer composites. Acoustic and piezoelectric parameters were used as the dependent and independent variable space of the used GP. A good correlation was found between the experimental and predicted results. Wertz et al. [293] developed a surrogate model considering the ultrasonic response of composite structures when subjected to delamination at different locations. They obtained the B-scans of the composites and applied a Gaussian process regression model on the parameters of chirplet transform. GP reduced the computational time by $95 \%$ and made it possible to characterize the impact damage of composite laminates with reasonable accuracy. Gaussian process regression was found to be quite robust and efficient for fitting non-linear topologies and smaller feature space. Wang et al. [294] investigated the interphase properties of polymer nanocomposites by making use of adaptive optimization. They used 
Gaussian process to correlate the feature space with the interphase properties in terms of its viscoelastic and dielectric behavior. GP was chosen to be used as a surrogate model due to its ability of considering uncertainties and assessing the non-linear response with minimum random error. Another research group [295] used GP to investigate the effectiveness of using surface response to excitation method for structural health monitoring. They used a plate made up of carbon fiber reinforced epoxy composite and attached piezoelectric elements to it. Load was applied on the plate and the transmitted waves were recorded at certain locations. Using characteristic transfer functions, dataset for Gaussian process regression was obtained and accurate predictions for the location of load were made. Hansoge et al. [296] applied Gaussian process to predict the mechanical behavior of hairy nanoparticles reinforced polymer composites. Bond strength between the polymer and the nanoparticles, grafting density, chain length and the edge length of the nanoparticle were used as the input parameters, while toughness modulus of the resulting composite was the output variable. Training data was obtained from molecular dynamics simulations and then the Gaussian process regression was performed. The results obtained from GP and the molecular dynamics simulations were in good agreement with each other. Qin et al. [297] studied the effective permittivity of microwire polymer composites within 1-6 GHz frequency range. Effect of increasing the concentration of wire on the strain sensitivity and permittivity was studied using a GP model. Gaussian processes can also be used for analyzing the microstructural images of composites to extract important information from them. Gaussian filters have been used for reducing the noise in the image as well as in the signal [298]. Schadler et al. [299] developed a design methodology for polymer based nano-dielectrics to make predictions for their breakdown strength. Finite element modeling and Monte Carlo simulations were performed for the loss functions and the dielectric constant. Results obtained from these simulations were then meta-modeled using Gaussian process to study the effect of loading and filler dispersion on the breakdown strength, loss functions and the dielectric constant. Li et al. [300] investigated the adhesive properties of polymer composite laminates to understand the debonding behavior. They used adhesives of different properties and followed Gaussian process to predict the toughness and bonding strength. It was found that the spatial heterogeneity of different adhesives affects the crack resistance of joints and the adhesive failure strain is dependent on the ligament elongation and the dissipated energy.

Another surrogate model based on the Gaussian process that is considered cost-effective and compact for complex computations is kriging. Kriging uses the idea of interpolation directed by prior covariances in order to obtain the responses corresponding to the unseen data. The unknown response in this method is represented as

$$
\hat{g}(X)=y_{0}(X)+Z(X)
$$


where $\hat{g}(X)$ is the unknown function of interest, $X$ is an $n$ dimensional vector ( $n$ is number of design variables), $y_{0}(X)$ is the known approximation function which is usually a polynomial and $Z(X)$ represents the realization of a stochastic process with zero mean and variance along with nonzero covariance. A hybrid approach of kriging and ML covariates has been used to make successful spatial interpolations in environmental sciences. Neural networks alone cannot have as accurate results as specific geostatistical tools, but using the outputs of neural networks as covariates in a kriging model produces highly accurate results for geospatial analyses [301]. Kriging has been successfully used to classify and cluster data with accuracy [302]. Considering the simplicity and reduced computational time of Gaussian process and kriging, in recent years this methodology has been used extensively to model the physical properties of different polymer composites with fairly good accuracy [303-317]. Lately, Mukhopadhyay et al. [106] investigated the performance of various kriging model variants (such as ordinary kriging, universal kriging based on pseudo-likelihood estimator, blind kriging, cokriging and universal kriging) to study the dynamic behavior of polymer composite laminates. It was revealed that universal kriging coupled with marginal likelihood estimate obtains the most accurate results, followed by co-kriging and blind kriging. According to computational efficiency, it was observed that for high-dimensional problems, the CPU time required for constructing co-kriging model is significantly less compared to the other kriging model variants.

\subsection{Support vector machines (SVM)}

Support Vector Machines (SVM) is an algorithm of supervised machine learning that can be used to solve classification and regression problems [318]. When it comes to solving the classification problems comprising of huge data, SVM has always been considered as the most classic technique under the broad domain of machine learning [319,320]. In SVM, each data point is plotted in $x$ dimensional space, where $x$ is the total number of features available. Here the goal is to find a hyperplane that classifies/splits all the training vectors in different classes. Hyperplane is always a ( $x$ 1) dimensional separator [321]. SVM is based on the idea of optimization, where the hyperplane is chosen in a way such that the distance between the support vectors (data points) and the data separator has the widest possible margin [322]. Support vector machines are versatile in their application as they make use of kernelization that allows the algorithm to model the non-linear decision boundaries by adding another dimension to the given data [323]. The predictions are made based on the mapping of each input feature to a transformed basis vector. A special version of support vector machine is support vector regression (SVR) which is particularly developed for estimating real value functions. In order to construct a model having maximum deviation of $\varepsilon$ from the function value of every training point, it 
uses a data subset which is known as the support vectors. In case of linear regression, this model can be mathematically written as

$$
\hat{g}(X)=\langle W . X\rangle+B
$$

where $\hat{g}(X)$ is the approximate value of the objective function at $X$. The parameter $W$ represents a vector of weights, $B$ is the bias, and $\langle$.$\rangle denotes the inner product.$

The SVM based machine learning models have been widely adopted in the analysis of polymer composites. Datta et al. [324] used support vector regression to predict the location of impact and quantify the energy of this event in the case of CFRP composites. Khan et al. [318] developed a structure-property relationship model for correlating the dynamic properties of polymer nanocomposites with filler percentage and temperature using the algorithm of support vector regression. Zhang et al. [325] used the method of support vector regression to evaluate the bond strength of fiber-reinforced polymer with concrete. They claimed that this algorithm can be conveniently applied to solve other regression problems existing in the domain of structural engineering. Another research group [326] came up with a prediction and optimization model based on support vector regression for designing materials based on the conductivity of CFRP composites. Mardanshahi et al. [327] used support vector machines to detect and classify matrix cracking in laminates of glass fiber reinforced epoxy composites. They induced matrix cracking of varying intensity in all the composite laminates and used lamb wave propagation for non-destructive testing. The signals extracted from the experimental study were processed and used as the training dataset for constructing SVM network, where a classification accuracy of 91.7\% was found. Meng et al. [328] used carbon fiber reinforced polymer composites with preexisting defects like delamination and voids to understand the classification of ultrasonic signals from the composite specimens. They proposed the utilization of support vector machine in conjunction with convolutional neural networks and classified the defects at various locations. Performance of the proposed framework was validated with experimental results. Another research group [329] used three different kernel functions to classify the damage caused in composite samples due to an impingement ranging from 5 to $7 \mathrm{~mm}$. The basic framework of SVM was used to test the performance of every input feature. Results indicated that SVM can be effectively used for image based analysis to quantify damage in composite laminates. Abuomar et al. [330] used support vector machine to classify the mechanical properties of vinyl ester nanocomposites reinforced with vapor grown carbon fibers. The entire dataset was classified into ten classes and 3-folds cross validation was used. In order to assess the performance of SVM classifier, confusion matrices were used in different sets. This method was proven to be a very quick and reliable 
technique for classifying large datasets. Surface damage is unavoidable during the machining operation of polymer composites. Delamination is the most common form of damage that can be observed during drilling of fiber reinforced polymer composites and it becomes the primary reason for material failure as the overall strength is reduced. In this view, Aich et al. [331] made an attempt to use a machine learning algorithm to accurately predict the delamination factor. They exploited support vector machine to investigate the underlying effect of each input variable on overall responses of the material, which further helped in modeling the delamination factor. $\mathrm{Xu}$ et al. [332] used SVM in combination with

algorithms of particle-swarm optimization to predict the degree of damage in cables made up of carbon fiber reinforced polymer composites. The particle swarm algorithm made use of Acoustic Emission (AE) signals and later trained the SVM with AE parameters. This paper demonstrated the feasibility of using AE with ML algorithm to judge the extent of damage in the composites. Another research group [333] performed nano indentation testing on CFRP composites to characterize their structural integrity. They used SVM and K-nearest neighbors for identifying the reinforcement and assessing its quality. A correlation between the interphase properties of the composite and its reinforcement was also established. Altarazi et al. [334] fabricated polymer composites using two different techniques namely, compression moulding and extrusion blow moulding. They used nine ML algorithms to predict the flexure strength of the composites as a function of material composition and manufacturing conditions. Out of these nine ML models, support vector machine showed the best results with maximum accuracy. Dey et al. [335] investigated the effect of cutout on stochastic dynamics of composite laminates using a SVM assisted finite element approach, where a high level of computational efficiency was established. For tasks like damage/defect detection, risk factor identification, estimation of impact severity and prediction of natural hazards, SVM has proven its efficiency by giving superior results [321-325].

\subsection{Ensemble based methods}

Ensemble methods are a combination of various base algorithms which result in a predictive model that outperforms each one of the individual models. Integrating various ML models in order to obtain an intelligent global predictive system results in reliable decisions with least generalization error [341]. The main idea is to weigh various individual models and accordingly combine them to produce the most optimal one [342]. The idea of this methodology under the domain of supervised learning came into existence in the late seventies and since then researchers started exploring it [343]. The first ensemble method was used by combining two linear regression models and nearest neighbor method to enhance the performance of recognition systems [344]. To determine the target variable, decision trees are very often used as ensemble method [345,346]. 
Multiple applications of such ensemble based ML methods can be traced in the area of polymer composites. Liu et al. [347] used an ensemble based methodology to predict fields of elastic deformation in the polymer composites. Different regression models were combined together to make accurate predictions. Dataset used for the training purpose was obtained from 3D microstructural images of the composites. In order to compare the advantages of additive manufacturing over the conventional method, Zhang et al. [348] used ensemble based machine learning model to predict the flexural strength of CFRP composites and compared the results with a linear regression and Physics based model. Ensemble based methodology achieved the best accuracy along with establishing an efficient relationship among the number of fiber layers, its orientation and matrix infill patterns. Another research group [349] also adopted this methodology to analyze the thermographic data of CFRP composites for classifying the surface defects. Gaudenzi et al. [350] used an experimental procedure to classify the damage caused in CFRP composite laminates. They used low velocity impact to induce delamination in the composites and then applied wavelet packet transform to obtain damage features from the dynamic response of affected and non-affected specimens. Subsequently, those features were classified using ensemble based methods. In general, the ensemble based methods are extensively used in the area of structural health monitoring and damage assessment of composite structures [351,352]. Zang et al. [353] characterized natural fiber reinforced polymer composites for their surface finish using acoustic emission sensors. The complex heterogeneous structure of these composites results in adverse effects on the integrity of the machined surface. Therefore, to understand the time-frequency relationships of acoustic emission signals and their correlation with the material cutting mechanism, fiber orientation and speed of cutting during the machining process, ensemble based method was exploited. Pathan et al. [354] made use of Gradient Boosted tree Regressor (GBR) to predict the mechanical response of unidirectional fiber reinforced polymer composites based on their microstructural images. This GBR is an ensemble based technique whose performance depends on the number of estimators, learning rate and the depth of decision tree. Guo et al. [355] applied ensemble based learning to polyimide nanocomposites in the form of gradient boosting so as to predict their breakdown field strength. They prepared 32 composite samples varying in thickness and material composition. Voltage test equipment was used to measure the strength experimentally. Their prediction was reported to be accurate. From the concise literature survey presented in this subsection, it may be noted that the nature of considering a myriad of ML algorithms and then integrating these algorithms together to produce the optimal one is what makes ensemble techniques stand out [356]. 


\section{Accuracy checking criteria}

The most crucial step in developing any predictive model is evaluating its performance. It is very important to evaluate the prediction accuracy of the ML model for unseen data. Based on the problem in hand whether its regression or classification, different performance evaluating metrics are used. Confusion matrix, accuracy, precision, recall, receiver operating characteristics curve are a few metrics commonly used for assessing the performance of a classification problem [357,358]. Confusion matrix is a $n \times n$ matrix that represents the actual and predicted results of a classification problem, where $n$ represents the number of classes taken into consideration [359]. Common terms used in a confusion matrix are: True Positives (TP), False Positives (FP), True Negatives (TN) and False Negatives (FN). True positives indicate the outcomes when predicted and the actual results are positive and false positives refer to the outcomes when the predicted results are negative while the actual ones are positive. True negatives refer to the positive predicted and negative actual values whereas false negatives refer to negative predicted and positive actual results. Outcomes of the confusion matrix are considered as a direct measure of the model's performance [360]. Accuracy is the second most common evaluation metric but doesn't give a reliable measure of performance when the data classes are not balanced. The precision is defined as the ratio of positive outcomes (when the predicted and actual results belong to the same class) to the total predicted instances (TP+FP). Recall metric is also known as sensitivity or rate of true positives and is the ratio of TP to the sum of TP and FN. To evaluate the performance of a binary classifier, Receiver Operating Characteristics (ROC) curve is used. For different threshold limits, the graph is plotted between the true positive rate and the false positive rate. Then the area under this curve is calculated and a higher value of area indicates better performance of the chosen model. Pham et al. [361] used the ROC curve to evaluate the generalizability and the goodness of fit of various ML algorithms by considering the trade-off between the sensitivity and the specificity. Dwivedi [362] considered six different ML algorithms for the prediction of heart disease and compared their performance on the basis of ROC curve, where the logistic regression was found to have the highest classification accuracy. Probability Density Function (PDF) plots are also utilized to statistically define the likelihood of a particular result and the data distribution. PDF plots give a measure of the expected value (mean of the random variables) and the variance. Classification rate is another accuracy measure used for various classifiers when the problem in hand has multiple classes [363]. The classification rate is determined as

$$
\text { Classification rate }=\frac{\text { No. of observations classified correctly }}{\text { Total no. of observations }}
$$


On the other hand, for regression problems Mean Absolute Error (MAE), Root Mean Square Error (RMSE) and coefficient of determination are frequently used for checking prediction accuracy. These metrics give an idea of how close/far the predicted values are from the actual ones.

$$
\begin{aligned}
M A E & =\frac{1}{n} \sum_{i=1}^{n} \frac{\left|P_{i}-A_{i}\right|}{A_{i}} \\
R M S E & =\sqrt{\frac{1}{n} \sum_{i=1}^{n}\left(P_{i}-A_{i}\right)^{2}}
\end{aligned}
$$

where $n$ indicates the number of observations, $A$ refers to the actual value and $P$ refers to the corresponding predicted value. Coefficient of determination is based on the variance of the model considering the dependent and the independent variables. It is commonly denoted as $R^{2}$ and its value always lies between 0 and 1, where a higher value indicates better accuracy. For judging the best correlation between various parameters involved, scatter plots and parallel coordinate plots are also used. These plots are very helpful in characterizing the performance accuracy of different ML algorithms with a certain confidence interval. Li et al. [364] performed a comparative study to see the effectiveness of both these plots for determining the performance accuracy and scatter plots were found to outperform the other metric. Scatter plots are considered as one of the basic tools used for quality control where point-wise cross verification of the prediction is possible. N.K. Ostadi [365] used artificial neural networks, support vector machines and radial basis function to model the roughness index of pavement. He used scatter plots and sensitivity analysis to compare the qualitative performance of the mentioned algorithms. Also, the generalization accuracy of these algorithms was tested using the quantitative evaluation metric, the mean square error. Radial basis function was found to be the best algorithm based on the combined results of qualitative and quantitative performance measures. Anomalies in the form of outliers in the data affect the prediction accuracy of every ML algorithm [366]. Quick visualization of the available data is very important in applied machine learning to ensure a fair amount of accuracy in the predictions. To identify the specific patterns and outliers in the data, box plots are used to visualize the distribution of available numeric attributes through quartiles [367]. The originality of the data can be well maintained using the method of box-plots for the detection of outliers as this method does not require any pre-processing of the data [368]. To assess the prediction accuracy of ML algorithms, Bootstrap method, which is a resampling technique is also used. This method involves the iterative resampling of a dataset with replacement. The advantage of using bootstrap method is that the resulting sample often has a Gaussian distribution and it is easy to 
determine the variation in the actual and predicted values in terms of standard error, variance and confidence interval [369]. In most cases of polymer composites, applying more than one of these metrics to check the performance of ML model is imperative for identifying anomalies and for making reliable predictions [111].

\section{Summary and perspective}

Machine learning uses a range of statistical, probabilistic and optimization techniques to comprehend a physical space and subsequently predict, analyze and identify. It offers the opportunity to study larger and complex polymer composite systems that are not currently amenable to characterization, design and optimization. This review paper is an attempt to benefit the material scientists and encourage them to exploit these competent algorithms in order to identify and develop new features and multi-functionalities which have been hitherto unexplored due to computational limitations. It is a perplexing task to understand the algorithms used for predictive modeling thoroughly. But on the basis of this extensive literature survey, a few critical remarks for different ML algorithms are given below.

- $\quad$ For the problems having too many input variables, which results in high dimensional input vector space, support vector machine works the best in most of the cases.

- For drawing inferences from noise inflicted data, decision tables and neural networks perform better for classification and regression problems respectively.

- Logistic regression is one of the simplest algorithms for dealing with non-linear problems.

- Gaussian process has shown good results when the problem involves a combination of continuous and discrete variables.

- Polynomial chaos expansion is found to be very effective in quantifying the unavoidable uncertainties of polymer composites.

- If there is a scope of designing the input samples, it is found that D-optimal design (DoE method), Sobol sequence and Latin hypercube sampling normally lead to faster convergence and outperform other sampling techniques.

- Superior performance of any ML algorithm over others is considered to be problem-specific. No single ML algorithm is perfect for all the problems. Rather, the performance of any algorithm (accuracy, stability and computational efficiency) depends largely on the structure and size of the available data, dimension of the input parameter space, complexity of the model etc. 
In recent years, machine learning has been adopted by numerous material scientists and engineers due to its striking success in different other fields. However, considering the widespread application of machine learning, it is important to address some existing problems that arise during the implementation of these algorithms to practical problems [370] with the complexity of modeling polymer composite materials, as highlighted in the following subsections.

\subsection{Curse of dimensionality}

As mentioned earlier, the success of any ML algorithm depends on the data which is fed into the network (i.e. the quality of learning). A decent predictive model always requires good and sufficient data to learn from. Usually, the data used for training purposes is either obtained from computational simulations or experiments. In order to produce viable results, data in sufficient quantity and in proper

dimensionality is required [371]. Most of the ML algorithms work fine with the input feature vector of low dimensionality (provided that the input-output relationship is not too nonlinear or complex), but the same algorithms may lose their credibility when the dimensionality of the input space increases. This can be attributed to the fact that with increasing dimensionality, data sparsity increases and the generalization capability of the ML model reduces. To avoid the problems associated with it, a few dimensionality reducing techniques like Principal Component Analysis (PCA), multicollinearity, regularization, sensitivity analysis, feature ranking, variance filter and Independent Component Analysis (ICA) are used. Cacciola et al. [372] used the framework of support vector machine to classify manufacturing defects in CFRP plies. They used the technique of regularization to mitigate the curse of dimensionality. Xu et al. [373] used a ML based clustering method and acoustic emission technique to identify various types of damage modes in polymer composite joints. Principal component analysis was used to take out the redundant features and consequently avoid the problems associated with higher dimensional input space. It is important to select the input features appropriately so that a practical relationship could be established between the dependent and independent variables [374].

\subsection{Overfitting}

Overfitting is a challenge often faced by data scientists when the data in hand and the available knowledge are not sufficient to determine the objective function correctly. In such cases, the model tends to make random predictions which are not coherent with the actual results [375]. This might result in causing illusions to the user that they have attained a good accuracy in the training phase but actually the predictions are so random that the accuracy of the testing phase may be as less as 40-50\%. It can be better understood by considering the bias-variance tradeoff. Bias is the tendency of the model to learn consistently on the basis of wrong assumptions and keep learning the same wrong data, while 
variance is the error generated due to the model's sensitivity towards small fluctuations. Variance makes the model learn random noise regardless of the correct signal which results in random outputs rather than the intended ones. Feeding the network with too many or too less attributes/features is the main reason that causes overfitting or underfitting and both of these result in poor predictions [376]. Cross-validation is one of the possible solutions to combat this problem. Another solution is to penalize the model by adding a regularization term in the function which will be used for the evaluation purpose [377]. Feature selection is one of the best solutions to avoid overfitting and increase the efficiency of any machine learning algorithm. Data to be used for the training purpose ensuring that only the relevant and useful features are used [378,379]. There are methods like information gain, chi-square and correlation coefficient which are used to measure the individual effect of a feature on the target variable and then accordingly include selected features for the training purpose [380-382]. These methods are known as filter methods. There are wrapper and embedded techniques that help in overcoming the problem of overfitting [383,384]. Wrapper methods involve techniques like feature elimination and genetic algorithms. To accsss the usefulness of the given features, multiple feature subsets are created and corresponding models are generated. These models are individually checked for their efficiency of prediction by analyzing the error and then the best model is selected. However, these techniques are computationally very expensive due to their iterative nature. Embedded methods like decision trees are helpful in combating the problem of overfitting and are also less expensive [385,386]. Therefore, using these techniques can help to increase the efficiency of the ML model by controlling the bias and variance.

\subsection{Mixed variable problems}

Many real life problems involve datasets of mixed variables, i.e. continuous and discrete both. Discretization of data with continuous nature may result in considerable information loss. The quest of understanding the complete material behavior under specific conditions has resulted in many mixedvariable optimization problems. Specially with clustering algorithms where different distance metrics are used to draw patterns in the data based on similarity, mixed variables in the input space are challenging. To address this problem, a few algorithms like, heterogeneous Euclidean overlap metric, value difference metric and heterogeneous value difference metric are being used [387,388]. Designing the polymer composites involves consideration of both qualitative and quantitative aspects in terms of their composition and microstructure which often result in mixed variable problems [308]. For example, while analyzing laminated composite plates or shells, the number of plies is a discrete variable while other parameters like ply angle and material properties are continuous variables. Kim et 
al. [389] compared the performance of ANN and gaussian process in the presence of mixed variable input space and found that GP works better in such cases due to its interpolating nature. Parallel optimization algorithms are proven to be the most efficient ones in handling the nonconvex mixed variable problems [390]. Particle swarm optimization algorithms are also found to be effective in solving problems involving continuous and discrete variables simultaneously [391]. Adequate literature is not available on mixed variable problems concerning polymer composites despite its relevance to the highest order. Hence this area requires more attention in the near future involving optimum design under multi-objective demands.

\subsection{Robustness under the influence of noise}

Noise in the training dataset is an inevitable problem that impacts the prediction accuracy of the ML algorithms, eventually affecting the robustness of these methods. Robustness is a measure of the quality of ML algorithms which defines the performance of different methods [392]. Any algorithm is said to be robust if it has the capability of being insensitive to the inherent noise in the data [375, 376]. Bayesian decision rules and relative loss of accuracy are two conventional metrics used for measuring the degree of robustness of an algorithm [395,396]. Saez et al. [394] proposed a new evaluation measure of robustness by combining the concept of performance and robustness, referred to as equalized loss of accuracy. Every real-world problem is associated with some noise due to measurement error, equipment error, calibration error, wrong assumptions, wrongly assigned classes/labels, modeling error and many other unavoidable errors [397]. In the case of polymer composites, data obtained from tomographic studies, X-ray projection, non-destructive testing, and acoustic emission is often noisy [156,398-400]. Consequently, the pattern recognition and identification of defects in the composites become difficult and result in inaccurate predictive modeling. Many researchers have carried out various studies to understand and measure the effect of noise in the training datasets. Mukhopadhyay et al. [401] introduced artificial noise to three structures viz. a simply supported beam, a spring mass damper system and a fiber reinforced polymer composite bridge deck to evaluate the performance of central composite design and D-optimal design methods in conjunction with response surface modeling for the identification of structural damage. Both the methods are found to perform satisfactorily for all the three structures upto a noise level of $1.5 \%$. Subsequently, Mukhopadhyay et al. [402,403] quantified the effect of noise on machine learning based uncertainty quantification of polymer composites. Saseendran et al. [404] analyzed the impact of noise on two ML algorithms namely, polynomial and linear regression with ridge regularization. Both the algorithms suffered almost equal degradation in the performance accuracy with increasing percentage 
of noise in the data. Presence of noise in the dataset not only reduces the prediction and classification accuracy of a machine learning model, but also increases the learning time [405]. In order to identify noise in the data, ensemble, single learning and distance based techniques have been reported in the literature [406-408]. Ensemble based techniques have proven to be very effective in reducing the impact of noise on the damage identification of structures [409,410]. Since noise in a dataset is inevitable and the performance of ML algorithms depends significantly on quality of the dataset, it is important for the algorithm to be robust and stable enough even after some noise addition.

\subsection{Latest trends and future road maps}

The ever-growing capabilities of machine learning regularly attract the interest of researchers to exploit it to the maximum extent for solving the complexities of material science. The availability of a variety of large and complex data calls for affordable and more powerful computational processes. In this view, hybrid machine learning is a recent development with the promise of solving high dimensional problems with adequate intricacies. Hybrid machine learning works on the idea of combining multiple ML algorithms to increase the overall prediction capability by tuning mutually and generalizing or adapting to unseen data [411]. Ensemble based methods are an example of hybrid machine learning, which has been adopted by many in the areas of anomaly detection, speech recognition, uncertainty quantification and prediction of mechanical response concerning different types of composites [412-415]. A few successful attempts are reported in the area of polymer composites also. Mukhopadhyay et al. [416] reviewed the possibility of using hybrid machine learning by combining two ML algorithms for addressing the stochastic impact mechanics of polymer composites and obtained an enhanced level of computational efficiency as well as accuracy. In the hybrid ML algorithm, they showed that PCE can be used for global approximation, while the local fluctuations could be captured by kriging. Vu et al. [417] proposed a hybrid machine learning model to estimate the shear capacity of concrete strengthened with fiber-reinforced polymer composites. The hybrid ML model was based on the algorithms of support vector machine, least square and firefly algorithms. Results of this hybrid model were compared with the results obtained using artificial neural networks and the hybrid ML model showed 15\% more accuracy. Hybrid machine learning has the potential to outperform individual ML methods in general [416].

Other significant recent advances in machine learning include adaptive learning. Traditional machine learning makes use of training and prediction as two main pipelines of every algorithm but adaptive learning uses a single pipeline and works on the basis of reinforcement learning. It observes and learns from the changes in the input and the output values along with considering their allied 


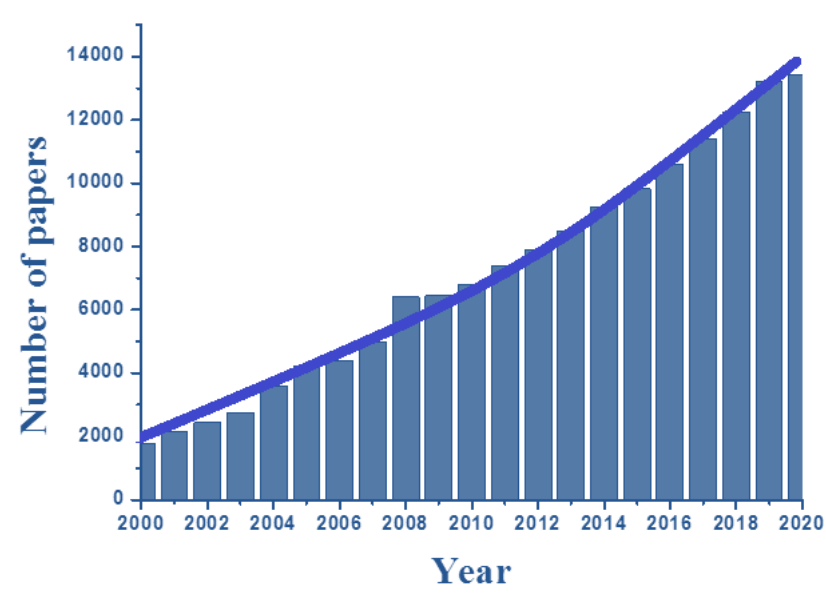

(a)

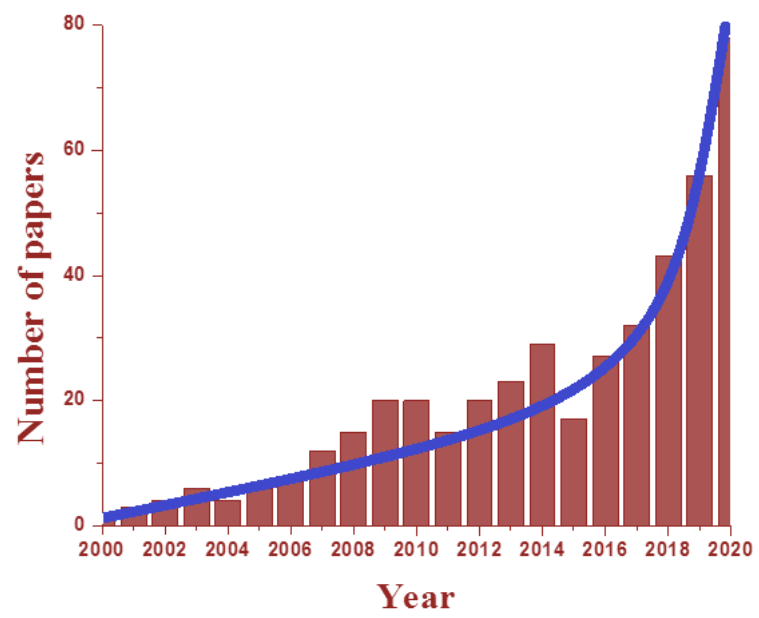

(c)

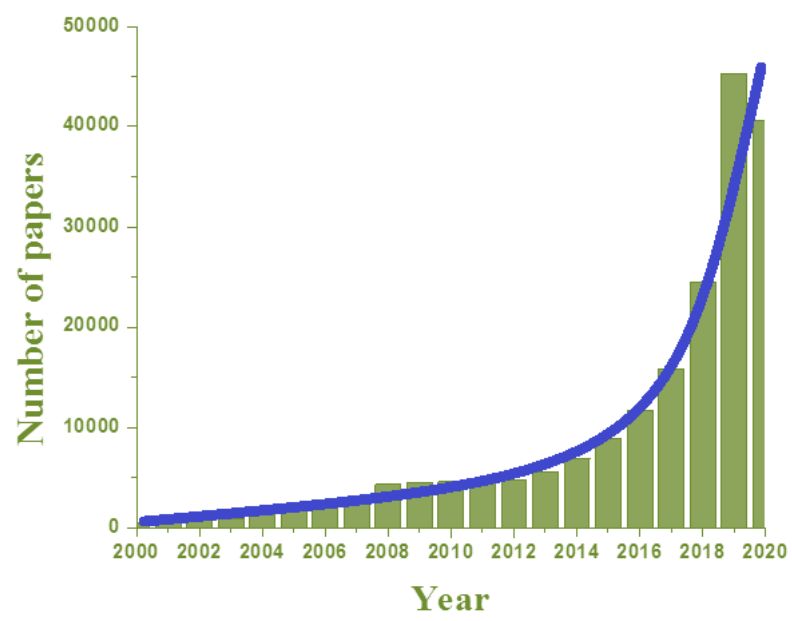

(b)

\section{Legends (Publication statistics)}

Polymer composites

Machine learning

Machine learning in polymer composites

Trend of growth

Figure 13: Publication statistics of the last two decades. (a) Number of published papers on polymer composites (b) Number of published papers on machine learning (c) Number of published papers on machine learning in polymer composites (from Scopus database). Note that the data for the year 2020 is still incomplete.

characteristics. Adaptive machine learning accepts the feedback from the working environment and then works accordingly to make improved predictions. The framework of machine learning has been found to be very promising for solving highly non-linear, dynamic systems even in the presence of uncertainties [76,418].

Multi-scale problems are very common in polymer composites as these composites are made of different phases (for details, refer to section 1). Therefore, a multi-scale analysis method is generally used to account for the size effect of the phases or the reinforcement added on the overall behavior of polymer composites. The approach of molecular dynamics has been used for nano to continuum scale bridging for developing efficient nano-composites $[419,420]$. But the molecular dynamics is considered 
computationally expensive and intractable [421]. Adaptive ML has been successfully used to build efficient scale-bridging models [422,423]. Recently seamless ML based algorithms have been presented for multi-scale optimization and uncertainty quantification of fiber-reinforced polymer composites [135]. It has been shown that a more elementary level analysis in polymer composites could lead to better insights and design of the global properties. An evolving research area with exceptional promise is the multi-fidelity modeling in machine learning. In this approach, the inexpensive low-fidelity input data and expensive high-fidelity input data can be optimally utilized for achieving a relatively high level of accuracy in efficient machine learning model formation [424, 425]. Multi-scale systems like composites can be immensely benefitted from the multi-fidelity modeling approach in the computational investigations involving machine learning.

Recently the capability of feature selection in ML algorithms has been exploited to identify the hitherto undiscovered features of composites that could affect the global responses [354]. The ability of identifying sublime system features that are not obvious from our general physical understanding is particularly useful for complex material and microstructural systems like polymer composites. Even though the major application of ML in the field of polymer composites is still limited to the efficient prediction for carrying out a plethora of computationally intensive analyses, different aspects mentioned in this section (such as hybrid ML algorithms, adaptive and reinforcement learning, MLassisted scale-bridging, mixed variable problems, multi-fidelity modeling, feature identification etc.) would lead the research of this field in the foreseeable future. Research in the area of polymer composites is increasing exponentially due to the innumerable advantages that these composites possess, wherein the implementation of machine learning is still at a nascent stage, but with extraordinary potential and striking growth rate (refer to figure 13). The comprehensive review presented in this article will help the concerned researchers and practitioners to prioritize the field of required research in exploiting the fast-evolving capabilities of machine learning for better understanding and development of multi-functional polymer composites.

\section{Conclusions}

This paper summarizes the recent progress in the applications of various machine learning algorithms concerning polymer based composites. A comprehensive account of different kinds of analyses that can be performed using ML (such as computationally efficient prediction, optimization, feature identification, uncertainty quantification, reliability and sensitivity analysis) is presented along with a general overview of commonly used algorithms and their implementation to predict the mechanical and multi-physical behavior of polymer composites. It is emphasized how the exploitation 
of ML could lead to achieving various tunable multifunctional properties in polymer composites simultaneously based on the constituent components and their arrangements or proportions. Different sampling techniques and the accuracy checking criteria for evaluating the performance of ML models are explained in detail. Challenges like the curse of dimensionality, overfitting, inevitable influence of noise, multiple-scale bridging and mixed variable problems are discussed including the current status of concerning literature in polymer composites. The latest advancements in the field of machine learning (such as hybrid algorithms, adaptive and reinforced learning, multi-fidelity modeling) which have the potential to be integrated with polymer composites in the near future are duly highlighted. Based on extensive literature survey, a few recommendations on the usage of various machine learning algorithms for addressing different critical problems concerning polymer composites are also provided along with a note of the problem-specific nature of such approaches. The current paper with a comprehensive review of literature and practical perspectives on various critical aspects of machine learning concerning polymer composites would contribute to myriad possibilities for the researchers to explore the trends, patterns, and efficient computational relations for improved characterization of existing materials and discovering new multi-functional materials.

\section{Acknowledgments}

AS and VK acknowledge the financial support received from Ministry of Education (MoE), India and the seed grant received by IIT Jammu, respectively. TM would like to acknowledge the initiation grant received from IIT Kanpur during the period of this research.

\section{Conflict of interest statement}

The authors declare that there is no conflict of interest.

\section{References}

[1] V. Kushvaha, A. Branch, H. Tippur, Effect of Loading Rate on Dynamic Fracture Behavior of Glass and Carbon Fiber Modified Epoxy, in: B. Song, D. Casem, J. Kimberley (Eds.), Dynamic Behavior of Materials, Volume 1, Springer International Publishing, 2014: pp. 169-176.

[2] M.R. Sanjay, G.R. Arpitha, L.L. Naik, K. Gopalakrishna, B. Yogesha, Applications of Natural Fibers and Its Composites: An Overview, Natural Resources. 7 (2016) 108-114. https://doi.org/10.4236/nr.2016.73011.

[3] D.A. van den Ende, H.J. van de Wiel, W.A. Groen, S. van der Zwaag, Direct strain energy harvesting in automobile tires using piezoelectric PZT-polymer composites, Smart Mater. Struct. 21 (2011) 015011. https://doi.org/10.1088/0964-1726/21/1/015011.

[4] G. Marsh, Composites get in deep with new-generation engine, Reinforced Plastics. 50 (2006) 26-29. https://doi.org/10.1016/S0034-3617(06)71188-2. 
[5] Z. Spitalsky, D. Tasis, K. Papagelis, C. Galiotis, Carbon nanotube-polymer composites: Chemistry, processing, mechanical and electrical properties, Progress in Polymer Science. 35 (2010) 357-401. https://doi.org/10.1016/j.progpolymsci.2009.09.003.

[6] Mechanical and Physical Testing of Biocomposites, Fibre-Reinforced Composites and Hybrid Composites, Elsevier, 2019. https://doi.org/10.1016/C2016-0-04437-6.

[7] E.J. Barbero, Introduction to Composite Materials Design, CRC Press, 2017.

[8] Q.H. Qin, 1 - Introduction to the composite and its toughening mechanisms, in: Q. Qin, J. Ye (Eds.), Toughening Mechanisms in Composite Materials, Woodhead Publishing, 2015: pp. 1-32. https://doi.org/10.1016/B978-1-78242-279-2.00001-9.

[9] D.K. Rajak, D.D. Pagar, R. Kumar, C.I. Pruncu, Recent progress of reinforcement materials: a comprehensive overview of composite materials, Journal of Materials Research and Technology. 8 (2019) 6354-6374. https://doi.org/10.1016/j.jmrt.2019.09.068.

[10] O. Faruk, A.K. Bledzki, H.-P. Fink, M. Sain, Biocomposites reinforced with natural fibers: 2000-2010, Progress in Polymer Science. 37 (2012) 1552-1596. https://doi.org/10.1016/j.progpolymsci.2012.04.003.

[11] M.J. John, S. Thomas, Biofibres and biocomposites, Carbohydrate Polymers. 71 (2008) 343364. https://doi.org/10.1016/j.carbpol.2007.05.040.

[12] V. Kushvaha, H. Tippur, Effect of filler shape, volume fraction and loading rate on dynamic fracture behavior of glass-filled epoxy, Composites Part B: Engineering. 64 (2014) 126-137. https://doi.org/10.1016/j.compositesb.2014.04.016.

[13] A. Esnaola, I. Tena, I. Saenz-Dominguez, J. Aurrekoetxea, I. Gallego, I. Ulacia, Effect of the manufacturing process on the energy absorption capability of GFRP crush structures, Composite Structures. 187 (2018) 316-324. https://doi.org/10.1016/j.compstruct.2017.12.079.

[14] A. Afrouzian, H. Movahhedi Aleni, G. Liaghat, H. Ahmadi, Effect of nano-particles on the tensile, flexural and perforation properties of the glass/epoxy composites, Journal of Reinforced Plastics and Composites. 36 (2017) 900-916. https://doi.org/10.1177/0731684417694753.

[15] J. Kathi, K.-Y. Rhee, J.H. Lee, Effect of chemical functionalization of multi-walled carbon nanotubes with 3-aminopropyltriethoxysilane on mechanical and morphological properties of epoxy nanocomposites, Composites Part A: Applied Science and Manufacturing. 40 (2009) 800-809. https://doi.org/10.1016/j.compositesa.2009.04.001.

[16] V. Kushvaha, H. Tippur, Effect of Filler Particle Shape on Dynamic Fracture Behavior of Glass-Filled Epoxy, in: V. Chalivendra, B. Song, D. Casem (Eds.), Dynamic Behavior of Materials, Volume 1, Springer New York, 2013: pp. 513-522.

[17] K.N. Bharath, P. Madhushri, T.G.Y. Gowda, M.R. Sanjay, V. Kushvaha, S. Siengchin, Alkaline Effect on Characterization of Discarded Waste of Moringa oleifera Fiber as a Potential Eco-friendly Reinforcement for Biocomposites, J Polym Environ. (2020). https://doi.org/10.1007/s10924-02001818-4.

[18] Z. Liu, J. Wang, V. Kushvaha, S. Poyraz, H. Tippur, S. Park, M. Kim, Y. Liu, J. Bar, H. Chen, $X$. Zhang, Poptube approach for ultrafast carbon nanotube growth, Chem. Commun. 47 (2011) 9912 9914. https://doi.org/10.1039/C1CC13359D.

[19] M.A. Osman, V. Mittal, H.R. Lusti, The Aspect Ratio and Gas Permeation in Polymer-Layered Silicate Nanocomposites, Macromol. Rapid Commun. 25 (2004) 1145-1149. https://doi.org/10.1002/marc.200400112. 
[20] V. Kushvaha, Synthesis, Processing and Dynamic Fracture Behavior of Particulate Epoxy Composites with Conventional and Hierarchical Micro-/Nano-fillers, (2016). https://etd.auburn.edu/handle/10415/5468 (accessed August 26, 2019).

[21] K.L. Reifsnider, Modelling of the interphase in polymer-matrix composite material systems, Composites. 25 (1994) 461-469. https://doi.org/10.1016/0010-4361(94)90170-8.

[22] D.A. Jesson, J.F. Watts, The Interface and Interphase in Polymer Matrix Composites: Effect on Mechanical Properties and Methods for Identification, Polymer Reviews. 52 (2012) 321-354. https://doi.org/10.1080/15583724.2012.710288.

[23] L.H. Sharpe, The Interphase in Adhesion, The Journal of Adhesion. 4 (1972) 51-64. https://doi.org/10.1080/00218467208072210.

[24] M.Y. Boluk, H.P. Schreiber, Interfacial interactions and the properties of filled polymers: I. Dynamic-mechanical responses, Polymer Composites. 7 (1986) 295-301. https://doi.org/10.1002/pc.750070506.

[25] G. Banhegyi, Interfacial Polarization and its Diagnostic Significance in Polymeric Composites, in: G. Akovali (Ed.), The Interfacial Interactions in Polymeric Composites, Springer Netherlands, Dordrecht, 1993: pp. 421-430. https://doi.org/10.1007/978-94-011-1642-8_21.

[26] H.P. Schreiber, Aspects of Component Interactions in Polymer Systems, in: G. Akovali (Ed.), The Interfacial Interactions in Polymeric Composites, Springer Netherlands, Dordrecht, 1993: pp. 2159. https://doi.org/10.1007/978-94-011-1642-8_2.

[27] J.-K. Kim, A. Hodzic, Nanoscale characterisation of thickness and properties of interphase in polymer matrix composites, The Journal of Adhesion. 79 (2003) 383-414. https://doi.org/10.1080/00218460309585.

[28] M. Rokbi, H. Osmani, A. Imad, N. Benseddiq, Effect of Chemical treatment on Flexure Properties of Natural Fiber-reinforced Polyester Composite, Procedia Engineering. 10 (2011) 20922097. https://doi.org/10.1016/j.proeng.2011.04.346.

[29] K. Joseph, S. Thomas, C. Pavithran, Effect of chemical treatment on the tensile properties of short sisal fibre-reinforced polyethylene composites, Polymer. 37 (1996) 5139-5149. https://doi.org/10.1016/0032-3861(96)00144-9.

[30] H. Deng, L. Lin, M. Ji, S. Zhang, M. Yang, Q. Fu, Progress on the morphological control of conductive network in conductive polymer composites and the use as electroactive multifunctional materials, Progress in Polymer Science. 39 (2014) 627-655. https://doi.org/10.1016/j.progpolymsci.2013.07.007.

[31] M.H. Al-Saleh, U. Sundararaj, Review of the mechanical properties of carbon nanofiber/polymer composites, Composites Part A: Applied Science and Manufacturing. 42 (2011) 2126-2142. https://doi.org/10.1016/j.compositesa.2011.08.005.

[32] J. Gassan, A study of fibre and interface parameters affecting the fatigue behaviour of natural fibre composites, Composites Part A: Applied Science and Manufacturing. 33 (2002) 369-374. https://doi.org/10.1016/S1359-835X(01)00116-6.

[33] A review on the role of interface in mechanical, thermal, and electrical properties of polymer composites | SpringerLink, (n.d.). https://link.springer.com/article/10.1007/s42114-018-0022-9 (accessed June 2, 2020).

[34] N. Patel, L.J. Lee, Effects of fiber mat architecture on void formation and removal in liquid composite molding, Polymer Composites. 16 (1995) 386-399. https://doi.org/10.1002/pc.750160507.

[35] Critical factors on manufacturing processes of natural fibre composites - ScienceDirect, (n.d.). https://www.sciencedirect.com/science/article/abs/pii/S1359836811004483 (accessed June 2, 2020). 
[36] B.S. Yoon, J.Y. Joang, M.H. Suh, Y.M. Lee, S.H. Lee, Mechanical properties of polypropylene/polyamide 6 blends: Effect of manufacturing processes and compatibilization, Polymer Composites. 18 (1997) 757-764. https://doi.org/10.1002/pc.10328.

[37] W.R. Tyson, G.J. Davies, A photoelastic study of the shear stresses associated with the transfer of stress during fibre reinforcement, Br. J. Appl. Phys. 16 (1965) 199-205. https://doi.org/10.1088/0508-3443/16/2/313.

[38] G. Meijer, F. Ellyin, Z. Xia, Aspects of residual thermal stress/strain in particle reinforced metal matrix composites, Composites Part B: Engineering. 31 (2000) 29-37. https://doi.org/10.1016/S13598368(99)00060-8.

[39] Z. Liu, L. Zhang, S. Poyraz, J. Smith, V. Kushvaha, H. Tippur, X. Zhang, An ultrafast microwave approach towards multi-component and multi-dimensional nanomaterials, RSC Adv. 4 (2014) 9308-9313. https://doi.org/10.1039/C3RA47086E.

[40] M. Hemath, S.M. Rangappa, V. Kushvaha, H.N. Dhakal, S. Siengchin, A comprehensive review on mechanical, electromagnetic radiation shielding, and thermal conductivity of fibers/inorganic fillers reinforced hybrid polymer composites, Polymer Composites. n/a (n.d.). https://doi.org/10.1002/pc.25703.

[41] A. Haque, A. Ramasetty, Theoretical study of stress transfer in carbon nanotube reinforced polymer matrix composites, Composite Structures. $71 \quad$ (2005) 68-77. https://doi.org/10.1016/j.compstruct.2004.09.029.

[42] L.N. McCartney, A. Kelly, New theoretical model of stress transfer between fibre and matrix in a uniaxially fibre-reinforced composite, Proceedings of the Royal Society of London. A. Mathematical and Physical Sciences. 425 (1989) 215-244. https://doi.org/10.1098/rspa.1989.0104.

[43] V. Kushvaha, S. Anandkumar, P. Madhushri, Dynamic fracture toughness index: A new integrated methodology for mode-I fracture behaviour of polymer composite under impact loading, Mater. Res. Express. (2019). https://doi.org/10.1088/2053-1591/ab4e35.

[44] T. Rossman, V. Kushvaha, D. Dragomir-Daescu, QCT/FEA predictions of femoral stiffness are strongly affected by boundary condition modeling, Computer Methods in Biomechanics and Biomedical Engineering. 19 (2016) 208-216. https://doi.org/10.1080/10255842.2015.1006209.

[45] B.R. Isanaka, M.A. Akbar, B.P. Mishra, V. Kushvaha, Free vibration analysis of thin plates: Bare versus Stiffened, Eng. Res. Express. 2 (2020) 015014. https://doi.org/10.1088/2631-8695/ab6264.

[46] A. Garg, N.G. Reddy, H. Huang, P. Buragohain, V. Kushvaha, Modelling contaminant transport in fly ash-bentonite composite landfill liner: mechanism of different types of ions, Scientific Reports. 10 (2020) 11330. https://doi.org/10.1038/s41598-020-68198-6.

[47] B.R.I. Isanaka, A.A. M, V. Kushvaha, Static Analysis of Thin Plates: Bare and Stiffened, INCAS BULLETIN. 12 (2020) 67-81. https://doi.org/10.13111/2066-8201.2020.12.1.7.

[48] S.J.V. Frankland, V.M. Harik, G.M. Odegard, D.W. Brenner, T.S. Gates, The stress-strain behavior of polymer-nanotube composites from molecular dynamics simulation, Composites Science and Technology. 63 (2003) 1655-1661. https://doi.org/10.1016/S0266-3538(03)00059-9.

[49] Y. Li, S. Wang, Q. Wang, M. Xing, Enhancement of fracture properties of polymer composites reinforced by carbon nanotubes: A molecular dynamics study, Carbon. 129 (2018) 504-509. https://doi.org/10.1016/j.carbon.2017.12.029.

[50] A. Garg, H. Huang, V. Kushvaha, P. Madhushri, V. Kamchoom, I. Wani, N. Koshy, H.-H. Zhu, Mechanism of biochar soil pore-gas-water interaction: gas properties of biochar-amended sandy soil at different degrees of compaction using KNN modeling, Acta Geophys. 68 (2020) 207-217. https://doi.org/10.1007/s11600-019-00387-y. 
[51] I. Wani, A. Sharma, V. Kushvaha, P. Madhushri, L. Peng, Effect of pH, Volatile Content, and Pyrolysis Conditions on Surface Area and O/C and H/C Ratios of Biochar: Towards Understanding Performance of Biochar Using Simplified Approach, Journal of Hazardous, Toxic, and Radioactive Waste. 24 (2020) 04020048. https://doi.org/10.1061/(ASCE)HZ.2153-5515.0000545.

[52] G.X. Gu, C.-T. Chen, D.J. Richmond, M.J. Buehler, Bioinspired hierarchical composite design using machine learning: simulation, additive manufacturing, and experiment, Mater. Horiz. 5 (2018) 939-945. https://doi.org/10.1039/C8MH00653A.

[53] P. Pattnaik, A. Sharma, M. Choudhary, V. Singh, P. Agarwal, V. Kukshal, Role of machine learning in the field of Fiber reinforced polymer composites: A preliminary discussion, Materials Today: Proceedings. (2020). https://doi.org/10.1016/j.matpr.2020.11.026.

[54] D.O. Hebb, The organization of behavior, JOHN WILEY \& SONS, Inc., New York, 1949.

[55] Delving Deep into Rectifiers | Proceedings of the 2015 IEEE International Conference on Computer Vision (ICCV), (n.d.). https://dl.acm.org/doi/10.1109/ICCV.2015.123 (accessed June 12, 2020).

[56] Readings in Speech Recognition - 1st Edition, (n.d.). https://www.elsevier.com/books/readingsin-speech-recognition/waibel/978-0-08-051584-7 (accessed June 12, 2020).

[57] M. Bojarski, D. Del Testa, D. Dworakowski, B. Firner, B. Flepp, P. Goyal, L.D. Jackel, M. Monfort, U. Muller, J. Zhang, X. Zhang, J. Zhao, K. Zieba, End to End Learning for Self-Driving Cars, ArXiv:1604.07316 [Cs]. (2016). http://arxiv.org/abs/1604.07316 (accessed June 12, 2020).

[58] P.K. Chan, S.J. Stolfo, Toward Scalable Learning with Non-Uniform Class and Cost Distributions: A Case Study in Credit Card Fraud Detection, (n.d.) 5.

[59] J. Schmidt, M.R.G. Marques, S. Botti, M.A.L. Marques, Recent advances and applications of machine learning in solid-state materials science, Npj Comput Mater. 5 (2019) 83. https://doi.org/10.1038/s41524-019-0221-0.

[60] S. Webb, Deep learning for biology, Nature. $554 \quad$ (2018) 555-557. https://doi.org/10.1038/d41586-018-02174-z.

[61] G.B. Goh, N.O. Hodas, A. Vishnu, Deep learning for computational chemistry, J. Comput. Chem. 38 (2017) 1291-1307. https://doi.org/10.1002/jcc.24764.

[62] C. Xu, S.A. Jackson, Machine learning and complex biological data, Genome Biology. 20 (2019) 76. https://doi.org/10.1186/s13059-019-1689-0.

[63] Y. Liu, C. Niu, Z. Wang, Y. Gan, Y. Zhu, S. Sun, T. Shen, Machine learning in materials genome initiative: A review, Journal of Materials Science \& Technology. 57 (2020) 113-122. https://doi.org/10.1016/j.jmst.2020.01.067.

[64] G.B. Olson, Designing a New Material World, Science. 288 (2000) 993-998. https://doi.org/10.1126/science.288.5468.993.

[65] Y.M.F. El Hasadi, J.T. Padding, Solving fluid flow problems using semi-supervised symbolic regression on sparse data, AIP Advances. 9 (2019) 115218. https://doi.org/10.1063/1.5116183.

[66] M. Riedmiller, Advanced supervised learning in multi-layer perceptrons — From backpropagation to adaptive learning algorithms, Computer Standards \& Interfaces. 16 (1994) 265278. https://doi.org/10.1016/0920-5489(94)90017-5.

[67] M.-L. Zhang, Z.-H. Zhou, A Review on Multi-Label Learning Algorithms, IEEE Transactions on Knowledge and Data Engineering. 26 (2014) 1819-1837. https://doi.org/10.1109/TKDE.2013.39.

[68] H.B. Barlow, Unsupervised Learning, Neural Computation. 1 (1989) 295-311. https://doi.org/10.1162/neco.1989.1.3.295. 
[69] M.A.T. Figueiredo, A.K. Jain, Unsupervised learning of finite mixture models, IEEE Transactions on Pattern Analysis and Machine Intelligence. 24 (2002) 381-396. https://doi.org/10.1109/34.990138.

[70] D.J. Hand, Principles of Data Mining, Drug-Safety. 30 (2007) 621-622. https://doi.org/10.2165/00002018-200730070-00010.

[71] Sampling and Splitting Data, (n.d.). https://developers.google.com/machine-learning/dataprep/construct/sampling-splitting/sampling (accessed June 20, 2020).

[72] Y. Xu, R. Goodacre, On Splitting Training and Validation Set: A Comparative Study of CrossValidation, Bootstrap and Systematic Sampling for Estimating the Generalization Performance of Supervised Learning, J. Anal. Test. 2 (2018) 249-262. https://doi.org/10.1007/s41664-018-0068-2.

[73] P. Langley, Selection of Relevant Features in Machine Learning.:. Defense Technical Information Center, Fort Belvoir, VA, 1994. https://doi.org/10.21236/ADA292575.

[74] L. Yu, H. Liu, Efficient Feature Selection via Analysis of Relevance and Redundancy, Journal of Machine Learning Research. 5 (2004) 1205-1224.

[75] L.M. Ghiringhelli, J. Vybiral, S.V. Levchenko, C. Draxl, M. Scheffler, Big Data of Materials Science: Critical Role of the Descriptor, Phys. Rev. Lett. 114 (2015) 105503. https://doi.org/10.1103/PhysRevLett.114.105503.

[76] V. Botu, R. Ramprasad, Adaptive machine learning framework to accelerate ab initio molecular dynamics, Undefined. (2015). /paper/Adaptive-machine-learning-framework-to-accelerate-BotuRamprasad/c9934d684fcc0b8ac6ed25b34d96e726cf2d7b99 (accessed June 21, 2020).

[77] D. Sleeman, M. Rissakis, S. Craw, N. Graner, S. Sharma, Consultant-2: pre- and postprocessing of Machine Learning applications, International Journal of Human-Computer Studies. 43 (1995) 43-63. https://doi.org/10.1006/ijhc.1995.1035.

[78] S. Sharma, J. Agrawal, S. Sharma, Classification Through Machine Learning Technique: C4. 5 Algorithm based on Various Entropies, IJCA. 82 (2013) 28-32. https://doi.org/10.5120/14249-2444.

[79] P.M. Pardalos, G. Xue, Algorithms for a Class of Isotonic Regression Problems, Algorithmica. 23 (1999) 211-222. https://doi.org/10.1007/PL00009258.

[80] G. Pilania, C. Wang, X. Jiang, S. Rajasekaran, R. Ramprasad, Accelerating materials property predictions using machine learning, Scientific Reports. 3 (2013) 2810. https://doi.org/10.1038/srep02810.

[81] V. Daghigh, T.E. Lacy, H. Daghigh, G. Gu, K.T. Baghaei, M.F. Horstemeyer, C.U. Pittman, Heat deflection temperatures of bio-nano-composites using experiments and machine learning $\begin{array}{llllll}\text { predictions, } & \text { Materials } & \text { Today } & \text { Communications. } & 22 & \text { (2020) }\end{array}$ https://doi.org/10.1016/j.mtcomm.2019.100789.

[82] Z. Qi, N. Zhang, Y. Liu, W. Chen, Prediction of mechanical properties of carbon fiber based on cross-scale FEM and machine learning, Composite Structures. 212 (2019) 199-206. https://doi.org/10.1016/j.compstruct.2019.01.042.

[83] M. Francisco, S. Revollar, P. Vega, R. Lamanna, A comparative study of deterministic and stochastic optimization methods for integrated design of processes, IFAC Proceedings Volumes. 38 (2005) 335-340. https://doi.org/10.3182/20050703-6-CZ-1902.00917.

[84] S. Sun, A review of deterministic approximate inference techniques for Bayesian machine learning, Neural Comput \& Applic. 23 (2013) 2039-2050. https://doi.org/10.1007/s00521-013-1445-4.

[85] H.T.C. Pedro, C.F.M. Coimbra, M. David, P. Lauret, Assessment of machine learning techniques for deterministic and probabilistic intra-hour solar forecasts, Renewable Energy. 123 (2018) 191-203. https://doi.org/10.1016/j.renene.2018.02.006. 
[86] T. Chatterjee, S. Chakraborty, R. Chowdhury, A Critical Review of Surrogate Assisted Robust Design Optimization, Arch Computat Methods Eng. 26 (2019) 245-274. https://doi.org/10.1007/s11831-017-9240-5.

[87] B. RAMAKRISHNAN, S.S. RAO, A General Loss Function Based Optimization Procedure for Robust Design, Engineering Optimization. 25 (1996) 255-276. https://doi.org/10.1080/03052159608941266.

[88] S. Sarkar, S. Vinay, R. Raj, J. Maiti, P. Mitra, Application of optimized machine learning techniques for prediction of occupational accidents, Computers \& Operations Research. 106 (2019) 210-224. https://doi.org/10.1016/j.cor.2018.02.021.

[89] K. Kalita, T. Mukhopadhyay, P. Dey, S. Haldar, Genetic programming-assisted multi-scale optimization for multi-objective dynamic performance of laminated composites: the advantage of more elementary-level analyses, Neural Comput \& Applic. 32 (2020) 7969-7993. https://doi.org/10.1007/s00521-019-04280-z.

[90] L. Sidi Salah, M. Chouai, Y. Danlée, I. Huynen, N. Ouslimani, Simulation and Optimization of Electromagnetic Absorption of Polycarbonate/CNT Composites Using Machine Learning, Micromachines. 11 (2020) 778. https://doi.org/10.3390/mi11080778.

[91] Optimization of Process Parameters for Powder Bed Fusion Additive Manufacturing by Combination of Machine Learning and Finite Element Method: A Conceptual Framework, Procedia CIRP. 67 (2018) 227-232. https://doi.org/10.1016/j.procir.2017.12.204.

[92] M. Arian Nik, K. Fayazbakhsh, D. Pasini, L. Lessard, Surrogate-based multi-objective optimization of a composite laminate with curvilinear fibers, Composite Structures. 94 (2012) 23062313. https://doi.org/10.1016/j.compstruct.2012.03.021.

[93] K. Duan, Y. He, Y. Li, J. Liu, J. Zhang, Y. Hu, R. Lin, X. Wang, W. Deng, L. Li, Machinelearning assisted coarse-grained model for epoxies over wide ranges of temperatures and cross-linking degrees, Materials \& Design. 183 (2019) 108130. https://doi.org/10.1016/j.matdes.2019.108130.

[94] L. Marín, D. Trias, P. Badalló, G. Rus, J.A. Mayugo, Optimization of composite stiffened panels under mechanical and hygrothermal loads using neural networks and genetic algorithms, Composite Structures. 94 (2012) 3321-3326. https://doi.org/10.1016/j.compstruct.2012.04.024.

[95] R. Sreenivasulu, Optimization of Surface Roughness and Delamination Damage of GFRP Composite Material in End Milling Using Taguchi Design Method and Artificial Neural Network, Procedia Engineering. 64 (2013) 785-794. https://doi.org/10.1016/j.proeng.2013.09.154.

[96] Evaluation of particle swarm optimization for strength determination of tropical wood polymer composite ProQuest, (n.d.). https://search.proquest.com/openview/511fbf008b6d2a46baf11b7bde137245/1?pqorigsite $=$ gscholar $\& c b l=1686339($ accessed October 21,2020$)$.

[97] B.O.P. Soepangkat, B. Pramujati, M.K. Effendi, R. Norcahyo, A.M. Mufarrih, Multi-objective Optimization in Drilling Kevlar Fiber Reinforced Polymer Using Grey Fuzzy Analysis and Backpropagation Neural Network-Genetic Algorithm (BPNN-GA) Approaches, Int. J. Precis. Eng. Manuf. 20 (2019) 593-607. https://doi.org/10.1007/s12541-019-00017-z.

[98] Y.D. Boon, S.C. Joshi, S.K. Bhudolia, G. Gohel, Recent Advances on the Design Automation for Performance-Optimized Fiber Reinforced Polymer Composite Components, Journal of Composites Science. 4 (2020) 61. https://doi.org/10.3390/jcs4020061.

[99] K.M. Hamdia, X. Zhuang, T. Rabczuk, An efficient optimization approach for designing machine learning models based on genetic algorithm, Neural Comput \& Applic. (2020). https://doi.org/10.1007/s00521-020-05035-X. 
[100] C. Li, D. Rubín de Celis Leal, S. Rana, S. Gupta, A. Sutti, S. Greenhill, T. Slezak, M. Height, S. Venkatesh, Rapid Bayesian optimisation for synthesis of short polymer fiber materials, Scientific Reports. 7 (2017) 5683. https://doi.org/10.1038/s41598-017-05723-0.

[101] H. Xu, R. Liu, A. Choudhary, W. Chen, A Machine Learning-Based Design Representation Method for Designing Heterogeneous Microstructures, J. Mech. Des. 137 (2015). https://doi.org/10.1115/1.4029768.

[102] R.R. Kumar, T. Mukhopadhyay, S. Naskar, K.M. Pandey, S. Dey, Stochastic low-velocity impact analysis of sandwich plates including the effects of obliqueness and twist, Thin-Walled Structures. 145 (2019) 106411. https://doi.org/10.1016/j.tws.2019.106411.

[103] S. Naskar, T. Mukhopadhyay, S. Sriramula, Spatially varying fuzzy multi-scale uncertainty propagation in unidirectional fibre reinforced composites, Composite Structures. 209 (2019) 940-967. https://doi.org/10.1016/j.compstruct.2018.09.090.

[104] S. Dey, T. Mukhopadhyay, S. Naskar, T. Dey, H. Chalak, S. Adhikari, Probabilistic characterisation for dynamics and stability of laminated soft core sandwich plates, Jnl of Sandwich Structures \& Materials. 21 (2019) 366-397. https://doi.org/10.1177/1099636217694229.

[105] P.K. Karsh, T. Mukhopadhyay, S. Dey, Stochastic low-velocity impact on functionally graded plates: Probabilistic and non-probabilistic uncertainty quantification, Composites Part B: Engineering. 159 (2019) 461-480. https://doi.org/10.1016/j.compositesb.2018.09.066.

[106] T. Mukhopadhyay, S. Chakraborty, S. Dey, S. Adhikari, R. Chowdhury, A Critical Assessment of Kriging Model Variants for High-Fidelity Uncertainty Quantification in Dynamics of composite Shells, Arch Computat Methods Eng. 24 (2017) 495-518. https://doi.org/10.1007/s11831-016-9178-z.

[107] G. Lin, Uncertainty Quantification and Scientific Machine Learning for Complex Engineering Systems, (2020). https://nanohub.org/resources/32565.

[108] S. Shekhar, K.B. Ozutemiz, R. Onler, S. Nahata, O.B. Ozdoganlar, Uncertainty quantification for polymer micromilling force models using Bayesian inference, Procedia Manufacturing. 48 (2020) 611-618. https://doi.org/10.1016/j.promfg.2020.05.089.

[109] B. Hammer, T. Villmann, How to process uncertainty in machine learning?, (2007) 12.

[110] S. Naskar, T. Mukhopadhyay, S. Sriramula, S. Adhikari, Stochastic natural frequency analysis of damaged thin-walled laminated composite beams with uncertainty in micromechanical properties, Composite Structures. 160 (2017) 312-334. https://doi.org/10.1016/j.compstruct.2016.10.035.

[111] Uncertainty Quantification in Laminated Composites: A Meta-model Based Approach, Routledge \& CRC Press. (n.d.). https://www.routledge.com/Uncertainty-Quantification-in-LaminatedComposites-A-Meta-model-Based-Approach/Dey-Mukhopadhyay-Adhikari/p/book/9781498784450 (accessed October 30, 2020).

[112] R. Bostanabad, B. Liang, J. Gao, W.K. Liu, J. Cao, D. Zeng, X. Su, H. Xu, Y. Li, W. Chen, Uncertainty quantification in multiscale simulation of woven fiber composites, Computer Methods in Applied Mechanics and Engineering. 338 (2018) 506-532. https://doi.org/10.1016/j.cma.2018.04.024.

[113] J. Doh, S.-I. Park, Q. Yang, N. Raghavan, Uncertainty quantification of percolating electrical conductance for wavy carbon nanotube-filled polymer nanocomposites using Bayesian inference, Carbon. 172 (2021) 308-323. https://doi.org/10.1016/j.carbon.2020.09.092.

[114] A. Jha, A. Chandrasekaran, C. Kim, R. Ramprasad, Impact of dataset uncertainties on machine learning model predictions: the example of polymer glass transition temperatures, Modelling Simul. Mater. Sci. Eng. 27 (2019) 024002. https://doi.org/10.1088/1361-651X/aaf8ca.

[115] X. Peng, D. Li, H. Wu, Z. Liu, J. Li, S. Jiang, J. Tan, Uncertainty analysis of composite laminated plate with data-driven polynomial chaos expansion method under insufficient input data of 
uncertain parameters, $\quad$ Composite $\quad$ Structures. $\quad 209 \quad$ (2019) 625-633. https://doi.org/10.1016/j.compstruct.2018.11.015.

[116] Z.G. Ghauch, V. Aitharaju, W.R. Rodgers, P. Pasupuleti, A. Dereims, R.G. Ghanem, Integrated stochastic analysis of fiber composites manufacturing using adapted polynomial chaos expansions, Composites Part A: Applied Science and Manufacturing. 118 (2019) 179-193. https://doi.org/10.1016/j.compositesa.2018.12.029.

[117] C. Schwab, J. Zech, Deep learning in high dimension: Neural network expression rates for generalized polynomial chaos expansions in UQ, Anal. Appl. 17 (2018) 19-55. https://doi.org/10.1142/S0219530518500203.

[118] M. Thapa, S.B. Mulani, R.W. Walters, Stochastic multi-scale modeling of carbon fiber reinforced composites with polynomial chaos, Composite Structures. 213 (2019) 82-97. https://doi.org/10.1016/j.compstruct.2019.01.068.

[119] S. Dey, T. Mukhopadhyay, H.H. Khodaparast, S. Adhikari, Fuzzy uncertainty propagation in composites using Gram-Schmidt polynomial chaos expansion, Applied Mathematical Modelling. 40 (2016) 4412-4428. https://doi.org/10.1016/j.apm.2015.11.038.

[120] K. Naresh, K. Shankar, R. Velmurugan, Reliability analysis of tensile strengths using Weibull distribution in glass/epoxy and carbon/epoxy composites, Composites Part B: Engineering. 133 (2018) 129-144. https://doi.org/10.1016/j.compositesb.2017.09.002.

[121] X. He, H. Xu, H. Sabetamal, D. Sheng, Machine learning aided stochastic reliability analysis of spatially variable slopes, Computers and Geotechnics. $126 \quad$ (2020) 103711. https://doi.org/10.1016/j.compgeo.2020.103711.

[122] L.H. Shuang, L.Z. Zhou, Y. zhu Feng, Support vector machines for structural reliability analysis, Applied Mathematics and Mechanics. (n.d.). https://doi.org/(DOI): 10.1007/s 10483-006.

[123] A. Behnia, N. Ranjbar, H.K. Chai, M. Masaeli, Failure prediction and reliability analysis of ferrocement composite structures by incorporating machine learning into acoustic emission monitoring technique, Construction and Building Materials. 122 (2016) 823-832. https://doi.org/10.1016/j.conbuildmat.2016.06.130.

[124] E.F. Alsina, M. Chica, K. Trawiński, R. Alberto, On the use of machine learning methods to predict component reliability from data-driven industrial case studies, The International Journal of Advanced Manufacturing Technology. 94 (2018) 2419-2433. https://doi.org/10.1007/s00170-0171039-x.

[125] F. Yan, Z. Lin, New strategy for anchorage reliability assessment of GFRP bars to concrete using hybrid artificial neural network with genetic algorithm, Composites Part B: Engineering. 92 (2016) 420-433. https://doi.org/10.1016/j.compositesb.2016.02.008.

[126] X. Tan, W. Bi, X. Hou, W. Wang, Reliability analysis using radial basis function networks and support vector machines, Computers and Geotechnics. 38 (2011) 178-186. https://doi.org/10.1016/j.compgeo.2010.11.002.

[127] A. Ahmad, Q. uz Z. Khan, A. Raza, Reliability analysis of strength models for CFRP-confined $\begin{array}{lllll}\text { concrete } \quad \text { cylinders, } & \text { Composite } & \text { Structures. } & 244 & \text { (2020) }\end{array}$ https://doi.org/10.1016/j.compstruct.2020.112312.

[128] G. Su, B. Yu, Y. Xiao, L. Yan, Gaussian Process Machine-Learning Method for Structural Reliability Analysis, Advances in Structural Engineering. 17 (2014) 1257-1270. https://doi.org/10.1260/1369-4332.17.9.1257. 
[129] M. Shahnewaz, R. Machial, M.S. Alam, A. Rteil, Optimized shear design equation for slender concrete beams reinforced with FRP bars and stirrups using Genetic Algorithm and reliability analysis, Engineering Structures. 107 (2016) 151-165. https://doi.org/10.1016/j.engstruct.2015.10.049.

[130] Z.-H. Shen, J.-J. Wang, J.-Y. Jiang, S.X. Huang, Y.-H. Lin, C.-W. Nan, L.-Q. Chen, Y. Shen, Phase-field modeling and machine learning of electric-thermal-mechanical breakdown of polymerbased dielectrics, Nature Communications. 10 (2019) 1843. https://doi.org/10.1038/s41467-019-098748.

[131] H.M. Gomes, A.M. Awruch, P.A.M. Lopes, Reliability based optimization of laminated composite structures using genetic algorithms and Artificial Neural Networks, Structural Safety. 33 (2011) 186-195. https://doi.org/10.1016/j.strusafe.2011.03.001.

[132] Y. Foo, C. Goh, Y. Li, Machine Learning with Sensitivity Analysis to Determine Key Factors Contributing to Energy Consumption in Cloud Data Centers, 2016. https://doi.org/10.1109/ICCCRI.2016.24.

[133] P.K. Karsh, T. Mukhopadhyay, S. Chakraborty, S. Naskar, S. Dey, A hybrid stochastic sensitivity analysis for low-frequency vibration and low-velocity impact of functionally graded plates,

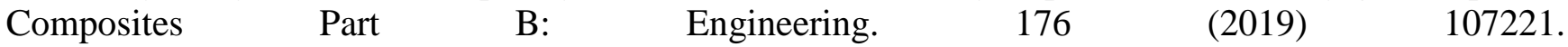
https://doi.org/10.1016/j.compositesb.2019.107221.

[134] S. Dey, T. Mukhopadhyay, S. Adhikari, Stochastic free vibration analysis of angle-ply composite plates - A RS-HDMR approach, Composite Structures. 122 (2015) 526-536. https://doi.org/10.1016/j.compstruct.2014.09.057.

[135] S. Naskar, T. Mukhopadhyay, S. Sriramula, Probabilistic micromechanical spatial variability quantification in laminated composites, Composites Part B: Engineering. 151 (2018) 291-325. https://doi.org/10.1016/j.compositesb.2018.06.002.

[136] M. Ramu, R.V. Prabhu, METAMODEL BASED ANALYSIS AND ITS APPLICATIONS: A REVIEW, (n.d.) 10.

[137] S.-H. Kim, S.-W. Na, Response surface method using vector projected sampling points, Structural Safety. 19 (1997) 3-19. https://doi.org/10.1016/S0167-4730(96)00037-9.

[138] M. Tabatabaei, J. Hakanen, M. Hartikainen, K. Miettinen, K. Sindhya, A survey on handling computationally expensive multiobjective optimization problems using surrogates: non-nature inspired methods, Struct Multidisc Optim. 52 (2015) 1-25. https://doi.org/10.1007/s00158-015-1226-z.

[139] T.W. Simpson, J.D. Poplinski, P.N. Koch, J.K. Allen, Metamodels for Computer-based Engineering Design: Survey and recommendations, EWC. 17 (2001) 129-150. https://doi.org/10.1007/PL00007198.

[140] T. Mukhopadhyay, T.K. Dey, R. Chowdhury, A. Chakrabarti, Structural Damage Identification Using Response Surface-Based Multi-objective Optimization: A Comparative Study, Arab J Sci Eng. 40 (2015) 1027-1044. https://doi.org/10.1007/s13369-015-1591-3.

[141] J.C. Helton, J.D. Johnson, C.J. Sallaberry, C.B. Storlie, Survey of sampling-based methods for uncertainty and sensitivity analysis, Reliability Engineering \& System Safety. 91 (2006) 1175-1209. https://doi.org/10.1016/j.ress.2005.11.017.

[142] K.-T. Fang, D.K.J. Lin, P. Winker, Y. Zhang, Uniform Design: Theory and Application, Technometrics. 42 (2000) 237-248. https://doi.org/10.1080/00401706.2000.10486045.

[143] S. Chen, X. Hong, C.J. Harris, Sparse kernel regression modeling using combined locally regularized orthogonal least squares and D-optimality experimental design, IEEE Transactions on Automatic Control. 48 (2003) 1029-1036. https://doi.org/10.1109/TAC.2003.812790. 
[144] An Efficient Sampling Technique for Off-line Quality Control: Technometrics: Vol 39, No 3, (n.d.). https://www.tandfonline.com/doi/abs/10.1080/00401706.1997.10485122 (accessed November 10, 2020).

[145] Orthogonal Arrays, (n.d.). http://neilsloane.com/doc/OA.html (accessed November 10, 2020).

[146] A.B. Owen, Orthogonal arrays for computer experiments, integration and visualization, Statistica Sinica. 2 (1992) 439-452.

[147] F.M. Alam, K.R. McNaught, T.J. Ringrose, A comparison of experimental designs in the development of a neural network simulation metamodel, Simulation Modelling Practice and Theory. 12 (2004) 559-578. https://doi.org/10.1016/j.simpat.2003.10.006.

[148] G. Wang, S. Shan, Review of Metamodeling Techniques for Product Design with Computationintensive Processes, Proceedings of the Canadian Engineering Education Association. (2011). https://doi.org/10.24908/pceea.v0i0.3940.

[149] P. Shah, V. Halls, J. Zheng, R. Batra, Optimal cure cycle parameters for minimizing residual stresses in fiber-reinforced polymer composite laminates, Journal of Composite Materials. 52 (2018) 773-792. https://doi.org/10.1177/0021998317714317.

[150] J. Johnston, A. Chattopadhyay, Effect of Material Variability on Multiscale Modeling of RateDependent Composite Materials, Journal of Aerospace Engineering. 28 (2015) 04015003. https://doi.org/10.1061/(ASCE)AS.1943-5525.0000488.

[151] S. Basavarajappa, K.V. Arun, J.P. Davim, Effect of Filler Materials on Dry Sliding Wear Behavior of Polymer Matrix Composites - A Taguchi Approach, Journal of Minerals and Materials Characterization and Engineering. 08 (2009) 379.

[152] R. Ghelich, M.R. Jahannama, H. Abdizadeh, F.S. Torknik, M.R. Vaezi, Central composite design (CCD)-Response surface methodology (RSM) of effective electrospinning parameters on PVPB-Hf hybrid nanofibrous composites for synthesis of HfB2-based composite nanofibers, Composites Part B: Engineering. 166 (2019) 527-541. https://doi.org/10.1016/j.compositesb.2019.01.094.

[153] Q. Li, H. Xu, F. Li, P. Li, L. Shen, J. Zhai, Synthesis of geopolymer composites from blends of CFBC fly and bottom ashes, Fuel. 97 (2012) 366-372. https://doi.org/10.1016/j.fuel.2012.02.059.

[154] S. Dey, T. Mukhopadhyay, S. Adhikari, Metamodel based high-fidelity stochastic analysis of composite laminates: A concise review with critical comparative assessment, Composite Structures. 171 (2017) 227-250. https://doi.org/10.1016/j.compstruct.2017.01.061.

[155] T. Mukhopadhyay, A multivariate adaptive regression splines based damage identification methodology for web core composite bridges including the effect of noise, Jnl of Sandwich Structures \& Materials. 20 (2018) 885-903. https://doi.org/10.1177/1099636216682533.

[156] Y. Liu, K. Liu, Z. Gao, Y. Yao, S. Sfarra, H. Zhang, X.P.V. Maldague, Non-destructive defect evaluation of polymer composites via thermographic data analysis: A manifold learning method, Infrared Physics \& Technology. 97 (2019) 300-308. https://doi.org/10.1016/j.infrared.2019.01.008.

[157] D.S. Balreira, L.A.G. Moura, E. Parente Junior, Sequential approximate optimization of composite structures using radial basis functions, in: Proceedings of the 4th Brazilian Conference on Composite Materials, Pontifícia Universidade Católica do Rio de Janeiro, 2018: pp. 939-946. https://doi.org/10.21452/bccm4.2018.16.010.

[158] R. Zare-Dorabei, S.M. Ferdowsi, A. Barzin, A. Tadjarodi, Highly efficient simultaneous ultrasonic-assisted adsorption of $\mathrm{Pb}$ (II), $\mathrm{Cd}$ (II), $\mathrm{Ni}$ (II) and $\mathrm{Cu}$ (II) ions from aqueous solutions by graphene oxide modified with 2,2'-dipyridylamine: Central composite design optimization, Ultrasonics Sonochemistry. 32 (2016) 265-276. https://doi.org/10.1016/j.ultsonch.2016.03.020. 
[159] Dave Anderson, George McNeill, Artificial neural networks technology, Rome Laboratory RL/C3C Griffiss AFB, NY 13441-5700, 1992.

[160] N. Wanas, G. Auda, M.S. Kamel, F. Karray, On the optimal number of hidden nodes in a neural network, in: Conference Proceedings. IEEE Canadian Conference on Electrical and Computer $\begin{array}{lllll}\text { Engineering (Cat. } & \text { No.98TH8341), } & \text { 1998: } & \text { pp. }\end{array}$ https://doi.org/10.1109/CCECE.1998.685648.

[161] J.C. Patra, R.N. Pal, B.N. Chatterji, G. Panda, Identification of nonlinear dynamic systems using functional link artificial neural networks, IEEE Transactions on Systems, Man, and Cybernetics, Part B (Cybernetics). 29 (1999) 254-262. https://doi.org/10.1109/3477.752797.

[162] H. Moradkhani, K. Hsu, H.V. Gupta, S. Sorooshian, Improved streamflow forecasting using self-organizing radial basis function artificial neural networks, Journal of Hydrology. 295 (2004) 246262. https://doi.org/10.1016/j.jhydrol.2004.03.027.

[163] P. Sibi, S.A. Jones, P. Siddarth, Analysis of different activation functions using back propagation neural networks, .. Vol. 47 (2005) 5.

[164] S. Dreiseitl, L. Ohno-Machado, Logistic regression and artificial neural network classification models: a methodology review, Journal of Biomedical Informatics. 35 (2002) 352-359. https://doi.org/10.1016/S1532-0464(03)00034-0.

[165] O.I. Abiodun, A. Jantan, A.E. Omolara, K.V. Dada, N.A. Mohamed, H. Arshad, State-of-the-art in artificial neural network applications: A survey, Heliyon. 4 (2018) e00938. https://doi.org/10.1016/j.heliyon.2018.e00938.

[166] M. Lynch, H. Patel, A. Abrahamse, A.R. Rajendran, L. Medsker, Neural network applications in physics, in: IJCNN'01. International Joint Conference on Neural Networks. Proceedings (Cat. No.01CH37222), 2001: pp. 2054-2058 vol.3. https://doi.org/10.1109/IJCNN.2001.938482.

[167] O. Araque, I. Corcuera-Platas, J.F. Sánchez-Rada, C.A. Iglesias, Enhancing deep learning sentiment analysis with ensemble techniques in social applications, Expert Systems with Applications. 77 (2017) 236-246. https://doi.org/10.1016/j.eswa.2017.02.002.

[168] Application of artificial intelligence techniques in the petroleum industry: a review, Springerprofessional.De. (n.d.). https://www.springerprofessional.de/en/application-of-artificialintelligence-techniques-in-the-petrole/15367864 (accessed November 24, 2020).

[169] D.A. Haddad, Iraqi J. of Polymers Vol.18 , No.1, 33-44 , 2015, (2015) 13.

[170] I.-C. Yeh, Modeling of strength of high-performance concrete using artificial neural networks, Cement and Concrete Research. 28 (1998) 1797-1808. https://doi.org/10.1016/S0008-8846(98)001653.

[171] C. Negro, A. Alonso, A. Blanco, J. Tijero, Breaking load and bending strength prediction in manufacture of fibre cement composites using artificial neural networks and a flocculation sensor, Composites Part A: Applied Science and Manufacturing. 36 (2005) 1617-1626. https://doi.org/10.1016/j.compositesa.2005.04.008.

[172] M. Hayajneh, A.M. Hassan, A. Alrashdan, A.T. Mayyas, Prediction of tribological behavior of aluminum-copper based composite using artificial neural network, Journal of Alloys and Compounds. 470 (2009) 584-588. https://doi.org/10.1016/j.jallcom.2008.03.035.

[173] M.A.S. Matos, S.T. Pinho, V.L. Tagarielli, Application of machine learning to predict the multiaxial strain-sensing response of CNT-polymer composites, Carbon. 146 (2019) 265-275. https://doi.org/10.1016/j.carbon.2019.02.001.

[174] I. Argatov, Artificial Neural Networks (ANNs) as a Novel Modeling Technique in Tribology, Front. Mech. Eng. 5 (2019). https://doi.org/10.3389/fmech.2019.00030. 
[175] B.A. Shuvho, M.A. Chowdhury, U.K. Debnath, Analysis of Artificial Neural Network for Predicting Erosive Wear of Nylon-12 Polymer, MPC. 8 (2019) 288-300. https://doi.org/10.1520/MPC20180164.

[176] N. Li, J.Y. Sheikh-Ahmad, A. El-Sinawi, V. Krishnaraj, Multi-objective optimization of the trimming operation of CFRPs using sensor-fused neural networks and TOPSIS, Measurement. 132 (2019) 252-262. https://doi.org/10.1016/j.measurement.2018.09.057.

[177] F. Wang, Z. Chen, C. Wu, Y. Yang, Prediction on sound insulation properties of ultrafine glass wool mats with artificial neural networks, Applied Acoustics. 146 (2019) 164-171. https://doi.org/10.1016/j.apacoust.2018.11.018.

[178] S.K. Nayak, A. Satapathy, S. Mantry, Processing and wear response study of glass-polyester composites with waste marble dust as particulate filler, Polymer Composites. 41 (2020) 2263-2273. https://doi.org/10.1002/pc.25537.

[179] W. Mucha, W. Kuś, J.C. Viana, J.P. Nunes, Operational Load Monitoring of a Composite Panel Using Artificial Neural Networks, Sensors. 20 (2020) 2534. https://doi.org/10.3390/s20092534.

[180] P.K. Kopparthi, V.R. Kundavarapu, V.R. Dasari, V.R. Kaki, B.R. Pathakokila, Modeling of glass fiber reinforced composites for optimal mechanical properties using teaching learning based optimization and artificial neural networks, SN Appl. Sci. 2 (2019) 131. https://doi.org/10.1007/s42452-019-1837-x.

[181] C. Settgast, G. Hütter, M. Kuna, M. Abendroth, A hybrid approach to simulate the homogenized irreversible elastic-plastic deformations and damage of foams by neural networks, International Journal of Plasticity. 126 (2020) 102624. https://doi.org/10.1016/j.ijplas.2019.11.003.

[182] Z. Jiang, Z. Zhang, K. Friedrich, Prediction on wear properties of polymer composites with artificial neural networks, Composites Science and Technology. 67 (2007) 168-176. https://doi.org/10.1016/j.compscitech.2006.07.026.

[183] H. Guo, J. Pandher, M. van Tooren, S. Wang, Process Modelling of Induction Welding for Thermoplastic Composite Materials By Neural Networks, in: SAMPE 2019 - Charlotte, NC, SAMPE, 2019: pp. 1-15. https://doi.org/10.33599/nasampe/s.19.1370.

[184] A.T. Seyhan, G. Tayfur, M. Karakurt, M. Tanog`lu, Artificial neural network (ANN) prediction of compressive strength of VARTM processed polymer composites, Computational Materials Science. 34 (2005) 99-105. https://doi.org/10.1016/j.commatsci.2004.11.001.

[185] S. Farhangdoust, S. Tashakori, A. Baghalian, A. Mehrabi, I.N. Tansel, Prediction of damage location in composite plates using artificial neural network modeling, in: Sensors and Smart Structures Technologies for Civil, Mechanical, and Aerospace Systems 2019, International Society for Optics and Photonics, 2019: p. 109700I. https://doi.org/10.1117/12.2517422.

[186] H. Fazilat, M. Ghatarband, S. Mazinani, Z.A. Asadi, M.E. Shiri, M.R. Kalaee, Predicting the mechanical properties of glass fiber reinforced polymers via artificial neural network and adaptive neuro-fuzzy inference system, Computational Materials Science. 58 (2012) 31-37. https://doi.org/10.1016/j.commatsci.2012.01.012.

[187] (14) (PDF) Neural network paradigms for fatigue strength prediction of fiber-reinforced composite materials, ResearchGate. (n.d.). https://www.researchgate.net/publication/257883647_Neural_network_paradigms_for_fatigue_strength _prediction_of_fiber-reinforced_composite_materials (accessed June 25, 2020).

[188] (14) (PDF) Experimental and artificial neural network ANN investigation of bending fatigue behavior of glass fiber/polyester composite shafts, ResearchGate. (n.d.). https://www.researchgate.net/publication/323945144_Experimental_and_artificial_neural_network_A 
NN_investigation_of_bending_fatigue_behavior_of_glass_fiberpolyester_composite_shafts (accessed June 26, 2020).

[189] A.P. Vassilopoulos, E.F. Georgopoulos, V. Dionysopoulos, Artificial neural networks in spectrum fatigue life prediction of composite materials, International Journal of Fatigue. 29 (2007) 2029. https://doi.org/10.1016/j.ijfatigue.2006.03.004.

[190] P. Ramasamy, S. Sampathkumar, Prediction of impact damage tolerance of drop impacted WGFRP composite by artificial neural network using acoustic emission parameters, Composites Part B: Engineering. 60 (2014) 457-462. https://doi.org/10.1016/j.compositesb.2013.12.028.

[191] S.-K. Oh, W. Pedrycz, B.-J. Park, Polynomial neural networks architecture: analysis and design, Computers \& Electrical Engineering. 29 (2003) 703-725. https://doi.org/10.1016/S00457906(02)00045-9.

[192] Y. Al-Assaf, H.E. Kadi, Fatigue life prediction of composite materials using polynomial classifiers and recurrent neural networks, Composite Structures. 77 (2007) 561-569. https://doi.org/10.1016/j.compstruct.2005.08.012.

[193] R.R. Kumar, T. Mukhopadhya, K.M. Pandey, S. Dey, Chapter 5 - Prediction capability of polynomial neural network for uncertain buckling behavior of sandwich plates, in: P. Samui, D. Tien Bui, S. Chakraborty, R.C. Deo (Eds.), Handbook of Probabilistic Models, Butterworth-Heinemann, 2020: pp. 131-140. https://doi.org/10.1016/B978-0-12-816514-0.00005-9.

[194] S.-C.B. Lo, H.-P. Chan, J.-S. Lin, H. Li, M.T. Freedman, S.K. Mun, Artificial convolution neural network for medical image pattern recognition, Neural Networks. 8 (1995) 1201-1214. https://doi.org/10.1016/0893-6080(95)00061-5.

[195] S. Feng, H. Zhou, H. Dong, Using deep neural network with small dataset to predict material defects, Materials \& Design. 162 (2019) 300-310. https://doi.org/10.1016/j.matdes.2018.11.060.

[196] S.-C.B. Lo, S.-L.A. Lou, Jyh-Shyan Lin, M.T. Freedman, M.V. Chien, S.K. Mun, Artificial convolution neural network techniques and applications for lung nodule detection, IEEE Transactions on Medical Imaging. 14 (1995) 711-718. https://doi.org/10.1109/42.476112.

[197] D.W. Abueidda, M. Almasri, R. Ammourah, U. Ravaioli, I.M. Jasiuk, N.A. Sobh, Prediction and optimization of mechanical properties of composites using convolutional neural networks, Composite Structures. 227 (2019) 111264. https://doi.org/10.1016/j.compstruct.2019.111264.

[198] L.A. Miccio, G.A. Schwartz, From chemical structure to quantitative polymer properties prediction through convolutional neural networks, Polymer. $193 \quad$ (2020) 122341. https://doi.org/10.1016/j.polymer.2020.122341.

[199] S.R. Karnik, V.N. Gaitonde, J.C. Rubio, A.E. Correia, A.M. Abrão, J.P. Davim, Delamination analysis in high speed drilling of carbon fiber reinforced plastics (CFRP) using artificial neural network model, Materials \& Design. 29 (2008) 1768-1776. https://doi.org/10.1016/j.matdes.2008.03.014.

[200] Z. Zhang, K. Friedrich, K. Velten, Prediction on tribological properties of short fibre composites using artificial neural networks, Wear. 252 (2002) 668-675. https://doi.org/10.1016/S00431648(02)00023-6.

[201] X. LiuJie, J.P. Davim, R. Cardoso, Prediction on tribological behaviour of composite PEEKCF30 using artificial neural networks, Journal of Materials Processing Technology. 189 (2007) 374378. https://doi.org/10.1016/j.jmatprotec.2007.02.019.

[202] K. Yanamandra, G.L. Chen, X. Xu, G. Mac, N. Gupta, Reverse engineering of additive manufactured composite part by toolpath reconstruction using imaging and machine learning,

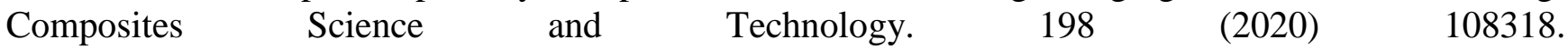
https://doi.org/10.1016/j.compscitech.2020.108318. 
[203] M.H. Modarres, R. Aversa, S. Cozzini, R. Ciancio, A. Leto, G.P. Brandino, Neural Network for Nanoscience Scanning Electron Microscope Image Recognition, Scientific Reports. 7 (2017) 13282. https://doi.org/10.1038/s41598-017-13565-z.

[204] Ö. Erkan, B. Işık, A. Çiçek, F. Kara, Prediction of Damage Factor in end Milling of Glass Fibre Reinforced Plastic Composites Using Artificial Neural Network, Appl Compos Mater. 20 (2013) 517536. https://doi.org/10.1007/s10443-012-9286-3.

[205] D. Sammons, W.P. Winfree, E. Burke, S. Ji, Segmenting delaminations in carbon fiber reinforced polymer composite CT using convolutional neural networks, in: Minneapolis, Minnesota, 2016: p. 110014. https://doi.org/10.1063/1.4940585.

[206] J.P. McCrory, S.Kh. Al-Jumaili, D. Crivelli, M.R. Pearson, M.J. Eaton, C.A. Featherston, M. Guagliano, K.M. Holford, R. Pullin, Damage classification in carbon fibre composites using acoustic emission: A comparison of three techniques, Composites Part B: Engineering. 68 (2015) 424-430. https://doi.org/10.1016/j.compositesb.2014.08.046.

[207] A. Sharma, A.K. Subramaniyan, V. Kushvaha, Effect of aspect ratio on dynamic fracture toughness of particulate polymer composite using artificial neural network, Engineering Fracture Mechanics. 228 (2020) 106907. https://doi.org/10.1016/j.engfracmech.2020.106907.

[208] V. Kushvaha, S.A. Kumar, P. Madhushri, A. Sharma, Artificial neural network technique to predict dynamic fracture of particulate composite, Journal of Composite Materials. (2020) 0021998320911418. https://doi.org/10.1177/0021998320911418.

[209] D.-W. Lee, S.-H. Hong, S.-S. Cho, W.-S. Joo, A study on fatigue damage modeling using neural networks, J Mech Sci Technol. 19 (2005) 1393-1404. https://doi.org/10.1007/BF03023898.

[210] P.R. Pati, Prediction and wear performance of red brick dust filled glass-epoxy composites using neural networks, Int J Plast Technol. 23 (2019) 253-260. https://doi.org/10.1007/s12588-01909257-0.

[211] S.S. Mahapatra, A Grey-based Taguchi Method for Wear Assesment of Red Mud Filled Polyester Composites, IJMO. (2011) 80-88. https://doi.org/10.7763/IJMO.2011.V1.15.

[212] J. Zhu, Y. Shi, X. Feng, H. Wang, X. Lu, Prediction on tribological properties of carbon fiber and $\mathrm{TiO} 2$ synergistic reinforced polytetrafluoroethylene composites with artificial neural networks, Materials \& Design. 30 (2009) 1042-1049. https://doi.org/10.1016/j.matdes.2008.06.045.

[213] K. Velten, R. Reinicke, K. Friedrich, Wear volume prediction with artificial neural networks, Tribology International. 33 (2000) 731-736. https://doi.org/10.1016/S0301-679X(00)00115-8.

[214] S.P. Jones, R. Jansen, R.L. Fusaro, Preliminary Investigation of Neural Network Techniques to Predict Tribological Properties, Tribology Transactions. $40 \quad$ (1997) 312-320. https://doi.org/10.1080/10402009708983660.

[215] K.M. Hamdia, T. Lahmer, T. Nguyen-Thoi, T. Rabczuk, Predicting the fracture toughness of PNCs: A stochastic approach based on ANN and ANFIS, Computational Materials Science. 102 (2015) 304-313. https://doi.org/10.1016/j.commatsci.2015.02.045.

[216] V. Bheemreddy, K. Chandrashekhara, L.R. Dharani, G.E. Hilmas, Modeling of fiber pull-out in continuous fiber reinforced ceramic composites using finite element method and artificial neural networks, $\quad$ Computational $\quad$ Materials $\quad$ Science. $\quad 79 \quad$ 663-673. https://doi.org/10.1016/j.commatsci.2013.07.026.

[217] L.A. Gyurova, P. Miniño-Justel, A.K. Schlarb, Modeling the sliding wear and friction properties of polyphenylene sulfide composites using artificial neural networks, Wear. 268 (2010) 708714. https://doi.org/10.1016/j.wear.2009.11.008. 
[218] C. Leone, G. Caprino, I. de Iorio, Interpreting acoustic emission signals by artificial neural networks to predict the residual strength of pre-fatigued GFRP laminates, Composites Science and Technology. 66 (2006) 233-239. https://doi.org/10.1016/j.compscitech.2005.04.032.

[219] M.V. Mousavi, H. Khoramishad, The effect of hybridization on high-velocity impact response of carbon fiber-reinforced polymer composites using finite element modeling, Taguchi method and artificial neural network, Aerospace Science and Technology. 94 (2019) 105393. https://doi.org/10.1016/j.ast.2019.105393.

[220] S.M. Khan, S.A. Malik, N. Gull, S. Saleemi, A. Islam, M.T.Z. Butt, Fabrication and modelling of the macro-mechanical properties of cross-ply laminated fibre-reinforced polymer composites using artificial neural network, Advanced Composite Materials. 28 (2019) 409-423. https://doi.org/10.1080/09243046.2019.1573448.

[221] M.S. Kabbani, H.A. El Kadi, Predicting the effect of cooling rate on the mechanical properties of glass fiber-polypropylene composites using artificial neural networks, Journal of Thermoplastic Composite Materials. 32 (2019) 1268-1281. https://doi.org/10.1177/0892705718792351.

[222] R. Haddad, M. Haddad, Predicting fiber-reinforced polymer-concrete bond strength using artificial neural networks: A comparative analysis study, Structural Concrete. n/a (n.d.). https://doi.org/10.1002/suco.201900298.

[223] M.-K. Kazi, F. Eljack, E. Mahdi, Optimal filler content for cotton fiber/PP composite based on mechanical properties using artificial neural network, Composite Structures. 251 (2020) 112654. https://doi.org/10.1016/j.compstruct.2020.112654.

[224] S. Mahalingam, V. Gopalan, H. Velivela, V. Pragasam, P. Prabhakaran, V. Suthenthiraveerappa, Studies on shear strength of CNT/coir fibre/fly ash-reinforced epoxy polymer composites, Emerging Materials Research. 9 (2020) 78-88. https://doi.org/10.1680/jemmr.19.00098.

[225] A. Solati, M. Hamedi, M. Safarabadi, Combined GA-ANN approach for prediction of HAZ and bearing strength in laser drilling of GFRP composite, Optics \& Laser Technology. 113 (2019) 104115. https://doi.org/10.1016/j.optlastec.2018.12.016.

[226] S.K. Antil, P. Antil, S. Singh, A. Kumar, C.I. Pruncu, Artificial Neural Network and Response Surface Methodology Based Analysis on Solid Particle Erosion Behavior of Polymer Matrix Composites, Materials. 13 (2020) 1381. https://doi.org/10.3390/ma13061381.

[227] A. Barbosa, P. Upadhyaya, E. Iype, Neural network for mechanical property estimation of multilayered laminate composite, Materials Today: Proceedings. 28 (2020) 982-985. https://doi.org/10.1016/j.matpr.2019.12.336.

[228] C. Qian, Y. Ran, J. He, Y. Ren, B. Sun, W. Zhang, R. Wang, Application of artificial neural networks for quantitative damage detection in unidirectional composite structures based on Lamb waves, Advances in Mechanical Engineering. $12 \quad$ (2020) 168781402091473. https://doi.org/10.1177/1687814020914732.

[229] K. Sumesh, K. Kanthavel, Optimizing various parameters influencing mechanical properties of banana/coir natural fiber composites using grey relational analysis and artificial neural network models, Journal of Industrial Textiles. (2020) 1528083720930304. https://doi.org/10.1177/1528083720930304.

[230] O.T. Adesina, T. Jamiru, I.A. Daniyan, E.R. Sadiku, O.F. Ogunbiyi, O.S. Adesina, L.W. Beneke, Mechanical property prediction of SPS processed GNP/PLA polymer nanocomposite using $\begin{array}{lllllll}\text { artificial neural network, Cogent } & \text { Engineering. } & 7 & \text { (2020) } & 1720894 .\end{array}$ https://doi.org/10.1080/23311916.2020.1720894.

[231] A. Jain, B. Singh, Y. Shrivastava, Identification of Safe Machining Range for Laser Drilling of Basalt-Glass Hybrid Composite Using Artificial Neural Network, in: S. Yadav, D.B. Singh, P.K. Arora, H. Kumar (Eds.), Proceedings of International Conference in Mechanical and Energy 
Technology: ICMET 2019, India, Springer, Singapore, 2020: pp. 767-775. https://doi.org/10.1007/978981-15-2647-3_72.

[232] M.A. Omari, A. Almagableh, I. Sevostianov, M.S. Ashhab, A.B. Yaseen, Modeling of the viscoelastic properties of thermoset vinyl ester nanocomposite using artificial neural network, $\begin{array}{lllllll}\text { International Journal of } & \text { Engineering } & \text { Science. } & 150 & \text { (2020) }\end{array}$ https://doi.org/10.1016/j.ijengsci.2020.103242.

[233] S. Yan, X. Zou, M. Ilkhani, A. Jones, An efficient multiscale surrogate modelling framework for composite materials considering progressive damage based on artificial neural networks, $\begin{array}{llllll}\text { Composites } & \text { Part } & \text { B: } & \text { Engineering. } & 194 & \text { (2020) }\end{array}$ https://doi.org/10.1016/j.compositesb.2020.108014.

[234] S.K. Kamane, N.K. Patil, B.R. Patagundi, Use of artificial neural network to predict the bending behavior of steel I beam externally attached with FRP sheets, Materials Today: Proceedings. (2020). https://doi.org/10.1016/j.matpr.2020.04.893.

[235] L. Wu, K. Zulueta, Z. Major, A. Arriaga, L. Noels, Bayesian inference of non-linear multiscale model parameters accelerated by a Deep Neural Network, Computer Methods in Applied Mechanics and Engineering. 360 (2020) 112693. https://doi.org/10.1016/j.cma.2019.112693.

[236] T. Sabiston, K. Inal, P. Lee-Sullivan, Application of Artificial Neural Networks to predict fibre orientation in long fibre compression moulded composite materials, Composites Science and Technology. 190 (2020) 108034. https://doi.org/10.1016/j.compscitech.2020.108034.

[237] G. Ciaburro, G. Iannace, J. Passaro, A. Bifulco, D. Marano, M. Guida, F. Marulo, F. Branda, Artificial neural network-based models for predicting the sound absorption coefficient of electrospun poly(vinyl pyrrolidone)/silica composite, Applied Acoustics. $169 \quad$ (2020) 107472. https://doi.org/10.1016/j.apacoust.2020.107472.

[238] H. Zhou, H. Liu, T. Kuang, Q. Jiang, Z. Chen, W. Li, Optimization of Residual Wall Thickness Uniformity in Short-Fiber-Reinforced Composites Water-Assisted Injection Molding Using Response Surface Methodology and Artificial Neural Network-Genetic Algorithm, Advances in Polymer Technology. 2020 (2020) e6154694. https://doi.org/10.1155/2020/6154694.

[239] M. Zakaulla, F. Parveen, Amreen, Harish, N. Ahmad, Artificial neural network based prediction on tribological properties of polycarbonate composites reinforced with graphene and boron carbide particle, Materials $\quad$ Today: $\quad$ Proceedings. $26 \quad$ (2020) 2964. https://doi.org/10.1016/j.matpr.2019.11.276.

[240] B.P. Nanda, A. Satapathy, An Analysis of the Sliding Wear Characteristics of Epoxy-Based Hybrid Composites Using Response Surface Method and Neural Computation, Journal of Natural Fibers. 0 (2020) 1-15. https://doi.org/10.1080/15440478.2020.1722781.

[241] B.R.S. Reddy, M. Premasudha, B.B. Panigrahi, K.-K. Cho, N.G.S. Reddy, Modeling constituent-property relationship of polyvinylchloride composites by neural networks, Polymer Composites. n/a (n.d.). https://doi.org/10.1002/pc.25612.

[242] Z. Wang, P. Dixit, F. Chegdani, B. Takabi, B.L. Tai, M.E. Mansori, S. Bukkapatnam, Bidirectional Gated Recurrent Deep Learning Neural Networks for Smart Acoustic Emission Sensing of Natural Fiber-Reinforced Polymer Composite Machining Process, 4 (2020). https://doi.org/10.1520/SSMS20190042.

[243] M.A.S. Matos, S.T. Pinho, V.L. Tagarielli, Predictions of the electrical conductivity of composites of polymers and carbon nanotubes by an artificial neural network, Scripta Materialia. 166 (2019) 117-121. https://doi.org/10.1016/j.scriptamat.2019.03.003.

[244] Y. Wang, M. Zhang, A. Lin, A. Iyer, A. Shanker Prasad, X. Li, Y. Zhang, L. S. Schadler, W. Chen, L. Catherine Brinson, Mining structure-property relationships in polymer nanocomposites using 
data driven finite element analysis and multi-task convolutional neural networks, Molecular Systems Design \& Engineering. 5 (2020) 962-975. https://doi.org/10.1039/D0ME00020E.

[245] X. Lu, D.G. Giovanis, J. Yvonnet, V. Papadopoulos, F. Detrez, J. Bai, A data-driven computational homogenization method based on neural networks for the nonlinear anisotropic electrical response of graphene/polymer nanocomposites, Comput Mech. 64 (2019) 307-321. https://doi.org/10.1007/s00466-018-1643-0.

[246] H.H. Parikh, P.P. Gohil, 13 - Experimental determination of tribo behavior of fiber-reinforced composites and its prediction with artificial neural networks, in: M. Jawaid, M. Thariq, N. Saba (Eds.), Durability and Life Prediction in Biocomposites, Fibre-Reinforced Composites and Hybrid Composites, Woodhead Publishing, 2019: pp. 301-320. https://doi.org/10.1016/B978-0-08-1022900.00013-1.

[247] D.S. Kumar, M. Rajmohan, Optimizing Wear Behavior of Epoxy Composites Using Response Surface Methodology and Artificial Neural Networks, Polymer Composites. 40 (2019) 2812-2818. https://doi.org/10.1002/pc.25089.

[248] J. Farahbakhsh, M. Delnavaz, V. Vatanpour, Simulation and characterization of novel reverse osmosis membrane prepared by blending polypyrrole coated multiwalled carbon nanotubes for brackish water desalination and antifouling properties using artificial neural networks, Journal of Membrane Science. 581 (2019) 123-138. https://doi.org/10.1016/j.memsci.2019.03.050.

[249] F. Robbany, B. Pramujati, Suhardjono, M.K. Effendi, B.O.P. Soepangkat, R. Norcahyo, Multi response prediction of cutting force and delamination in carbon fiber reinforced polymer using backpropagation neural network-genetic algorithm, AIP Conference Proceedings. 2114 (2019) 030012. https://doi.org/10.1063/1.5112416.

[250] Maryam Ataeefard, Y. Mohammadi, M.R. Saeb, Intelligently Synthesized In Situ Suspension Carbon Black/Styrene/Butylacrylate Composites: Using Artificial Neural Networks towards Printing Inks with Well-Controlled Properties, Polym. Sci. Ser. A. 61 (2019) 667-680. https://doi.org/10.1134/S0965545X19050031.

[251] I. Kopal, J. Vršková, D. Ondrušová, M. Harničárová, J. Valíček, Z. Koleničová, Modeling the thermal decomposition of friction composite systems based on yarn reinforced polymer matrices using artificial neural networks, Materialwissenschaft Und Werkstofftechnik. 50 (2019) 616-628. https://doi.org/10.1002/mawe.201800178.

[252] J. Zhou, Y. Li, D. Li, Y. Wen, Online learning based intelligent temperature control during polymer composites microwave curing process, Chemical Engineering Journal. 370 (2019) 455-465. https://doi.org/10.1016/j.cej.2019.03.204.

[253] G.S. Zeng, C. Hu, S. Zou, L. Zhang, G. Sun, BP neural network model for predicting the mechanical performance of a foamed wood-fiber reinforced thermoplastic starch composite, Polymer Composites. 40 (2019) 3923-3928. https://doi.org/10.1002/pc.25252.

[254] Liang yang, Dongsheng zhang, Xining zhang, Aifen tian, Prediction of the Actuation Property of $\mathrm{Cu}$ Ionic Polymer-Metal Composites Based on Backpropagation Neural Networks, ACS Publications. (2020). https://doi.org/10.1021/acsomega.9b03725.

[255] A. Doblies, B. Boll, B. Fiedler, Prediction of Thermal Exposure and Mechanical Behavior of Epoxy Resin Using Artificial Neural Networks and Fourier Transform Infrared Spectroscopy, Polymers. 11 (2019) 363. https://doi.org/10.3390/polym11020363.

[256] B.O.P. Soepangkat, M.K. Effendi, B. Pramujati, R. Norcahyo, F. Robbany, Delamination factor and cutting force optimizations in end-milling of carbon fiber reinforced polymer composites using backpropagation neural network-ant colony optimization, AIP Conference Proceedings. 2187 (2019) 030010. https://doi.org/10.1063/1.5138314. 
[257] N. Saeed, H. Al Zarkani, M.A. Omar, Sensitivity and Robustness of Neural Networks for Defect-Depth Estimation in CFRP Composites, J Nondestruct Eval. 38 (2019) 74. https://doi.org/10.1007/s10921-019-0607-4.

[258] P.R. Pati, A. Satapathy, A Study on Tribological Behavior of Linz-Donawitz Slag Filled Polypropylene Composites Using Experimental Design and Neural Networks, in: American Society of Mechanical Engineers Digital Collection, 2018. https://doi.org/10.1115/GTINDIA2017-4514.

[259] Upendra K.Mallela, Akhil Upadhyay, Buckling load prediction of laminated composite stiffened panels subjected to in-plane shear using artificial neural networks, Elsevier. 102 (2016) 158164. https://doi.org/10.1016/j.tws.2016.01.025.

[260] Insha Wani, Himanshu Kumar, Sanjay M R, Lin Peng, Suchart Siengchin, Vinod Kushvaha, A Multi Regression Model for Predicting the soil cracks in Pig Manure and Wood Biochar Amended soils, Journal of Hazardous, Toxic, and Radioactive Waste. (2020). https://doi.org/10.1061/(ASCE)HZ.2153-5515.0000561.

[261] C.M. Bishop, Pattern recognition and machine learning, CERN Document Server. (2006). https://cds.cern.ch/record/998831 (accessed July 31, 2020).

[262] M. Tirzïte, M. Bukovskis, G. Strazda, N. Jurka, I. Taivans, Detection of lung cancer with electronic nose and logistic regression analysis, J. Breath Res. 13 (2018) 016006. https://doi.org/10.1088/1752-7163/aae1b8.

[263] Hybrid decision tree and logistic regression classifier for email spam detection - IEEE Conference Publication,

(n.d.). https://ieeexplore.ieee.org/abstract/document/7863267? casa_token=daFvJvH2fjEAAAAA:usx8M8Ts6 Se6fBoPTtrC7amta89jvFt4oz0_2wQqhQ9vRX0gR59rbkj1ShYUms7kzzGSMRnZKe0T7Q (accessed November 29, 2020).

[264] C. Zhu, C.U. Idemudia, W. Feng, Improved logistic regression model for diabetes prediction by integrating PCA and K-means techniques, Informatics in Medicine Unlocked. 17 (2019) 100179. https://doi.org/10.1016/j.imu.2019.100179.

[265] R.L. Sakaguchi, B.D. Wiltbank, C.F. Murchison, Contraction force rate of polymer composites is linearly correlated with irradiance, Dental Materials. 20 (2004) 402-407. https://doi.org/10.1016/j.dental.2003.11.004.

[266] M. Noryani, S.M. Sapuan, M.T. Mastura, M.Y.M. Zuhri, E.S. Zainudin, A Statistical Framework for Selecting Natural Fibre Reinforced Polymer Composites Based on Regression Model, Fibers Polym. 19 (2018) 1039-1049. https://doi.org/10.1007/s12221-018-8113-3.

[267] S.G. Chen, J.W. Hu, M.Q. Zhang, M.Z. Rong, Effects of temperature and vapor pressure on the gas sensing behavior of carbon black filled polyurethane composites, Sensors and Actuators B: Chemical. 105 (2005) 187-193. https://doi.org/10.1016/j.snb.2004.05.060.

[268] Polymerization shrinkage and contraction stress of dental resin composites, Dental Materials. 21 (2005) 1150-1157. https://doi.org/10.1016/j.dental.2005.02.004.

[269] (17) (PDF) Deconvolution of ultrasonic echoes using Bernoulli-Gaussian processes for composite materials inspection, ResearchGate. (n.d.). https://www.researchgate.net/publication/43965433_Deconvolution_of_ultrasonic_echoes_using_Bern oulli-Gaussian_processes_for_composite_materials_inspection (accessed July 31, 2020).

[270] D. Xu, P.F. Liu, Z.P. Chen, J.X. Leng, L. Jiao, Achieving robust damage mode identification of adhesive composite joints for wind turbine blade using acoustic emission and machine learning, Composite Structures. 236 (2020) 111840. https://doi.org/10.1016/j.compstruct.2019.111840. 
[271] X. Chen, F. Kopsaftopoulos, Q. Wu, H. Ren, F.-K. Chang, Flight State Identification of a SelfSensing Wing via an Improved Feature Selection Method and Machine Learning Approaches, Sensors. 18 (2018) 1379. https://doi.org/10.3390/s18051379.

[272] M.R.P. Elenchezhian, A. Nandini, V. Vadlamudi, R. Raihan, K. Reifsnider, Detection and Prediction of Defects in Composite Materials Using Di-Electric Characterization and Neural Networks, (2018). https://rc.library.uta.edu/uta-ir/handle/10106/27498 (accessed August 1, 2020).

[273] R. Cao, S. Naya, R. Artiaga, A. García, A. Varela, Logistic approach to polymer degradation in dynamic TGA, Polymer Degradation and Stability. 85 (2004) 667-674. https://doi.org/10.1016/j.polymdegradstab.2004.03.006.

[274] O. Folorunso, Y. Hamam, R. Sadiku, S.S. Ray, A.G. Joseph, Parametric Analysis of Electrical $\begin{array}{llllll}\text { Conductivity of } & \text { Polymer-Composites, } & \text { Polymers. } & 11 & \text { (2019) } & 1250 .\end{array}$ https://doi.org/10.3390/polym11081250.

[275] T.L.L. Berge, G.B. Lygre, S.A. Lie, L. Björkman, Polymer-based dental filling materials placed during pregnancy and risk to the foetus, BMC Oral Health. 18 (2018) 144. https://doi.org/10.1186/s12903-018-0608-1.

[276] V.-S. Osburg, M. Strack, W. Toporowski, Consumer acceptance of Wood-Polymer Composites: a conjoint analytical approach with a focus on innovative and environmentally concerned consumers, Journal of Cleaner Production. 110 (2016) 180-190. https://doi.org/10.1016/j.jclepro.2015.04.086.

[277] J.B. Henderson, T.E. Wiecek, A Mathematical Model to Predict the Thermal Response of Decomposing, Expanding Polymer Composites, Journal of Composite Materials. 21 (1987) 373-393. https://doi.org/10.1177/002199838702100406.

[278] D.A. Gerdolle, E. Mortier, D. Droz, Microleakage and Polymerization Shrinkage of Various Polymer Restorative Materials, Journal of Dentistry for Children. 75 (2008) 125-133.

[279] C.E. Rasmussen, Gaussian Processes in Machine Learning, in: O. Bousquet, U. von Luxburg, G. Rätsch (Eds.), Advanced Lectures on Machine Learning: ML Summer Schools 2003, Canberra, Australia, February 2 - 14, 2003, Tübingen, Germany, August 4 - 16, 2003, Revised Lectures, Springer, Berlin, Heidelberg, 2004: pp. 63-71. https://doi.org/10.1007/978-3-540-28650-9_4.

[280] Jerome sacks, William J. Welch, Toby J. Mitchell, Henry P Wynn, Design and analysis of computer experiments, Statistical Science. 4 (1989) 409-423.

[281] Adaptive Sampling of Ocean Processes Using an AUV with a Gaussian Proxy Model, IFACPapersOnLine. 51 (2018) 238-243. https://doi.org/10.1016/j.ifacol.2018.09.509.

[282] I. Rychlik, P. Johannesson, M.R. Leadbetter, Modelling and Statistical Analysis of ocean-wave data using transformed gaussian processes, Marine Structures. 10 (1997) 13-47. https://doi.org/10.1016/S0951-8339(96)00017-2.

[283] G. Jona-Lasinio, A. Gelfand, M. Jona-Lasinio, Spatial analysis of wave direction data using wrapped Gaussian processes, Ann. Appl. Stat. 6 (2012) 1478-1498. https://doi.org/10.1214/12AOAS576.

[284] K. Blix, G. Camps-Valls, R. Jenssen, Gaussian Process Sensitivity Analysis for Oceanic Chlorophyll Estimation, IEEE J. Sel. Top. Appl. Earth Observations Remote Sensing. 10 (2017) 12651277. https://doi.org/10.1109/JSTARS.2016.2641583.

[285] Z. Wang, W. Xing, R. Kirby, S. Zhe, Physics Regularized Gaussian Processes, ArXiv:2006.04976 [Cs, Stat]. (2020). http://arxiv.org/abs/2006.04976 (accessed July 28, 2020).

[286] C.E. Rasmussen, H. Nickisch, Gaussian Processes for Machine Learning (GPML) Toolbox, (n.d.) 5 . 
[287] L. Meng, J. Zhang, Process Design of Laser Powder Bed Fusion of Stainless Steel Using a Gaussian Process-Based Machine Learning Model, JOM. 72 (2020) 420-428. https://doi.org/10.1007/s11837-019-03792-2.

[288] C. Linkletter, D. Bingham, N. Hengartner, D. Higdon, K.Q. Ye, Variable Selection for Gaussian Process Models in Computer Experiments, Technometrics. 48 (2006) 478-490. https://doi.org/10.1198/004017006000000228.

[289] M. Seeger, Gaussian processes for machine learning, Int. J. Neur. Syst. 14 (2004) 69-106. https://doi.org/10.1142/S0129065704001899.

[290] C.-T. Chen, G.X. Gu, Machine learning for composite materials, MRS Communications. 9 (2019) 556-566. https://doi.org/10.1557/mrc.2019.32.

[291] E. Snelson, Z. Ghahramani, Sparse Gaussian Processes using Pseudo-inputs, in: Y. Weiss, B. Schölkopf, J.C. Platt (Eds.), Advances in Neural Information Processing Systems 18, MIT Press, 2006: pp. 1257-1264. http://papers.nips.cc/paper/2857-sparse-gaussian-processes-using-pseudo-inputs.pdf (accessed July 29, 2020).

[292] Y. Liu, S. Mohanty, A. Chattopadhyay, A Gaussian process based prognostics framework for composite structures, in: Modeling, Signal Processing, and Control for Smart Structures 2009, International Society for Optics and Photonics, 2009: p. 72860J. https://doi.org/10.1117/12.815889.

[293] (14) Gaussian process regression of chirplet decomposed ultrasonic B-scans of a simulated design case $\quad$ Request PDF, ResearchGate. (n.d.). https://www.researchgate.net/publication/324664656_Gaussian_process_regression_of_chirplet_decom posed_ultrasonic_B-scans_of_a_simulated_design_case (accessed July 30, 2020).

[294] Y. Wang, Y. Zhang, H. Zhao, X. Li, Y. Huang, L.S. Schadler, W. Chen, L.C. Brinson, Identifying interphase properties in polymer nanocomposites using adaptive optimization, Composites Science and Technology. 162 (2018) 146-155. https://doi.org/10.1016/j.compscitech.2018.04.017.

[295] (14) Reliability of surface response to excitation method for data-driven prognostics using Gaussian process regression | Request PDF, (n.d.). https://www.researchgate.net/publication/324048785_Reliability_of_surface_response_to_excitation_ method_for_data-driven_prognostics_using_Gaussian_process_regression (accessed July 30, 2020).

[296] N.K. Hansoge, T. Huang, R. Sinko, W. Xia, W. Chen, S. Keten, Materials by Design for Stiff and Tough Hairy Nanoparticle Assemblies, ACS Nano. 12 (2018) 7946-7958. https://doi.org/10.1021/acsnano.8b02454.

[297] F. Qin, H.X. Peng, C. Prunier, C. Brosseau, Mechanical-electromagnetic coupling of microwire polymer composites at microwave frequencies, Appl. Phys. Lett. 97 (2010) 153502. https://doi.org/10.1063/1.3502488.

[298] F.A. Hermawati, I.M. Kastiawan, Muhyin, Digital Microscopy Image Enhancement Technique for Microstructure Image Analysis of Bottom Ash Particle Polymer Composites, in: I.A. Parinov, S.-H. Chang, B.T. Long (Eds.), Advanced Materials, Springer International Publishing, Cham, 2020: pp. 235-244. https://doi.org/10.1007/978-3-030-45120-2_20.

[299] L.S. Schadler, W. Chen, L.C. Brinson, R. Sundararaman, P. Gupta, P. Prabhune, A. Iyer, Y. Wang, A. Shandilya, A perspective on the data-driven design of polymer nanodielectrics, J. Phys. D: Appl. Phys. 53 (2020) 333001. https://doi.org/10.1088/1361-6463/ab8b01.

[300] X. Li, R. Tao, A. Yudhanto, G. Lubineau, How the spatial correlation in adhesion properties influences the performance of secondary bonding of laminated composites, International Journal of Solids and Structures. 196-197 (2020) 41-52. https://doi.org/10.1016/j.ijsolstr.2020.04.012. 
[301] V. Ilic, J. Tadić, A. Imširagić, Kriging with machine learning covariates in environmental sciences: A hybrid approach, 2016.

[302] G. Lupera, A. Shokry, S. Medina-González, E. Vyhmeister, A. Espuña, Ordinary Kriging: A machine learning tool applied to mixed-integer multiparametric approach, in: A. Friedl, J.J. Klemeš, S. Radl, P.S. Varbanov, T. Wallek (Eds.), Computer Aided Chemical Engineering, Elsevier, 2018: pp. 531-536. https://doi.org/10.1016/B978-0-444-64235-6.50094-2.

[303] J. Chen, S. Mak, V.R. Joseph, C. Zhang, Adaptive design for Gaussian process regression under censoring, ArXiv:1910.05452 [Stat]. (2019). http://arxiv.org/abs/1910.05452 (accessed July 31, 2020).

[304] G. Iannace, G. Ciaburro, Modelling sound absorption properties for recycled polyethylene terephthalate-based material using Gaussian regression, Building Acoustics. (2020) 1351010X20933132. https://doi.org/10.1177/1351010X20933132.

[305] P. Inguva, L. Mason, I. Pan, M. Hengardi, O.K. Matar, Numerical simulation, clustering and prediction of multi-component polymer precipitation, ArXiv:2007.07276 [Cond-Mat, Physics:Physics, Stat]. (2020). http://arxiv.org/abs/2007.07276 (accessed July 31, 2020).

[306] Z. Ma, W. Zhang, Z. Luo, X. Sun, Z. Li, L. Lin, Ultrasonic characterization of thermal barrier coatings porosity through BP neural network optimizing Gaussian process regression algorithm, Ultrasonics. 100 (2020) 105981. https://doi.org/10.1016/j.ultras.2019.105981.

[307] C.B. Davis, C.M. Hans, T.J. Santner, Prediction Using a Bayesian Heteroscedastic Composite Gaussian Process, ArXiv:1906.10737 [Stat]. (2019). http://arxiv.org/abs/1906.10737 (accessed July 31, 2020).

[308] A. Iyer, Y. Zhang, A. Prasad, S. Tao, Y. Wang, L. Schadler, L.C. Brinson, W. Chen, DataCentric Mixed-Variable Bayesian Optimization for Materials Design, in: American Society of Mechanical Engineers Digital Collection, 2019. https://doi.org/10.1115/DETC2019-98222.

[309] P. Fernandez-Zelaia, Y.C. Yabansu, S.R. Kalidindi, A Comparative Study of the Efficacy of Local/Global and Parametric/Nonparametric Machine Learning Methods for Establishing StructureProperty Linkages in High-Contrast 3D Elastic Composites, Integr Mater Manuf Innov. 8 (2019) 6781. https://doi.org/10.1007/s40192-019-00129-4.

[310] H. Shi, S. Yuan, Z. Li, H. Song, J. Qian, Evaluation of surface roughness based on sampling array for rotary ultrasonic machining of carbon fiber reinforced polymer composites, Measurement. 138 (2019) 175-181. https://doi.org/10.1016/j.measurement.2019.02.002.

[311] J.D. Carrico, T. Hermans, K.J. Kim, K.K. Leang, 3D-Printing and Machine Learning Control of Soft Ionic Polymer-Metal Composite Actuators, Scientific Reports. 9 (2019) 17482. https://doi.org/10.1038/s41598-019-53570-y.

[312] S. Jeong, M. Murayama, K. Yamamoto, Efficient Optimization Design Method Using Kriging Model, Journal of Aircraft. 42 (2005) 413-420. https://doi.org/10.2514/1.6386.

[313] M.A.R. Loja, J.I. Barbosa, C.M. Mota Soares, Dynamic behaviour of soft core sandwich beam structures using kriging-based layerwise models, Composite Structures. 134 (2015) 883-894. https://doi.org/10.1016/j.compstruct.2015.08.096.

[314] Z. Wang, J.H.S. Almeida Jr., L. St-Pierre, Z. Wang, S.G.P. Castro, Reliability-based buckling optimization with an accelerated Kriging metamodel for filament-wound variable angle tow composite $\begin{array}{lllll}\text { cylinders, } & \text { Composite } & \text { Structures. } & 254 & \text { (2020) }\end{array}$ https://doi.org/10.1016/j.compstruct.2020.112821.

[315] M. Slamani, J.-F. Chatelain, Kriging versus Bezier and regression methods for modeling and prediction of cutting force and surface roughness during high speed edge trimming of carbon fiber 
reinforced $\quad$ polymers, $\quad 15$ https://doi.org/10.1016/j.measurement.2019.107370.

(2020)

107370.

[316] J. Zhao, G. Cheng, Sequential kriging-based closure approximations for flow-induced fiber orientation and prediction of composite stiffness, Polymer Composites. 40 (2019) 1748-1761. https://doi.org/10.1002/pc.24930.

[317] D.-X. Su, J. Zhao, Y. Wang, M.-J. Qu, Kriging-based orthotropic closure for flow-induced fiber orientation and the part stiffness predictions with experimental investigation, Polymer Composites. 40 (2019) 3844-3856. https://doi.org/10.1002/pc.25243.

[318] A. Khan, M.H. Shamsi, T.-S. Choi, Correlating dynamical mechanical properties with temperature and clay composition of polymer-clay nanocomposites, Computational Materials Science. 45 (2009) 257-265. https://doi.org/10.1016/j.commatsci.2008.09.027.

[319] S. Suthaharan, Support Vector Machine, in: S. Suthaharan (Ed.), Machine Learning Models and Algorithms for Big Data Classification: Thinking with Examples for Effective Learning, Springer US, Boston, MA, 2016: pp. 207-235. https://doi.org/10.1007/978-1-4899-7641-3_9.

[320] P. Ding, Q. Li, X. Huang, Classification of acoustic emission sources produced by carbon/epoxy composite based on support vector machine, IOP Conf. Ser.: Mater. Sci. Eng. 87 (2015) 012002. https://doi.org/10.1088/1757-899X/87/1/012002.

[321] C. Cortes, V. Vapnik, Support-vector networks, Mach Learn. 20 (1995) 273-297. https://doi.org/10.1007/BF00994018.

[322] Learning with Kernels | Guide books, (n.d.). https://dl.acm.org/doi/book/10.5555/3279302 (accessed August 1, 2020).

[323] I. Ahmad, M. Basheri, M.J. Iqbal, A. Rahim, Performance Comparison of Support Vector Machine, Random Forest, and Extreme Learning Machine for Intrusion Detection, IEEE Access. 6 (2018) 33789-33795. https://doi.org/10.1109/ACCESS.2018.2841987.

[324] A. Datta, M.J. Augustin, N. Gupta, S.R. Viswamurthy, K.M. Gaddikeri, R. Sundaram, Impact Localization and Severity Estimation on Composite Structure Using Fiber Bragg Grating Sensors by Least Square Support Vector Regression, IEEE Sensors Journal. 19 (2019) 4463-4470. https://doi.org/10.1109/JSEN.2019.2901453.

[325] J. Zhang, Y. Wang, Evaluating the bond strength of FRP-to-concrete composite joints using metaheuristic-optimized least-squares support vector regression, Neural Comput \& Applic. (2020). https://doi.org/10.1007/s00521-020-05191-0.

[326] Z. Yang, X.S. Gu, X.Y. Liang, L.C. Ling, Genetic algorithm-least squares support vector regression based predicting and optimizing model on carbon fiber composite integrated conductivity, Materials \& Design. 31 (2010) 1042-1049. https://doi.org/10.1016/j.matdes.2009.09.057.

[327] A. Mardanshahi, V. Nasir, S. Kazemirad, M.M. Shokrieh, Detection and classification of matrix cracking in laminated composites using guided wave propagation and artificial neural networks, Composite Structures. 246 (2020) 112403. https://doi.org/10.1016/j.compstruct.2020.112403.

[328] M. Meng, Y.J. Chua, E. Wouterson, C.P.K. Ong, Ultrasonic signal classification and imaging system for composite materials via deep convolutional neural networks, Neurocomputing. 257 (2017) 128-135. https://doi.org/10.1016/j.neucom.2016.11.066.

[329] (17) (PDF) Classification of damages in composite images using Zernike moments and support vector machines, (n.d.).

https://www.researchgate.net/publication/329710067_Classification_of_damages_in_composite_image s_using_Zernike_moments_and_support_vector_machines (accessed August 1, 2020). 
[330] O. Abuomar, S. Nouranian, R. King, T.M. Ricks, T.E. Lacy, Comprehensive mechanical property classification of vapor-grown carbon nanofiber/vinyl ester nanocomposites using support vector machines, Computational Materials Science. 99 (2015) 316-325. https://doi.org/10.1016/j.commatsci.2014.12.029.

[331] U. Aich, R.R. Behera, S. Banerjee, Modeling of delamination in drilling of glass fiberreinforced polyester composite by support vector machine tuned by particle swarm optimization, Int $\mathbf{J}$ Plast Technol. 23 (2019) 77-91. https://doi.org/10.1007/s12588-019-09233-8.

[332] A particle swarm optimization-support vector machine hybrid system with acoustic emission on damage degree judgment of carbon fiber reinforced polymer cables - Jie Xu, Xuan Liu, Qinghua Han, Weixin Wang, 2020, (n.d.). https://journals.sagepub.com/doi/full/10.1177/1475921720922824?casa_token=478rHI6vokIAAAAA \%3AaogtmJiStpoFZNzhHSLwLbPRpDydsLzfANBfan3U9e61wetZoOdMW6XkJtZxchmltg25pbMp_ Px4J6T (accessed August 1, 2020).

[333] E. Koumoulos, G. Konstantopoulos, C. Charitidis, Applying Machine Learning to Nanoindentation Data of (Nano-) Enhanced Composites, Fibers. 8 (2020) 3. https://doi.org/10.3390/fib8010003.

[334] S. Altarazi, R. Allaf, F. Alhindawi, Machine Learning Models for Predicting and Classifying the Tensile Strength of Polymeric Films Fabricated via Different Production Processes, Materials. 12 (2019) 1475. https://doi.org/10.3390/ma12091475.

[335] S. Dey, T. Mukhopadhyay, S.K. Sahu, S. Adhikari, Effect of cutout on stochastic natural frequency of composite curved panels, Composites Part B: Engineering. 105 (2016) 188-202. https://doi.org/10.1016/j.compositesb.2016.08.028.

[336] Amandeep singh K, T.V. Anantham, S. Sathya, C.Tamilselvi, T. Mualidharan, Identification of project risk factor using support vector machine, International Journal of Advanced Science and Technology. 29 (n.d.) 2932-2938.

[337] V.U.B. Challagulla, F.B. Bastani, I.-L. Yen, R.A. Paul, Empirical assessment of machine learning based software defect prediction techniques, Int. J. Artif. Intell. Tools. 17 (2008) 389-400. https://doi.org/10.1142/S0218213008003947.

[338] D. Tien Bui, T.A. Tuan, H. Klempe, B. Pradhan, I. Revhaug, Spatial prediction models for shallow landslide hazards: a comparative assessment of the efficacy of support vector machines, artificial neural networks, kernel logistic regression, and logistic model tree, Landslides. 13 (2016) 361-378. https://doi.org/10.1007/s10346-015-0557-6.

[339] S. Das, A. Chattopadhyay, A.N. Srivastava, Classifying Induced Damage in Composite Plates Using One-Class Support Vector Machines, AIAA Journal. 48 (2010) 705-718. https://doi.org/10.2514/1.37282.

[340] Support vector machines in structural engineering: a review: Journal of Civil Engineering and $\begin{array}{llllll}\text { Management: } & \text { Vol } & 21, & \text { No } & 3, & \text { (n.d.). }\end{array}$ https://www.tandfonline.com/doi/abs/10.3846/13923730.2015.1005021 (accessed August 2, 2020).

[341] L. Rokach, Ensemble Methods for Classifiers, in: O. Maimon, L. Rokach (Eds.), Data Mining and Knowledge Discovery Handbook, Springer US, Boston, MA, 2005: pp. 957-980. https://doi.org/10.1007/0-387-25465-X_45.

[342] L. Rokach, Ensemble-based classifiers, Artif Intell Rev. 33 (2010) 1-39. https://doi.org/10.1007/s10462-009-9124-7.

[343] H. Beyer, Tukey, John W.: Exploratory Data Analysis. Addison-Wesley Publishing Company Reading, Mass. - Menlo Park, Cal., London, Amsterdam, Don Mills, Ontario, Sydney 1977, XVI, 688 S., Biometrical Journal. 23 (1981) 413-414. https://doi.org/10.1002/bimj.4710230408. 
[344] B.V. Dasarathy, B.V. Sheela, A composite classifier system design: Concepts and methodology, Proceedings of the IEEE. 67 (1979) 708-713. https://doi.org/10.1109/PROC.1979.11321.

[345] A.I. Weinberg, M. Last, Selecting a representative decision tree from an ensemble of decisiontree models for fast big data classification, Journal of Big Data. 6 (2019) 23. https://doi.org/10.1186/s40537-019-0186-3.

[346] D. Opitz, R. Maclin, Popular Ensemble Methods: An Empirical Study, Journal of Artificial Intelligence Research. 11 (1999) 169-198. https://doi.org/10.1613/jair.614.

[347] R. Liu, Y.C. Yabansu, A. Agrawal, S.R. Kalidindi, A.N. Choudhary, Machine learning approaches for elastic localization linkages in high-contrast composite materials, Integrating Materials. 4 (2015) 192-208. https://doi.org/10.1186/s40192-015-0042-z.

[348] Z. Zhang, J. Shi, T. Yu, A. Santomauro, A. Gordon, J. Gou, D. Wu, Predicting Flexural Strength of Additively Manufactured Continuous Carbon Fiber-Reinforced Polymer Composites Using Machine Learning, J. Comput. Inf. Sci. Eng. 20 (2020). https://doi.org/10.1115/1.4047477.

[349] Spatial-Neighborhood Manifold Learning for Nondestructive Testing of Defects in Polymer Composites - $\quad$ IEEE Journals $\quad$ \& $\quad$ Magazine, https://ieeexplore.ieee.org/abstract/document/8882239?casa_token=6IiaJhpHQAsAAAAA:i3VweUiV5 wFf5XQKjQlflcVCoDUVujpt8nE5xoscp8Y2dm-cIqp12Iv-ziuFv51PSRaFLB1_cdrCIA (accessed August 2, 2020).

[350] P. Gaudenzi, D. Nardi, I. Chiappetta, S. Atek, L. Lampani, F. Sarasini, J. Tirillo, T. Valente, impact damage detection in composite laminate plates using an integrated piezoelectric sensor and actuator couple combined with wavelet-based features extraction approach, (2015). /paper/impactdamage-detection-in-composite-laminate-using-Gaudenzi-

Nardi/b087a25fbfddd278b52f3d5f1ff5f0956577e7f1 (accessed August 2, 2020).

[351] A. Khan, N. Kim, J.K. Shin, H.S. Kim, B.D. Youn, Damage assessment of smart composite structures via machine learning: a review, JMST Adv. 1 (2019) 107-124. https://doi.org/10.1007/s42791-019-0012-2.

[352] H. Zhang, C. Wang, M. Li, X. Ji, J. Zhang, B. Yang, Fluorescent Nanocrystal-Polymer Composites from Aqueous Nanocrystals: Methods without Ligand Exchange, Chem. Mater. 17 (2005) 4783-4788. https://doi.org/10.1021/cm0502601.

[353] Z. Wang, F. Chegdani, N. Yalamarti, B. Takabi, B. Tai, M. El Mansori, S. Bukkapatnam, Acoustic Emission Characterization of Natural Fiber Reinforced Plastic Composite Machining Using a Random Forest Machine Learning Model, J. Manuf. Sci. Eng. 142 (2020). https://doi.org/10.1115/1.4045945.

[354] M.V. Pathan, S.A. Ponnusami, J. Pathan, R. Pitisongsawat, B. Erice, N. Petrinic, V.L. Tagarielli, Predictions of the mechanical properties of unidirectional fibre composites by supervised machine learning, Scientific Reports. 9 (2019) 13964. https://doi.org/10.1038/s41598-019-50144-w.

[355] H. Guo, J. Yin, J. Zhao, L. Yao, X. Xia, H. Luo, An Ensemble Learning for Predicting Breakdown Field Strength of Polyimide Nanocomposite Films, Journal of Nanomaterials. 2015 (2015) e950943. https://doi.org/10.1155/2015/950943.

[356] X. Dong, Z. Yu, W. Cao, Y. Shi, Q. Ma, A survey on ensemble learning, Front. Comput. Sci. 14 (2020) 241-258. https://doi.org/10.1007/s11704-019-8208-z.

[357] A.P. Bradley, The use of the area under the ROC curve in the evaluation of machine learning algorithms, Pattern Recognition. 30 (1997) 1145-1159. 
[358] M. Wieland, M. Pittore, Performance Evaluation of Machine Learning Algorithms for Urban Pattern Recognition from Multi-spectral Satellite Images, Remote Sensing. 6 (2014) 2912-2939. https://doi.org/10.3390/rs6042912.

[359] X. Deng, Q. Liu, Y. Deng, S. Mahadevan, An improved method to construct basic probability assignment based on the confusion matrix for classification problem, Information Sciences. 340-341 (2016) 250-261. https://doi.org/10.1016/j.ins.2016.01.033.

[360] J. Ling, J. Templeton, Evaluation of machine learning algorithms for prediction of regions of high Reynolds averaged Navier Stokes uncertainty, Physics of Fluids. 27 (2015) 085103. https://doi.org/10.1063/1.4927765.

[361] B.T. Pham, A. Jaafari, M. Avand, N. Al-Ansari, T. Dinh Du, H.P.H. Yen, T.V. Phong, D.H. Nguyen, H.V. Le, D. Mafi-Gholami, I. Prakash, H. Thi Thuy, T.T. Tuyen, Performance Evaluation of Machine Learning Methods for Forest Fire Modeling and Prediction, Symmetry. 12 (2020) 1022. https://doi.org/10.3390/sym12061022.

[362] A.K. Dwivedi, Performance evaluation of different machine learning techniques for prediction of heart disease, Neural Comput \& Applic. 29 (2018) 685-693. https://doi.org/10.1007/s00521-0162604-1.

[363] S. Garcia, A. Fernandez, J. Luengo, F. Herrera, A study of statistical techniques and performance measures for genetics-based machine learning: accuracy and interpretability, Soft Comput. 13 (2020) 959-977. https://doi.org/10.1007/s00500-008-0392-y.

[364] J. Li, J.-B. Martens, J.J. van Wijk, Judging Correlation from Scatterplots and Parallel Coordinate Plots, Information Visualization. 9 (2010) 13-30. https://doi.org/10.1057/ivs.2008.13.

[365] N.K. Ostadi, Comparison of Machine Learning Techniques for Developing Performance Prediction Models, Computing in civil and building engineering. (2014). https://doi.org/10.1061/9780784413616.152.

[366] R. Pandey, N. Srivastava, S. Fatima, Extending R Boxplot Analysis to Big Data in Education, in: 2015 Fifth International Conference on Communication Systems and Network Technologies, 2015: pp. 1030-1033. https://doi.org/10.1109/CSNT.2015.73.

[367] M. Krzywinski, N. Altman, Visualizing samples with box plots, Nature Methods. 11 (2014) 119-120. https://doi.org/10.1038/nmeth.2813.

[368] A. Li, M. Feng, Y. Li, Z. Liu, Application of outlier mining in insider identification based on Boxplot method, Procedia Computer Science. 91 (n.d.) 245-251.

[369] B. Efron, R. Tibshirani, Bootstrap Methods for Standard Errors, Confidence Intervals, and Other Measures of Statistical Accuracy, Statistical Science. 1 (1986) 54-75.

[370] C.E. Brodley, U. Rebbapragada, K. Small, B. Wallace, Challenges and Opportunities in Applied Machine Learning, AI Magazine. 33 (2012) 11-24. https://doi.org/10.1609/aimag.v33i1.2367.

[371] Y. Liu, T. Zhao, W. Ju, S. Shi, Materials discovery and design using machine learning, Journal of Materiomics. 3 (2017) 159-177. https://doi.org/10.1016/j.jmat.2017.08.002.

[372] M. Cacciola, S. Calcagno, F.C. Morabito, M. Versaci, Computational intelligence aspects for defect classification in aeronautic composites by using ultrasonic pulses, IEEE Transactions on Ultrasonics, Ferroelectrics, and Frequency Control. 55 (2008) 870-878. https://doi.org/10.1109/TUFFC.2008.722.

[373] D. Xu, P.F. Liu, Z.P. Chen, J.X. Leng, L. Jiao, Achieving robust damage mode identification of adhesive composite joints for wind turbine blade using acoustic emission and machine learning, Composite Structures. 236 (2020) 111840. https://doi.org/10.1016/j.compstruct.2019.111840. 
[374] Z. Zhang, K. Friedrich, Artificial neural networks applied to polymer composites: a review, Composites Science and Technology. 63 (2003) 2029-2044. https://doi.org/10.1016/S02663538(03)00106-4.

[375] P. Domingos, A few useful things to know about machine learning, Commun. ACM. 55 (2012) 78-87. https://doi.org/10.1145/2347736.2347755.

[376] Overfitting and undercomputing in machine learning | ACM Computing Surveys, (n.d.). https://dl.acm.org/doi/abs/10.1145/212094.212114?casa_token=nD8yjJdwYtsAAAAA\%3AwPlc6Tsiy zQVDn180qM5St7z6_3_iJCF_UEVea_nH-MMRn3PUhvVxSxviP8SXsa5OMm1_UqaclfPSA (accessed August 5, 2020).

[377] N. Mehrabi, F. Morstatter, N. Saxena, K. Lerman, A. Galstyan, A Survey on Bias and Fairness in Machine Learning, ArXiv:1908.09635 [Cs]. (2019). http://arxiv.org/abs/1908.09635 (accessed August 5, 2020).

[378] D. Koller, M. Sahami, Toward Optimal Feature Selection, (1996). http://ilpubs.stanford.edu:8090/208/ (accessed August 5, 2020).

[379] J. Cai, J. Luo, S. Wang, S. Yang, Feature selection in machine learning: A new perspective, Neurocomputing. 300 (2018) 70-79. https://doi.org/10.1016/j.neucom.2017.11.077.

[380] A.L. Blum, P. Langley, Selection of relevant features and examples in machine learning, Artificial Intelligence. 97 (1997) 245-271. https://doi.org/10.1016/S0004-3702(97)00063-5.

[381] M.A. Hall, Correlation-based Feature Selection for Machine Learning, 1999.

[382] C. Lee, G.G. Lee, Information gain and divergence-based feature selection for machine learning-based text categorization, Information Processing \& Management. 42 (2006) 155-165. https://doi.org/10.1016/j.ipm.2004.08.006.

[383] Lei Yu, Huan Liu, Feature Selection for High-Dimensional Data: A Fast Correlation-Based Filter Solution, Proceedings of the Twentieth International Conference on Machine Learning (ICML2003), Washington DC, 2003. (n.d.).

[384] A survey of feature selection and feature extraction techniques in machine learning - IEEE Conference Publication, (n.d.). https://ieeexplore.ieee.org/abstract/document/6918213 (accessed August 5, 2020).

[385] T.N. Lal, O. Chapelle, J. Weston, A. Elisseeff, Embedded Methods, in: I. Guyon, M. Nikravesh, S. Gunn, L.A. Zadeh (Eds.), Feature Extraction: Foundations and Applications, Springer, Berlin, Heidelberg, 2006: pp. 137-165. https://doi.org/10.1007/978-3-540-35488-8_6.

[386] Embedded feature selection for multi-label learning-- 《Journal of Nanjing University(Natural Sciences)》2009年05期， (n.d.). http://en.cnki.com.cn/Article_en/CJFDTotal-NJDZ200905013.htm (accessed August 5, 2020).

[387] D.R. Wilson, T.R. Martinez, Improved Heterogeneous Distance Functions, Journal of Artificial Intelligence Research. 6 (1997) 1-34. https://doi.org/10.1613/jair.346.

[388] B. McCane, M. Albert, Distance functions for categorical and mixed variables, Pattern Recognition Letters. 29 (2008) 986-993. https://doi.org/10.1016/j.patrec.2008.01.021.

[389] S.H. Kim, F. Boukouvala, Surrogate-based optimization for mixed-integer nonlinear problems, $\begin{array}{llllll}\text { Computers } \& \quad \text { Chemical } & \text { Engineering. } & 140 & \text { (2020) } & 106847 .\end{array}$ https://doi.org/10.1016/j.compchemeng.2020.106847.

[390] G. Nannicini, On the implementation of a global optimization method for mixed-variable problems, (2020). 
[391] F. Wang, H. Zhang, A. Zhou, A particle swarm optimization algorithm for mixed-variable optimization problems, Swarm and Evolutionary Computation. $60 \quad$ (2021) 100808. https://doi.org/10.1016/j.swevo.2020.100808.

[392] M. Egmont-Petersen, J.L. Talmon, A. Hasman, Robustness metrics for measuring the influence of additive noise on the performance of statistical classifiers, International Journal of Medical Informatics. 46 (1997) 103-112. https://doi.org/10.1016/S1386-5056(97)00062-2.

[393] Robust Statistics - Peter J. Huber - Google Books, (n.d.). https://books.google.co.in/books?hl=en\&lr=\&id=e62RhdqIdMkC\&oi=fnd\&pg=PA1\&ots=fA0186MhL $\mathrm{g} \&$ sig=zo9ib0mZoEI7ARZjKf-Zlm620RQ\&redir_esc=y\#v=onepage\&q\&f=false (accessed December 2, 2020).

[394] J.A. Sáez, J. Luengo, F. Herrera, Evaluating the classifier behavior with noisy data considering performance and robustness: The Equalized Loss of Accuracy measure, Neurocomputing. 176 (2016) 26-35. https://doi.org/10.1016/j.neucom.2014.11.086.

[395] Y. Kharin, E. Zhuk, Robustness in statistical pattern recognition under "contaminations" of training samples, in: Proceedings of the 12th IAPR International Conference on Pattern Recognition, Vol. 3 - Conference C: Signal Processing (Cat. No.94CH3440-5), 1994: pp. 504-506 vol.2. https://doi.org/10.1109/ICPR.1994.576996.

[396] J.A. Sáez, J. Luengo, F. Herrera, Fuzzy Rule Based Classification Systems versus crisp robust learners trained in presence of class noise's effects: A case of study, 2011 11th International Conference on Intelligent Systems Design and Applications. (2011). https://doi.org/10.1109/ISDA.2011.6121827.

[397] E. Kalapanidas, N. Avouris, M. Craciun, D. Neagu, Machine Learning algorithms: a study on noise sensitivity, (2003).

[398] T. Elberfeld, J. De Beenhouwer, A.J. den Dekker, C. Heinzl, J. Sijbers, Parametric Reconstruction of Glass Fiber-reinforced Polymer Composites from X-ray Projection Data-A Simulation Study, J Nondestruct Eval. 37 (2018) 62. https://doi.org/10.1007/s10921-018-0514-0.

[399] Z. Yan, C.-Y. Chen, L. Luo, Y. Yao, Stable principal component pursuit-based thermographic data analysis for defect detection in polymer composites, Journal of Process Control. 49 (2017) 36-44. https://doi.org/10.1016/j.jprocont.2016.11.008.

[400] K. Zheng, Y.-S. Chang, K.-H. Wang, Y. Yao, Improved non-destructive testing of carbon fiber reinforced polymer (CFRP) composites using pulsed thermograph, Polymer Testing. 46 (2015) 26-32. https://doi.org/10.1016/j.polymertesting.2015.06.016.

[401] T. Mukhopadhyay, T.K. Dey, R. Chowdhury, A. Chakrabarti, Structural Damage Identification Using Response Surface-Based Multi-objective Optimization: A Comparative Study, Arab J Sci Eng. 40 (2015) 1027-1044. https://doi.org/10.1007/s13369-015-1591-3.

[402] S. Dey, S. Naskar, T. Mukhopadhyay, U. Gohs, A. Spickenheuer, L. Bittrich, S. Sriramula, S. Adhikari, G. Heinrich, Uncertain natural frequency analysis of composite plates including effect of noise - A polynomial neural network approach, Compos Struct. 143 (2016) 130-142. https://doi.org/10.1016/j.compstruct.2016.02.007.

[403] T. Mukhopadhyay, S. Naskar, S. Dey, S. Adhikari, On quantifying the effect of noise in surrogate based stochastic free vibration analysis of laminated composite shallow shells, Compos Struct. 140 (2016). https://doi.org/10.1016/j.compstruct.2015.12.037.

[404] A.T. Saseendran, L. Setia, V. Chabbria, D. Chakraborty, A.B. Roy, Impact of Noise in Dataset on Machine Learning Algorithms, (n.d.). 
[405] S. Gupta, A. Gupta, Dealing with Noise Problem in Machine Learning Data-sets: A Systematic Review, Procedia Computer Science. 161 (2019) 466-474. https://doi.org/10.1016/j.procs.2019.11.146. [406] S. Verbaeten, A. Van Assche, Ensemble Methods for Noise Elimination in Classification Problems, in: T. Windeatt, F. Roli (Eds.), Multiple Classifier Systems, Springer Berlin Heidelberg, Berlin, Heidelberg, 2003: pp. 317-325. https://doi.org/10.1007/3-540-44938-8_32.

[407] B. Sluban, D. Gamberger, N. Lavra, Advances in Class Noise Detection, in: Proceedings of the 2010 Conference on ECAI 2010: 19th European Conference on Artificial Intelligence, IOS Press, NLD, 2010: pp. 1105-1106.

[408] D.W. Aha, D. Kibler, M.K. Albert, Instance-based learning algorithms, Mach Learn. 6 (1991) 37-66. https://doi.org/10.1007/BF00153759.

[409] X. Zhao, M. Li, G. Song, J. Xu, Hierarchical ensemble-based data fusion for structural health monitoring, Smart Mater. Struct. 19 (2010) 045009. https://doi.org/10.1088/0964-1726/19/4/045009.

[410] J.-R. Yeh, J.-S. Shieh, N.E. Huang, Complementary ensemble empirical mode decomposition: a novel noise enhanced data analysis method, Adv. Adapt. Data Anal. 02 (2010) 135-156. https://doi.org/10.1142/S1793536910000422.

[411] M. Abdelrahim, C. Merlos, T.( Wang, Hybrid Machine Learning Approaches: A Method to Improve Expected Output of Semi-structured Sequential Data, in: 2016 IEEE Tenth International Conference on Semantic Computing (ICSC), 2016: pp. 342-345. https://doi.org/10.1109/ICSC.2016.72.

[412] T. Shon, J. Moon, A hybrid machine learning approach to network anomaly detection, Information Sciences. 177 (2007) 3799-3821. https://doi.org/10.1016/j.ins.2007.03.025.

[413] Y. Sun, G. Li, J. Zhang, Developing Hybrid Machine Learning Models for Estimating the Unconfined Compressive Strength of Jet Grouting Composite: A Comparative Study, Applied Sciences. 10 (2020) 1612. https://doi.org/10.3390/app10051612.

[414] C. Qi, H.-B. Ly, Q. Chen, T.-T. Le, V.M. Le, B.T. Pham, Flocculation-dewatering prediction of fine mineral tailings using a hybrid machine learning approach, Chemosphere. 244 (2020) 125450. https://doi.org/10.1016/j.chemosphere.2019.125450.

[415] T.-T. Le, Practical Hybrid Machine Learning Approach for Estimation of Ultimate Load of Elliptical Concrete-Filled Steel Tubular Columns under Axial Loading, Advances in Civil Engineering. 2020 (2020) e8832522. https://doi.org/10.1155/2020/8832522.

[416] (PDF) Stochastic Oblique Impact on Composite Laminates: A Concise Review and Characterization of the Essence of Hybrid Machine Learning Algorithms, ResearchGate. (n.d.). https://doi.org/10.1007/s11831-020-09438-w.

[417] D.-T. Vu, N.-D. Hoang, Punching shear capacity estimation of FRP-reinforced concrete slabs using a hybrid machine learning approach, Structure and Infrastructure Engineering. 12 (2016) 11531161. https://doi.org/10.1080/15732479.2015.1086386.

[418] Z. Wu, D. Rincon, P.D. Christofides, Real-Time Adaptive Machine-Learning-Based Predictive Control of Nonlinear Processes, Ind. Eng. Chem. Res. 59 (2020) 2275-2290. https://doi.org/10.1021/acs.iecr.9b03055.

[419] S. Yang, M. Cho, Scale bridging method to characterize mechanical properties of nanoparticle/polymer nanocomposites, Appl. Phys. Lett. $93 \quad$ (2008) 043111. https://doi.org/10.1063/1.2965486.

[420] J. Knap, C. Spear, K. Leiter, R. Becker, D. Powell, A computational framework for scalebridging in multi-scale simulations: A computational framework for scale-bridging in multi-scale 
simulations, International Journal for Numerical Methods in Engineering. 108 (2016). https://doi.org/10.1002/nme.5270.

[421] N. Lubbers, A. Agarwal, Y. Chen, S. Son, M. Mehana, Q. Kang, S. Karra, C. Junghans, T.C. Germann, H.S. Viswanathan, Modeling and scale-bridging using machine learning: nanoconfinement effects in porous media, Scientific Reports. 10 (2020) 13312. https://doi.org/10.1038/s41598-02069661-0.

[422] K.W. Leiter, B.C. Barnes, R. Becker, J. Knap, Accelerated scale-bridging through adaptive surrogate model evaluation, Journal of Computational Science. 27 (2018) 91-106. https://doi.org/10.1016/j.jocs.2018.04.010.

[423] J. Fish, Bridging the scales in nano engineering and science, J Nanopart Res. 8 (2006) 577-594. https://doi.org/10.1007/s11051-006-9090-9.

[424] A. Aruna, R. Ganguli, Multi-fidelity response surfaces for uncertainty quantification in beams using coarse and fine finite element discretizations, International Journal for Computational Methods in $\begin{array}{llllll}\text { Engineering } & \text { Science } & \text { and } & \text { Mechanics. } & \text { (2021) }\end{array}$ https://doi.org/10.1080/15502287.2020.1849447.

[425] K. V. Vishal Krishnan, R. Ganguli, Multi-fidelity analysis and uncertainty quantification of beam vibration using co-kriging interpolation method, Applied Mathematics and Computation, 398 (2021) 125987. https://doi.org/10.1016/j.amc.2021.125987 